\title{
A Distributed Framework with a Novel Pricing Model for Dynamic Spectrum Access to Secondary Users in Infrastructure based Wireless Networks
}

\author{
by
}

\section{Soumitra Dixit}

\author{
A thesis submitted to the \\ Faculty of Graduate Studies and Research \\ in partial fulfillment of the requirements for the degree of
}

Master of Applied Science in Electrical and Computer Engineering

Ottawa-Carleton Institute for Electrical and Computer Engineering (OCIECE) Department of Systems and Computer Engineering

Carleton University

Ottawa, Ontario, Canada

January 2010

(C) Soumitra Dixit, 2010 
Library and Archives

Canada

Published Heritage

Branch

395 Wellington Street

Ottawa ON K1A ON4

Canada
Bibliotheque et

Archives Canada

Direction du

Patrimoine de l'édition

395 , rue Wellington

Ottawa ON K1A ON4

Canada
Your file Votre référence
ISBN: $978-0-494-63822-4$
Our file Notre référence
ISBN: $978-0-494-63822-4$

\section{NOTICE:}

The author has granted a nonexclusive license allowing Library and Archives Canada to reproduce, publish, archive, preserve, conserve, communicate to the public by telecommunication or on the Internet, loan, distribute and sell theses worldwide, for commercial or noncommercial purposes, in microform, paper, electronic and/or any other formats.

The author retains copyright ownership and moral rights in this thesis. Neither the thesis nor substantial extracts from it may be printed or otherwise reproduced without the author's permission.

\section{AVIS:}

L'auteur a accordé une licence non exclusive permettant à la Bibliothèque et Archives Canada de reproduire, publier, archiver, sauvegarder, conserver, transmettre au public par télécommunication ou par l'Internet, prêter, distribuer et vendre des thèses partout dans le monde, à des fins commerciales ou autres, sur support microforme, papier, électronique et/ou autres formats.

L'auteur conserve la propriété du droit d'auteur et des droits moraux qui protège cette thèse. $\mathrm{Ni}$ la thèse ni des extraits substantiels de celle-ci ne doivent être imprimés ou autrement reproduits sans son autorisation.
In compliance with the Canadian Privacy Act some supporting forms may have been removed from this thesis.

While these forms may be included in the document page count, their removal does not represent any loss of content from the thesis.
Conformément à la loi canadienne sur la protection de la vie privée, quelques formulaires secondaires ont été enlevés de cette thèse.

Bien que ces formulaires aient inclus dans la pagination, il n'y aura aucun contenu manquant.

\section{Canadä}




\begin{abstract}
\end{abstract}
Ever increasing demand for radio spectrum and its current underutilization have made the radio spectrum a scarce and expensive resource. In this thesis, a system for allowing unsubscribed Secondary User (SU) access to the underutilized spectrum in the licensed bands in infrastructure based networks is proposed, with minimum modifications to the current Wireless Service Provider (WSP) infrastructure. The proposed distributed system framework enables the orthogonal co-existence of subscribed Primary Users (PUs) and SUs at the same Base Station (BS).

The rationale of this thesis is the paradoxical fact that, the WSPs invest heavily in licensing the spectrum, and this licensed spectrum goes underutilized due to the variable usage in time and location of the subscribed PUs. Allowing temporary access to SUs at the BSs of the WSPs, whenever the spectrum is underutilized, presents an opportunity for the WSPs to generate additional profits from SU access.

The aim of this thesis is to present a system framework for enabling $\mathrm{SU}$ access at the BSs considering both the technological and the economic perspectives. The signaling framework for SU access, SU identification, PU-SU joint resource management model, autonomous BS selection and a price-based handoff at the SU terminal are the key technological contributions in this thesis. The dynamic incentive based pricing model for charging SUs, along with equilibrium analysis with differentiation of SU wireless service for realizing competitive SU pricing among multiple WSPs are the key economic contributions.

The proposed framework and pricing model is aimed at achieving the dual goal of temporary wireless access for unsubscribed SUs, as well as generating additional profits to the WSPs. This proposed framework is thus an intermediate step between the current infrastructure based networks and the cognitive networks of the future. 


\section{Acknowledgments}

I am extremely grateful to my supervisors Prof. Shalini Periyalwar and Prof. Halim Yanikomeroglu for their guidance, support, generosity, patience and encouragement during the course of my research. The valuable discussions with you have always been very enlightening for me, and have helped me immensely in developing the ideas presented in this thesis. I also thank you for providing me with the ideal research environment and the inspirational group meetings making my research a very enjoyable experience. It has been an absolute pleasure to have worked with you over the past two years!

I am also extremely thankful to Dr. Petar Djukic, Akram Salem Bin Sediq, Mahmudur Rahman, Mohamed Rashad, Ghassan Dahman, Muhammad Al-Juaid, Saad El-Ahmadi, Hussein Zubaidy, Sebastian Szyszkowicz, Furuzan Atay Onat, Feroz Bokhari, Dr. Abdulkareem Adinoyi, Stefan Valentin, Tarik Shehata, Alireza Sharifian, Talha Ahmad, Heba Eid, Emil Janulewicz, and Bo Liu for the fruitful discussions, encouragement and extremely useful feedback during the past two years.

I also would like to express my gratitude to my dear friends Chandan Mohapatra and Subra Mohapatra and my landlords Mr. and Mrs. Nagy, who have been very helpful and supportive throughout the course of the past two years.

I am also very thankful to the SCE department office staff for their help and co-ordination, as well as the technical staff at the SCE department in providing me with all the necessary softwares and solving my computer related problems.

Finally, I thank my parents and my little brother Sanat for their constant support, encouragement and patience throughout the course of my masters degree. 


\section{Table of Contents}

$\begin{array}{ll}\text { Abstract } & \text { ii }\end{array}$

$\begin{array}{ll}\text { Acknowledgments } & \text { iii }\end{array}$

Table of Contents $\quad$ iv

List of Tables $\quad$ vii

List of Figures viii

List of Acronyms $\quad$ x

List of Symbols $\quad$ xiii

Chapter 1: Introduction $\quad \mathbf{1}$

1.1 Dynamic Spectrum Access Techniques . . . . . . . . . . . . . 2

1.1.1 Cognitive Radio and Current Mobile Device Trends . . . . . . 3

1.1.2 Classification of DSA Techniques ........... 4

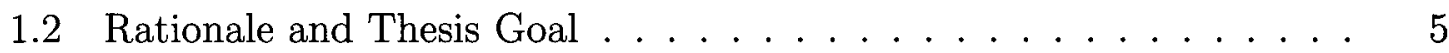

1.2.1 Proposed Distributed System Framework . . . . . . . . . 6

1.2.2 Thesis Structure ....................... 8

1.2.3 Scope of the Thesis . . . . . . . . . . . . 10

1.3 Thesis Contributions ................... . . 11

1.4 Organization of the Thesis . . . . . . . . . . . . . . 13

1.5 Manuscripts Published, Submitted and In-progress . . . . . . . . . . 14

Chapter 2: $\quad$ System Framework 16

2.1 Literature Review . . . . . . . . . . . . . . . . . 16

2.1.1 Classification of Licensed Band Spectrum Sharing Techniques 16

2.1.2 Centralized Architectures . . . . . . . . . . . . 17

2.1.3 Distributed Architectures ............. . . 20

2.2 Motivation for the Proposed Framework . . . . . . . . . . . . 21

2.2 .1 Key Features . . . . . . . . . . . . . . . . 22

2.3 System Model . . . . . . . . . . . . . . . . . . . . . . . . . . . . . . . . . 23

2.3.1 Enhancements for Enabling SU Access . . . . . . . . . . 24

2.3 .2 SU Terminal . . . . . . . . . . . . . . . . . . 25

2.4 Signaling Framework for SU - BS Interaction . . . . . . . . . . . 27

2.4 .1 Initialization . . . . . . . . . . . . . . 27 
2.4 .2 Authentication . . . . . . . . . . . . . . . . 29

2.4.3 Connection Establishment . . . . . . . . . . . . . 30

2.4.4 Modified Signaling Framework for SU Type B . . . . . . . . 31

2.5 Identification of SUs using the DSCP Byte . . . . . . . . . . . 33

2.6 Structure for joint PU-SU RRM at the BS . . . . . . . . . . . 34

2.7 Summary . . . . . . . . . . . . . . . . . 37

Chapter 3: $\quad$ Network Selection and Price-based Handoff 38

3.1 Literature Review . . . . . . . . . . . . . . . . . . . . . . 39

3.2 Basic Criterion for Autonomous BS Selection at the SU Terminal . . 40

3.2.1 Rationale behind the Network Selection Criterion . . . . . . . 43

3.2.2 BS Selection Criterion for Single Application Class . . . . . . 44

3.3 Generalized BS Selection Criterion at the SU terminal . . . . . . . 46

3.4 Pricing based Handoff Initiated by the SU Terminal . . . . . . . . . 48

3.4.1 Motivation . . . . . . . . . . . . . . . . . 48

3.4 .2 Price-based Handoff Scheme . . . . . . . . . . . . 49

3.5 Summary . . . . . . . . . . . . . . . . 51

Chapter 4: Incentive based Pricing Model for Charging SUs $\quad 52$

4.1 Introduction . . . . . . . . . . . . . . . . 52

4.2 Literature Review . . . . . . . . . . . . . . . . . . . 54

4.3 Incentive based Pricing Model for Secondary Users . . . . . . . . 56

4.3.1 Parameters and Terminology . . . . . . . . . . . . . 57

4.3.2 Formulation for the Incentive based Pricing Model . . . . . . . 60

4.3.3 Pricing Flexibility with Price Leveling Factor . . . . . . . . 62

4.3.4 Dynamic Nature of the Pricing Model . . . . . . . . . . . 65

4.4 Summary . . . . . . . . . . . . . . . 66

Chapter 5: $\quad$ Game Theoretic Equilibrium Analysis for SU Pricing 68

5.1 Rationale . . . . . . . . . . . . . . . . . . 69

5.1 .1 Static Pricing v/s Dynamic Pricing . . . . . . . . . . . 69

5.1.2 Oligopoly and the Need for Equilibrium Analysis . . . . . 70

5.1 .3 Cooperation v/s Non-Cooperation . . . . . . . . . . . 73

5.1.4 Difficulties in Equilibrium Analysis with Dynamic Pricing . . 74

5.1.5 Strategy for Achieving Inter-WSP Competitive Pricing . . . . 75

5.2 Literature Review and Rationale . . . . . . . . . . . . 76

5.3 Oligopoly and SU Wireless Service Differentiation . . . . . . . . . 76

5.4 Game Theoretic Analysis for SU Pricing . . . . . . . . . . . . 78

5.4 .1 Linear City Model . . . . . . . . . . . . . . . 78

5.4 .2 Equilibrium Analysis for SU Pricing . . . . . . . . . . . 80

5.4.3 Implementation to the Dynamic Pricing Model . . . . . . . . . 84

5.5 Simulations and Results . . . . . . . . . . . . . . . 85

5.5.1 Channel Model for the Linear City . . . . . . . . . . . 85

5.5.2 Performance Results . . . . . . . . . . . . . . . . 86 
5.5.3 Effect of Assumptions on the WSP Profits . . . . . . . . 98

5.6 Summary . . . . . . . . . . . . . . . . . . . . 99

Chapter 6: Conclusions, Applications and Future Work 101

6.1 Conclusions . . . . . . . . . . . . . . . . . . . . . 101

6.1 .1 Technological Aspect . . . . . . . . . . . . . . . . 102

6.1 .2 Economic Aspect . . . . . . . . . . . . . . . . 102

6.2 Applications . . . . . . . . . . . . . . . . . . . . . 103

6.2.1 Prepaid Customers in Current Infrastructure based Networks . 103

6.2.2 Dynamic Pricing for Generalized Commercial Applications . . 103

6.3 Future Work . . . . . . . . . . . . . . . . . . . . . . . 104

$\begin{array}{ll}\text { References } & 105\end{array}$

$\begin{array}{lr}\text { Appendix A: Microeconomics } & 109\end{array}$

A.1 Demand Curve . . . . . . . . . . . . . . . . . . . . . . 109

A.2 Microeconomics Models ... . . . . . . . . . . . . . . 111

A.2.1 Forms of Economic Markets and Oligopoly . . . . . . . . 111

A.2.2 Imperfect Competition based on Prices . . . . . . . . . . 112

A.2.3 Differentiation of Products . . . . . . . . . . . . . . 114 


\section{List of Tables}

5.1 WSP duopoly: configuration parameters and WSP costs . . . . . 71

5.2 Channel model: simulation parameters . . . . . . . . . . 86

5.3 Scenario 1: WSP parameters and results . . . . . . . . . . 90

5.4 Scenario 2: WSP parameters and results . . . . . . . . . . . 92

5.5 Scenario 3: WSP parameters and results . . . . . . . . . . 94

5.6 Scenario 4: WSP parameters and results . . . . . . . . . . 96 


\section{List of Figures}

1.1 Spectrum measurements at peak hours on weekdays . . . . . . . 2

1.2 Snapshot of the spectrum band licensed by the WSP at the BS . . . 6

1.3 Technological and economic aspects - structure of the thesis $\ldots \ldots 9$

1.4 Thesis contributions $\ldots \ldots \ldots \ldots \ldots \ldots$

2.1 DIMSUMnet architecture . . . . . . . . . . . . . 18

2.2 Hybrid CPC access scheme . . . . . . . . . . . . . . 20

2.3 Network scenario with SU requiring wireless access $\ldots \ldots \ldots 24$

2.4 Signaling framework: initialization $\ldots \ldots \ldots \ldots \ldots$

2.5 Signaling framework: authentication $\ldots \ldots \ldots \ldots$

2.6 Signaling framework: connection establishment . . . . . . . . . . . 31

2.7 Signaling framework: SU type B . . . . . . . . . . . . . . . 32

2.8 DSCP byte and the proposed use of the rightmost bit for SUs . . . 34

2.9 Model for joint PU-SU RRM at the BS . . . . . . . . . . 35

3.1 SU terminal obtaining spectrum information from multiple BSs . . . 43

4.1 Demand curve and incentive based pricing . . . . . . . . . . 53

4.2 Pricing mechanism with spectrum policy server $\ldots \ldots \ldots 6$

4.3 Variable nature of the incentive based SU pricing model . . . . . . . . 61

4.4 Effect of different PLFs on the normalized SU price . . . . . . . 64 viii 
5.1 Pricing parameters: $\mathrm{WSP}_{1}$ and $\mathrm{WSP}_{2} \ldots \ldots \ldots \ldots$

5.2 Linear city model . . . . . . . . . . . . . . . . . 79

5.3 Spectral efficiencies over the linear city . . . . . . . . . 87

5.4 Scenario 1: SU price set by the two WSPs . . . . . . . . . 91

5.5 Scenario 1: CP obtained by the two WSPs from SU access . . . . . 91

5.6 Scenario $2:$ SU price set by the two WSPs $\ldots \ldots \ldots . \ldots 9$

5.7 Scenario 2: CP obtained by the two WSPs from SU access . . . . . 93

5.8 Scenario 3: SU price set by the two WSPs $\ldots \ldots \ldots \ldots$

5.9 Scenario 3: CP obtained by the two WSPs from SU access $\ldots \ldots .95$

5.10 Scenario 4: SU price set by the two WSPs $\ldots \ldots \ldots$. . . . . 97

5.11 Scenario 4: CP obtained by the two WSPs from SU access $\ldots \ldots .97$

A.1 Demand Curve $\ldots \ldots \ldots \ldots \ldots \ldots$ 


\section{List of Acronyms}

\begin{tabular}{ll}
\hline $2 \mathrm{G}$ & Second Generation \\
$3 \mathrm{G}$ & Third Generation \\
$4 \mathrm{G}$ & Fourth Generation \\
$\mathrm{AS}$ & Authentication Server \\
BER & Bit Error Rate \\
BR & Best Response \\
BS & Base Station \\
CAB & Coordinated Access Band \\
CAPEX & CAPital EXpenditure \\
CDMA & Code Division Multiple Access \\
CP & Cumulative Profit \\
CPC & Cognitive Pilot Channel \\
CME & Centralized Mediating Entity \\
CR & Cognitive Radio \\
CRRM & Common Radio Resource Management \\
DIMSUMnet & Dynamic Intelligent Management of Spectrum for Ubiq- \\
& uitous Mobile Networks \\
&
\end{tabular}




\begin{tabular}{ll} 
DSA & Dynamic Spectrum Access \\
DSCP & Differentiated Service Code Point \\
dB & Decibel \\
E2R & End-to-End Reconfigurability \\
EDGE & Enhanced Data rates for GSM Evolution \\
FP6 & Sixth Framework Programme \\
FDD & Frequency Division Duplexing \\
F-SUAkCH & Forward link Secondary User Acknowledgment Channel \\
F-SUBCH & Forward link Secondary User Broadcast Channel \\
F-SUCCH & Forward link Secondary User Control Channel \\
F-SUSCH & Forward link Secondary User Shared Channel \\
FTP & File Transfer Protocol \\
GSM & Global System for Mobile communications \\
ICF & Incentive Cutoff Factor \\
IETF & Internet Engineering Task Force \\
IP & Internet Protocol \\
LTE & Long Term Evolution \\
LTE-A & Long Term Evolution-Advanced \\
MAC & Medium Access Control \\
NE & Nash Equilibrium \\
NRT & Non Real Time \\
\hline
\end{tabular}




$\begin{array}{ll}\text { OFDM } & \text { Orthogonal Frequency Division Multiplexing } \\ \text { OPEX } & \text { OPerational EXpenditure } \\ \text { PLF } & \text { Price Leveling Factor } \\ \text { PU } & \text { Primary User } \\ \text { QoS } & \text { Quality of Service } \\ \text { RAT } & \text { Radio Access Technology } \\ \text { RNC } & \text { Radio Network Controller } \\ \text { RRM } & \text { Radio Resource Management } \\ \text { R-SUACH } & \text { Reverse link Secondary User Access Channel } \\ \text { R-SUCCH } & \text { Reverse link Secondary User Control Channel } \\ \text { R-SUSCH } & \text { Reverse link Secondary User Shared Channel } \\ \text { RT } & \text { Real Time } \\ \text { SMS } & \text { Short Messaging Service } \\ \text { SPS } & \text { Spectrum Policy Server } \\ \text { SNR } & \text { Signal to Noise Ratio } \\ \text { SU } & \text { Secondary User } \\ \text { SUF } & \text { Spectrum Utilization Factor } \\ \text { TDD } & \text { Time Division Duplexing } \\ \text { UMTS } & \text { Universal Mobile Telecommunications System } \\ \text { WSP } & \text { Wireless Service Provider } \\ \text { with respect to }\end{array}$




\section{List of Symbols}

\begin{abstract}
$\mathrm{BS}_{i}$, where $i \in\{1,2, \ldots, w\}$ The index for the BS considering that the SU is located in the coverage area of $w$ BSs.

$s_{i, j}$ The price charged by the $\mathrm{BS}_{i}$ to the SUs for temporary wireless access with application class $j$.

$p_{i, j}$ The fixed reference price charged by the $\mathrm{BS}_{i}$ to the PUs for guaranteed wireless access with application class $j$.

$c_{i, j}$ The cost incurred by the WSPs to provide a single SU with temporary wireless access for application class $j$ at $\mathrm{BS}_{i}$.

$\gamma_{i}$ The SNR detected by the SU on scanning the F-SUBCH transmitted from the $\mathrm{BS}_{i}$, such that $\gamma_{i}$ is a positive real number.

$\eta_{i}$ The average achievable link spectral efficiency based on the SNR $\gamma_{i}$ for the $\mathrm{SU}^{-\mathrm{BS}_{i}}$ link, where $\eta_{i}$ is also assumed to be a positive real number.
\end{abstract}


$A_{o, i}=\{j: j \in \mathbb{N}, j \leq m\} \quad$ The set of application classes offered by the $\mathrm{BS}_{i}$ to the SUs, where $j$ is the index for the application class and the maximum number of application classes offered by the BS of a WSP are assumed to be positive integer $m . \mathbb{N}$ indicates the set of natural numbers.

$A_{r}=\{j: j \in \mathbb{N}, j \leq n\} \quad$ The set of application classes requested by the SU for temporary wireless access, where the the maximum number of application classes that can be requested is limited by the positive integer $n$.

$B_{s l}$ The set of BSs, which provide the temporary wireless access for application classes requested by the $\mathrm{SU}\left(A_{r}\right)$ at the same minimum $\mathrm{SU}$ price.

$B E R_{j}$ Bit error rate for application class $j$.

$\mathcal{R}_{\mathcal{B}}$ The set of RATs $x$ supported by the $\mathrm{BS}_{i}$, where $x$ is the index of the RAT.

$\mathcal{R}_{\mathcal{S}}$ The set of RATs $x$ supported by the SU terminal. $I_{i, j}$ A binary integer variable for checking the availability of the $j^{\text {th }}$ application class requested by the SU with those offered by the $i^{\text {th }}$ BS. 
$B_{i, x} \quad$ A binary integer variable for verifying the support for RAT $x$ provided by $\mathrm{BS}_{i}$ at the $\mathrm{SU}$ terminal.

$M \quad$ A positive integer with a very large value.

$i^{*} \quad$ Index for the selected BS.

$C_{t h} \quad$ Cost threshold for triggering a price-based handoff; set by the SU terminal.

$C_{b d} \quad$ Cost boundary beyond which the SU cannot afford to continue with temporary wireless access.

$\alpha_{i, p u} \quad \mathrm{PU}$ utilization or PU demand at $\mathrm{BS}_{i} ; \alpha_{i, p u} \in[0,1]$.

$\alpha_{i, h} \quad$ Spectrum reserved for handoff and overload protection; $\alpha_{i, h} \in$ $[0,1]$

$\alpha_{i, t h} \quad$ Spectrum usage threshold at $\mathrm{BS}_{i} ; \alpha_{i, t h} \in[0,1]$.

$\alpha_{i, s u} \quad$ SU utilization at $\mathrm{BS}_{i} ; \alpha_{i, s u} \in[0,1]$.

$\alpha_{i, t} \quad$ Total utilization, i.e. SUF at $\mathrm{BS}_{i} ; \alpha_{i, t} \in[0,1]$.

$\alpha_{i, i c} \quad$ Incentive cutoff limit at $\mathrm{BS}_{i} ; \alpha_{i, i c} \in[0,1]$.

$f_{i, j}\left(\alpha_{i, t}\right)$ The normalized SU price with respect to PU price charged by the $\mathrm{BS}_{i}$ to the SUs for temporary wireless access with application class $j$.

$n_{i, j} \quad$ A positive real number representing the exponent in $f_{i}\left(\alpha_{i, t}\right)$ referred to as the ICF set at $\mathrm{BS}_{i}$ for application class $j$. 
$m_{i, j} \quad$ A non-negative real number representing the PLF at $\mathrm{BS}_{i}$ for the application class $j$.

$s_{c} \quad$ Positive real number for price adjustment for ensuring SU price is not equal to zero at zero SUF.

$T_{u} \quad$ Period of time in minutes in between the updating of the SU price by the $\mathrm{BS}_{i}$ based on PU demand.

$y \quad$ The unit distance of the linear city; $y \in[0,1]$.

$q \quad$ Number of SUs on the linear city.

$S_{i, j} \quad$ Static price paid by SUs on the linear city for application class $j$.

$C_{i, j} \quad$ Static cost borne by $\mathrm{WSP}_{i}$ per SU on the linear city for application class $j$.

$F_{i, j} \quad$ Fixed cost (CAPEX) per user to $\mathrm{WSP}_{i}$ for application class $j$.

$V_{i, j}\left(\alpha_{i, t}\right) \quad$ Variable cost (OPEX) per user to $\mathrm{WSP}_{i}$ for application class $j$.

$U_{i, j}(y) \quad$ Perceived price for temporary wireless access from BS of $\mathrm{WSP}_{i}$ to the SU located at any distance $y$ on the linear city for application class $j$.

$\zeta \quad$ Dissatisfaction price for the SUs on the linear city.

$D_{1}\left(S_{1}, S_{2}\right) \quad$ SU demand for WSP 1 on the linear city represented as a function of the $\mathrm{SU}$ prices set by $\mathrm{WSP}_{1}$ and $\mathrm{WSP}_{2}$.

$\pi_{1}\left(S_{1}, S_{2}\right) \quad$ Profit from SU access for $\mathrm{WSP}_{1}$ represented as a function of the $\mathrm{SU}$ prices set by $\mathrm{WSP}_{1}$ and $\mathrm{WSP}_{2}$. 
$S L_{i} \quad$ Satisfaction Level of a SU on the linear city.

$\sigma_{i} \quad$ Standard Deviation of the wireless channel on the linear city.

$S_{i, j}^{*} \quad \mathrm{NESU}$ price for $\mathrm{WSP}_{i}$ for application class $j$ on the linear city.

$B R_{1, j}\left(s_{2, j}\right) \quad$ Best response $\mathrm{SU}$ price for $\mathrm{WSP}_{1}$ with respect to the $\mathrm{SU}$ price set by $\mathrm{WSP}_{2}$.

$C P_{i, j} \quad$ Cumulative profit earned by $\mathrm{WSP}_{i}$ for application class $j$.

$\mathfrak{d} \quad$ Distance in meters used in the path loss model.

$P_{k} \quad$ Price set by the $k^{\text {th }}$ firm.

$Q_{k} \quad$ Quantity demanded or manufactured by the $k^{\text {th }}$ firm.

$P(M) \quad$ Price charged by the firm in a product monopoly.

$P(C) \quad$ Price charged by a firm in perfect competition.

C Marginal cost for a product

$\epsilon \quad$ Very small non-negative number between 0 and 1.

Note 1: $\quad$ All prices and costs are defined per single SU for a finite block of $B$ bytes with the unit ' $\$ / B$ ' for NRT delay insensitive applications, while for RT delay sensitive applications prices and costs are defined in terms of fixed time interval $t$ seconds with the unit ' $\$ / t$ '. It must be noted that the monetary unit considered throughout this thesis is dollars denoted by ' $\$$ '.

Note 2: Chapter 5 considers WSPs with a single BS, and hence the same index $i$ is used for the WSPs for clarity of analysis. 


\section{Chapter 1}

\section{Introduction}

The phenomenal advancements in wireless telecommunication technology in congruence with the growth of the internet has given rise to to advanced applications with higher data rates, thus rendering the radio spectrum as an extremely scarce resource. However, spectrum measurement studies conducted in [1] revealed the underutilization of this radio spectrum in both spatial and temporal domains as shown in Fig. 1.1 taken from [1].

This underutilization presented a serious challenge for the government and national spectrum regulating bodies responsible for regulating the spectrum to satisfy the ever increasing demand for mobile connectivity. With the goal of efficient, intelligent and fair use of this scarce but expensive radio spectrum, the Federal Communications Commission (FCC) issued the Spectrum Policy Task Force (SPTF) report in 2002 [2]. The SPTF report suggested the replacement of the current rigid 'command and control' spectrum policies with new flexible spectrum policies under the Dynamic Spectrum Access (DSA) paradigm. This DSA paradigm provided a tremendous impetus for research in the development of flexible spectrum policies for the efficient utilization of the radio spectrum, by allowing unsubscribed Secondary User (SU) access.

This chapter introduces the DSA techniques and presents the rationale for DSA in infrastructure based networks considered in this thesis. This chapter is organized 


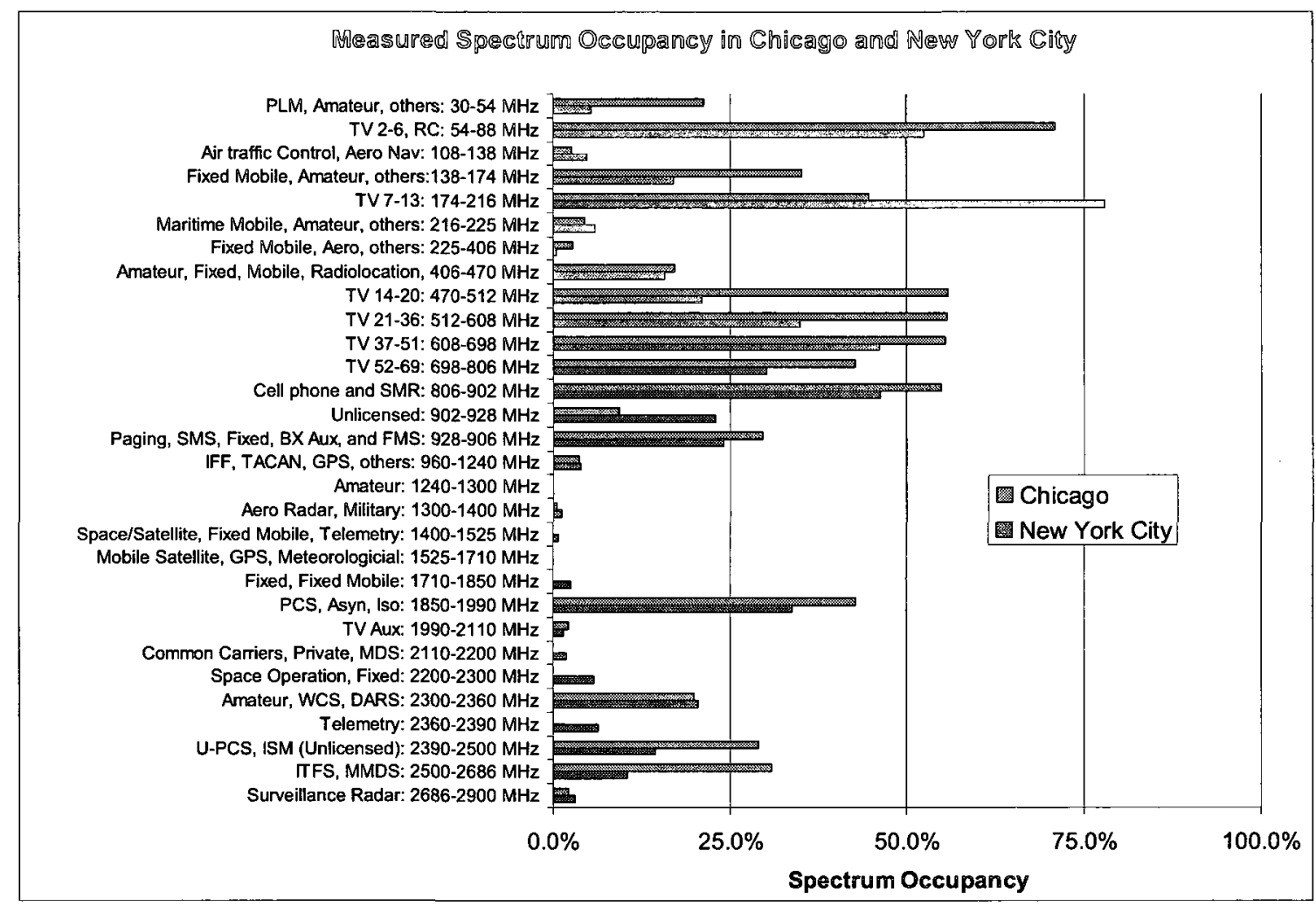

Figure 1.1: Spectrum measurements at peak hours on weekdays

further as follows: Section 1.1 introduces DSA techniques, and Section 1.2 presents the rationale, the thesis goal and the structure of this thesis. The contributions from this thesis are listed in Section 1.3 and Section 1.4 presents a detailed organization of this thesis. The published, submitted and in progress manuscripts based on the work in this thesis are listed in Section 1.5.

\subsection{Dynamic Spectrum Access Techniques}

DSA techniques enable the efficient utilization of the radio spectrum by allowing access for unsubscribed SUs to the white spaces or spectrum holes in the radio spectrum [3-5]. These white spaces or spectrum holes are defined as parts of the radio spectrum unutilized at a particular time and location [4]. The key difficulties in en- 
abling SU access arise from the interference and possible distortion of service to the Primary Users (PUs), who can be considered as the subscribed users or the priority users in that spectrum band. The DSA policies allowing SU access to the temporarily unutilized part of the spectrum thus need to ensure safe, secure and uninterrupted access to the PUs [2-5].

This necessity of safeguarding the PU service requires the SU terminals to have a decision making capability based on DSA policies defined for their operation in the chosen spectrum band. Intelligent SU terminals aptly named as Cognitive Radios (CRs) have been envisioned to be the enabling technology for DSA.

\subsubsection{Cognitive Radio and Current Mobile Device Trends}

CRs are defined to have the capabilities to scan and detect spectrum holes, making them spectrum aware, while simultaneously tracking the channel conditions, making them environment aware $[6,7]$. The CRs are also envisioned to have policy based mechanisms for decision making based on the spectrum availability and channel state information. The ability to reconfigure their hardware and software with respect to Radio Access Technology (RAT) for the opportunistic occupancy of the white spaces in any spectrum band is also an essential requirement for the CR terminals $[4,6,7]$.

To aid the idea of reconfiguration to any spectrum band, the CRs are envisioned to be based on the Software Defined Radio (SDR) platform. These SDRs are conceptualized to be implemented $80 \%$ in software and $20 \%$ in hardware, unlike current mobile devices which generically contain $80 \%$ hardware and $20 \%$ software [6]. With different RATs implemented in software on general purpose processors, the necessary software patches with respect to a particular RAT are envisioned to be downloaded 
whenever required, thus providing multi-RAT (multi-mode) functionality to the SUs for enabling flexible global operation $[3,4,6]$.

The current industry trend however can be seen to approach the envisioned cognitive capability and reconfigurability through a different direction with smartphones supporting multiple RATs on separate chips already flooding the market ${ }^{1}$. These latest smartphones support 2G technologies such as GSM/EDGE networks in the $800,850,1800,1900 \mathrm{MHz}$ frequency bands as well as $3 \mathrm{G}$ technologies such as UMTS/HSPA in the $850,1900,2100 \mathrm{MHz}$ frequency bands. Apart from just $2 \mathrm{G}$ and 3G technology support, these devices also support WiFi (IEEE 802.11) networks [8]. Although these current smartphones are programmed for BS-subscriber master-slave operation, basic decision making capabilities coupled with spectrum scanning and RAT reconfiguration capabilities, can potentially transform these smartphones for CR-like operation in the future.

\subsubsection{Classification of DSA Techniques}

With smart CR like SU terminals for operation, the DSA paradigm can be explored in a variety of ways with the goal of achieving intelligent, efficient and fair utilization in the spectrum band of operation. Thus based on spectrum band of operation, DSA techniques can be classified broadly into two basic types:

- Licensed spectrum bands

- Unlicensed spectrum bands

The other type of classification for DSA techniques can be based on the network structure as shown below:

\footnotetext{
${ }^{1}$ Iphone from Apple, Blackberry Storm from RIM, Omnia from Samsung, N900 from Nokia, etc. [8]
} 
- Infrastructure based networks

- Ad-hoc networks

Within the above classification, further subclassifications are also possible based on power levels or types of architecture. The above classifications are aimed at clearly identifying the scope and the focus of the work presented in this thesis.

\subsection{Rationale and Thesis Goal}

Wireless Service Providers (WSPs) invest heavily in licensing the radio spectrum for providing wireless access to their subscribers in infrastructure based networks. The dynamic nature of radio spectrum usage characteristics in these licensed bands arises from the variable usage by the subscribed $\mathrm{PU}$ s with respect to time and location.

The underutilization of this expensive resource can be effectively translated into wastage of the precious resource. It must be noted that the WSPs pay a hefty price for the licensed spectrum, and thus the underutilization of this resource directly impacts the WSPs' bottom line implicating reduction in profits. Providing temporary access to unsubscribed SUs from the currently underutilized spectrum at the Base Stations (BSs) of the WSPs, can thus potentially generate additional profits for the WSPs. However, enabling such SU access requires technological and economic analysis to establish the mutual benefit of temporary wireless connectivity to the SUs and the potential for additional profits to the WSPs from such SU access.

The goal of this thesis is to develop a distributed system framework, which enables a $\mathrm{SU}$ to pay and obtain temporary wireless access at any time, and any location wherever wireless coverage is available, without the need to subscribe to a WSP. Thus this thesis has two goals: the technological goal is to develop a system framework 


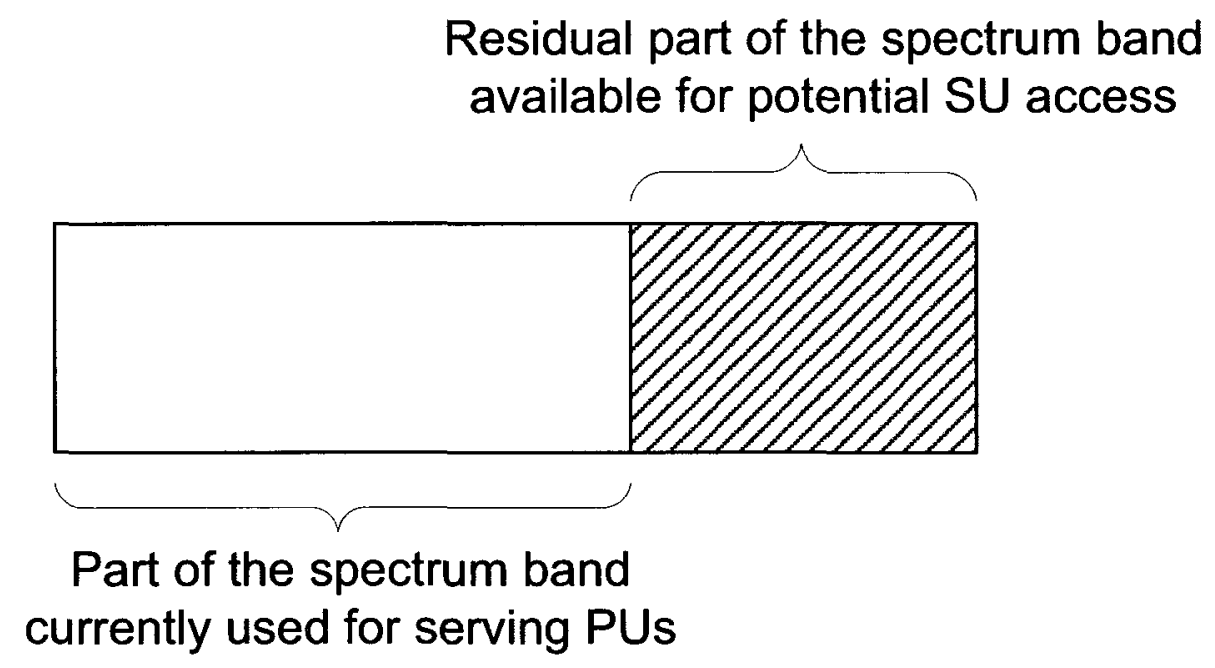

Figure 1.2: Snapshot of the spectrum band licensed by the WSP at the BS

for enabling unsubscribed SU access to the BSs of the WSPs, whenever unutilized spectrum is available at the BSs, and the economic goal is to supplement the system framework by demonstrating the potential for additional profits to the WSPs from such SU access.

\subsubsection{Proposed Distributed System Framework}

Considering a single frequency network, the entire spectrum licensed by the WSPs is available at each BSs. Figure 1.2 shows a snapshot of the spectrum licensed by the WSPs available at a BS, where the plain blue region represents the PUs currently being served at the BS and the shaded orange region represents the residual spectrum currently unutilized at the BS.

The goal of this thesis is to present a system framework, which can make this 
residual spectrum at the BS available to the SUs for temporary wireless access, and provide the WSPs additional profits from this SU access. The proposed framework for PU-SU spectrum sharing presented in this thesis is based on orthogonal coexistence of PUs and SUs at the BSs of the WSP. Previous works such as $[9,10]$ have also suggested PU-SU coexistence, but with reuse of the frequency bands and thus the SUs are required to operate at lower power levels for avoiding interference to PUs. Orthogonal coexistence in this thesis refers to the orthogonal assignment of frequency, time or code (depending on the RAT) to PUs and SUs through efficient Radio Resource Management (RRM) at the BS, thus avoiding interference from SUs to PUs. Although orthogonal joint PU SU coexistence may lead to adjacent channel interference as the load on the system increases, such interference can be reduced by effective radio resource management and coding at the BS and is considered to only have a marginal or negligible impact on the system.

It must be noted that, with regard to the framework presented in this thesis, the SUs are allowed temporary wireless access without any deterioration to the PU service unlike in [11]. Since the PU demand can fluctuate widely due to the use of high data-rate applications, it may not be possible for the WSPs controlling the BSs to guarantee Quality of Service (QoS) to the SUs at all times.

The proposed framework is considered distributed, since the WSPs and their BSs are assumed to have independent decision making capabilities for allowing SU access, with respect to the residual spectrum at a given BS. BSs with increased functionality have become a part of the $4 \mathrm{G}$ network architecture in LTE/LTE-A [12] and WiMAX [13], as a result of the removal of the Radio Network Controller (RNC).

This thesis is aimed at presenting a system framework for enabling SU access at the BSs of WSPs in infrastructure based networks, considering the technological 
feasibility as well as the economic viability of the system. The thesis structure based on the technological and economic aspects is described next.

\subsubsection{Thesis Structure}

From the implementation perspective, the WSPs need to modify the current infrastructure to enable SU access. The WSPs thus face the question: "Will the profits from enabling SU access be greater than the investments costs in infrastructure enhancements, spectrum lease and current infrastructure?"

The economic viability for this framework is thus based on technological feasibility and the costs for infrastructure enhancements. Since current spectrum policies do not allow WSPs to enable SU access, the WSPs need to analyze the potential benefits before enabling SU access. The goal of this thesis is to follow the dotted path in Fig. 1.3 highlighting the technological and economic promise for SU access in future wireless networks, based on the proposed system framework. The topics focused in the respective chapters are indicated within the circles in Fig. 1.3.

\section{Technological Aspect}

A distributed system framework for dynamic spectrum access to secondary users in the licensed bands for infrastructure based wireless networks is proposed in this thesis, with the consideration of minimum modifications to the WSP infrastructure and the SU terminal. Technological aspects such as the signaling framework for unsubscribed SU access, SU identification at the BS, PU-SU RRM at the BS, SU terminal initiated network selection and a price-based handoff scheme are proposed in this thesis.

Efficient utilization of the radio spectrum at the BSs of the WSPs can be achieved 


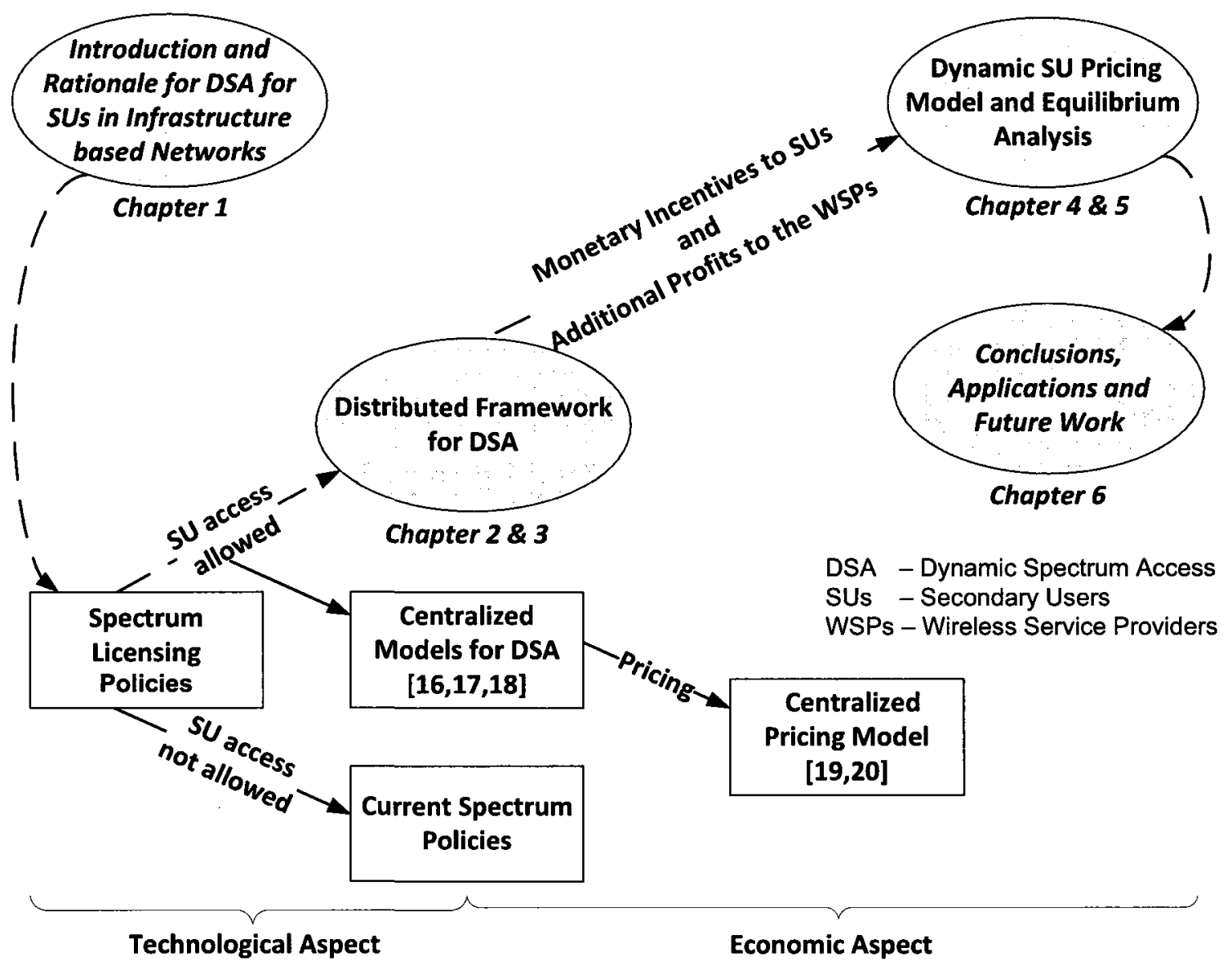

Figure 1.3: Technological and economic aspects - structure of the thesis

by allowing SU access based on system framework proposed in this thesis for infrastructure based networks. Chapters 2 and 3 focus on the technological aspects at the WSP BS and the SU terminal respectively.

Economic Aspect

Based on the proposed distributed system framework, the economic aspect is aimed at presenting a business case for the WSPs to potentially generate additional revenue from SU access. The economic aspect includes a separate dynamic pricing model for charging SUs, which offers incentives to SUs for obtaining temporary wire- 
less access during periods of low PU usage at the BS. The key feature of the pricing model is that it inherently performs SU admission control, thus restricting SU access as $\mathrm{PU}$ demand at a given $\mathrm{BS}$ increases.

This pricing model is then extended for considering inter-WSP pricing competition among multiple WSPs in the region. Non-cooperative game theory based equilibrium analysis with differentiation of SU wireless service is used for achieving inter-WSP competitive pricing, along with the maximization of individual WSP profits from SU service. Chapter 4 and 5 focus on the economic aspects based on the dynamic SU pricing model and equilibrium analysis respectively.

\subsubsection{Scope of the Thesis}

This thesis is aimed at pursuing the problem of efficient spectrum utilization for the licensed bands in infrastructure based networks considering no ad-hoc operation for the SUs. The emergence of DSA techniques for better spectrum utilization sparked a need for the development of market structures for wireless services. References [14-16] presented potential market models aimed towards the successful implementation of the DSA paradigm in the wireless technologies of future. The motivation for this thesis is thus based on exploring the technological and economic feasibility for providing additional profits to the WSPs from SU access considering minimum modifications to the current WSP infrastructure.

This thesis is primarily focused at developing a distributed framework for providing unsubscribed SU access by selling the unutilized spectrum at the BSs of the WSPs. Thus it must be noted that, this thesis is not about performance analysis, but presents a system framework with the joint implementation of a variety of system elements in conjunction with wireless service market dynamics for providing access 


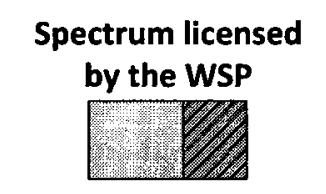

Enhanced BS:

All-IP based BSs with autonomous decision making capability
At the Base Station
+ Model for joint PU-SU resource management

+ SU identification based on the using the DSCP byte

+ Economic Aspect: Incentive based pricing model for SUs based on PU demand and reference $P U$ price
+ Proposed Distributed Framework

+ Orthogonal coexistence of PUs and SUs at the same BS.

+ No deterioration to $P U$ service.

+ Temporary wireless access to SUs.

+ Additional profits to WSPs from SUs.

+ Standalone operation of the WSPs, without the need for spectrum pooling and cooperation with other WSPs

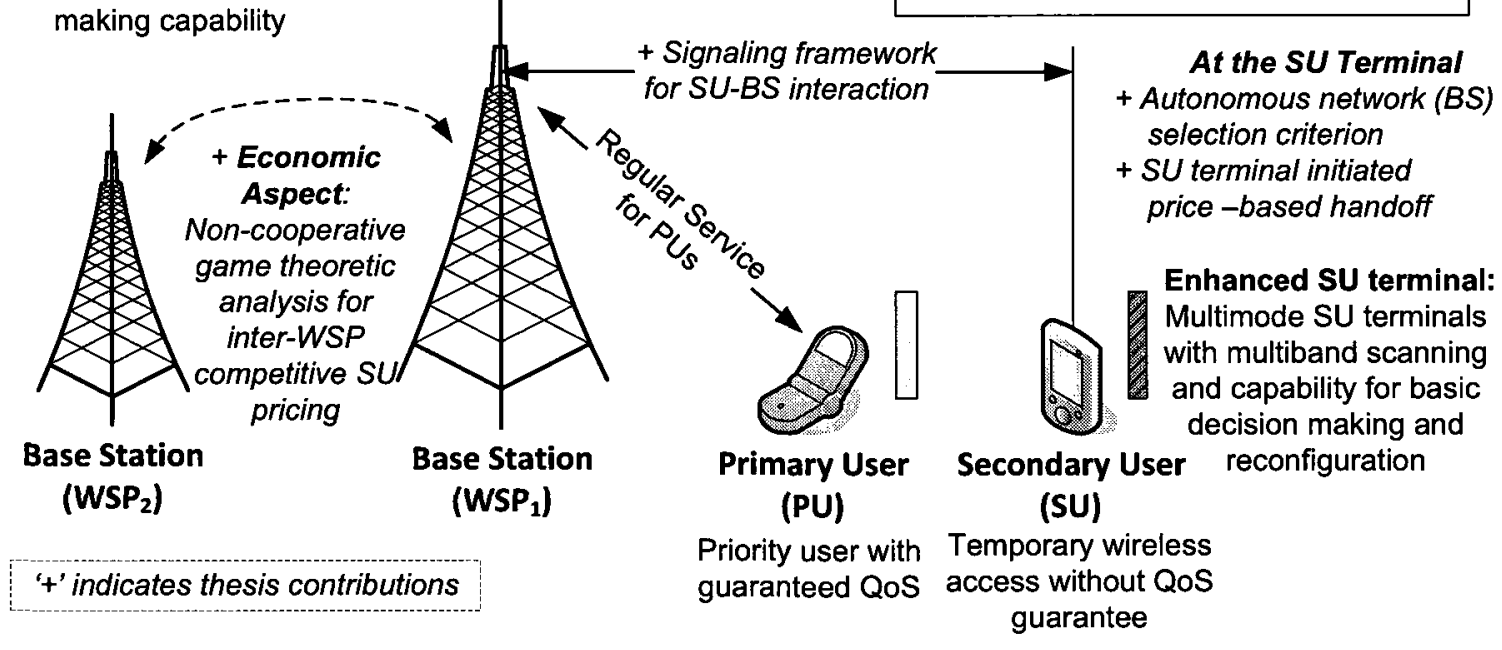

Figure 1.4: Thesis contributions

to unsubscribed SUs.

\subsection{Thesis Contributions}

This thesis is intended to provide a systems perspective from the technological and economic perspectives. The distributed framework proposed in this thesis enables the orthogonal coexistence of PUs and SUs at the BS in contrast to $[9,10]$. The distributed framework thus allows individual WSPs to provide access to the SUs directly, without the need for centralized entities and cooperation with other WSPs, and is a major shift from previous works in literature, e.g., [16-20]. In an effort to realize the proposed framework, the various contributions from this thesis are listed 
below and illustrated in Fig. 1.4:

Proposed Modifications at the Base Station The BS is assumed to have enhanced decision making capabilities similar to those proposed in LTE/WiMAX $[12,13]$ in $4 \mathrm{G}$ networks. In order to allow $\mathrm{SU}$ access to the unutilized spectrum at the BS of a WSP, the BSs need to be enriched with the following features.

- SU identification based on the Differentiated Service Code Point (DSCP) byte from the IP header.

- Dynamic incentive based pricing model for charging SUs.

- RRM model for coexistence of PUs (subscribed) and SUs (unsubscribed) at the BS.

Proposed Operational Functionality at the SU Terminal The SU terminals are assumed to have multimode operation with frequency band scanning capability for detecting spectrum information, as well as the ability to reconfigure to any RAT provided by the BSs of the WSPs. To select a BS and consequently the WSP most suitable for the SU, the following decision making capabilities are enabled at the SU terminal.

- Criterion for autonomous BS selection at the SU terminal, based on price charged to SUs by the WSPs and signal strength at the SU terminal.

- SU terminal initiated price based handoff, assuming regular handoff is not required.

The SU-BS Link The SU-BS link is the essential part of the proposed framework without which unsubscribed SUs cannot access the BS of a WSP. A signaling framework based on the following features is developed in this thesis. 
- SU-BS interaction for linking the unsubscribed SUs with the BS of the WSP.

- Authentication of the SUs requesting temporary wireless access to the selected BS of a WSP.

- Data and control signaling between SUs and the selected BS after connection establishment.

Non-cooperative Inter-WSP Competitive SU Pricing Establishing the economic viability and thus a business case for the proposed framework based on the features listed below is the key economic contribution in this thesis.

- Dynamic incentive based pricing for SUs based on PU demand.

- Equilibrium analysis with differentiated SU wireless service based on noncooperative game theory for achieving inter-WSP competitive pricing.

- Dual goal of discounted SU pricing along with additional profits to WSPs.

Although the contributions are categorized with respect to the architectural blocks of the system, the organization of the chapters in this thesis is slightly different and is elaborated in the next section.

\subsection{Organization of the Thesis}

Since various technological and economic aspects of the system are considered in this thesis, a brief survey of the literature is presented at the beginning of every chapter for better clarity. This thesis is organized as follows:

- The proposed system framework for unsubscribed SU access is presented in Chapter 2. The various technical aspects related to SU-BS interaction as well 
as management of PUs and SUs at the same BS are described in this chapter. This chapter focuses on the operations at the BS of the WSP for enabling SU access.

- Chapter 3 deals with the functioning at the SU terminal, where the SU terminal selects a particular BS of a WSP for temporary wireless access. The network (BS) selection criterion at the SU terminal is aimed for autonomous operation, without the need for manual intervention. A scheme for price based handoff is also described in this chapter.

- Chapter 4 focuses primarily on the pricing mechanism for SUs. The incentive based pricing model for charging SUs, with an inherent property for SU admission control at the BS, is presented in this chapter. The incentive based pricing model provides discounted prices for SU during off-peaks hours, with the aim of revenue generation by attracting SUs to occupy the underutilized network capacity at the BS.

- Chapter 5 demonstrates the economic potential for additional profits to the WSPs through SU access. Equilibrium analysis with SU wireless service differentiation is aimed at achieving competitive pricing among WSPs.

- The conclusions and applications from this thesis, along with the future work extending the ideas from this thesis, are highlighted in Chapter 6.

\subsection{Manuscripts Published, Submitted and In-progress}

In this section, a list of the manuscripts published, submitted or in-progress based on the work in this thesis is presented. 
- Soumitra Dixit, Shalini Periyalwar, and Halim Yanikomeroglu, "A distributed framework with a novel pricing model for enabling dynamic spectrum access for secondary users", in Proceedings of the IEEE Vehicular Technology Conference (VTC2009-Fall), 20 - 23 September 2009, Anchorage, Alaska, USA. The work in this paper is based on Chapters 2, 3 and 4 from this thesis.

- Soumitra Dixit, Shalini Periyalwar, and Halim Yanikomeroglu, "Equilibrium analysis with differentiated pricing for secondary users in wireless networks". This is the working title for a paper in progress based on Chapters 2, 4, and 5 . This paper is aimed for a conference submission.

- Soumitra Dixit, Shalini Periyalwar, and Halim Yanikomeroglu, "A distributed framework for enabling dynamic spectrum access for secondary users with a novel pricing model and inter-WSP competitive pricing". The work on this paper is currently in progress and is based on the entire thesis. This paper is aimed for a journal submission. 


\section{Chapter 2}

\section{System Framework}

The distributed system framework for $\mathrm{SU}$ access is introduced in this chapter, with the focus on the technological aspect. The enhancements at the BS of the WSP for enabling SU access have been proposed considering minimum modifications to the existing WSP infrastructure.

In this chapter, a literature review highlighting previous architectures for DSA in the licensed bands for infrastructure based networks is presented in Section 2.1 . Section 2.2 describes the motivation for the proposed framework along with the key features, and Section 2.3 gives the system model. The signaling framework for BSs to interact with the unsubscribed SUs is described in Section 2.4 and a mechanism for SU identification based on the DSCP byte is proposed in Section 2.5. Section 2.6 presents a structure for joint PU-SU RRM at the BS. The summary of this chapter is given in Section 2.7 .

\section{$2.1 \quad$ Literature Review}

\subsubsection{Classification of Licensed Band Spectrum Sharing Techniques}

DSA or spectrum sharing techniques for the licensed bands in infrastructure based networks can be broadly categorized as follows:

Category 1: Spectrum sharing among WSPs This type of spectrum sharing 
allows the WSPs to borrow spectrum from each other temporarily, thus increasing the network capacity.

Category 2: Spectrum sharing among PUs and SUs This spectrum sharing category includes techniques for management of SUs along with PUs in the licensed spectrum bands. Spectrum in this category is directly sold to the SUs requesting access.

A clear division between these two categories has been presented here, with the aim of positioning work presented in this thesis with respect to the previous works from literature.

\subsubsection{Centralized Architectures}

The most common approach for DSA in infrastructure based networks has been based on the idea of spectrum pooling, which was explored in [16]. The key idea presented in [16] was that a centralized spectrum pool could be created by collecting together the unutilized spectrum in different spectrum bands. The local WSPs could then rent a part of this spectrum temporarily for a fee, thus temporarily increasing their capacity. The key challenges related to this approach are the regulatory policies and architectures to enable DSA with spectrum pooling. The spectrum pooling and spectrum information pooling approaches have been studied in both the spectrum sharing categories and primarily fall under centralized architectures.

The key element generally common to any spectrum pooling based centralized architecture is presence of a Centralized Mediating Entity (CME) to enable spectrum pooling and regulation, co-ordination among WSPs $[17,18]$, or negotiate spectrum access opportunities directly with the SUs $[19,20]$. These centralized architectures 


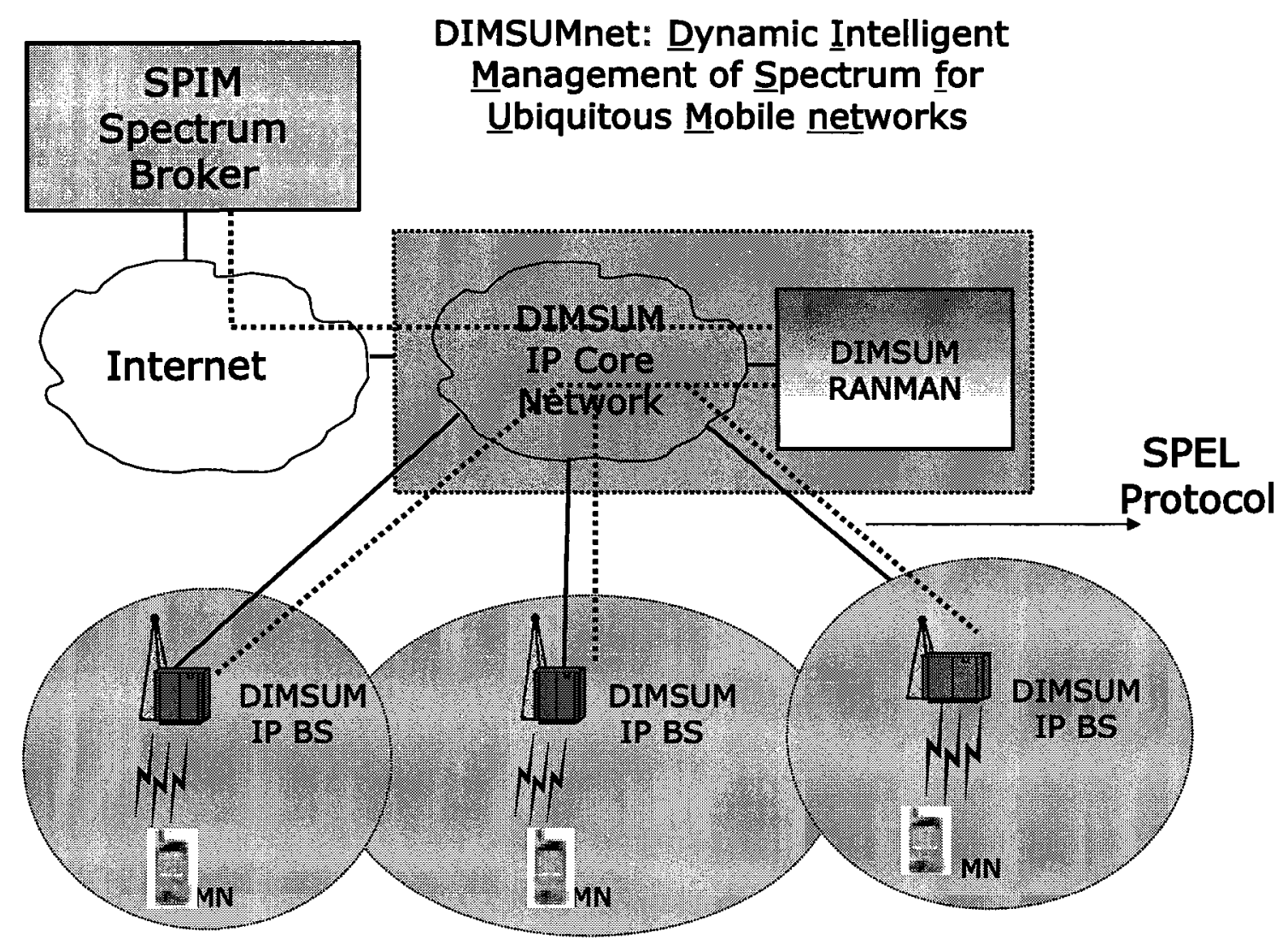

Figure 2.1: DIMSUMnet architecture

often require multiple regional CMEs since the radio spectrum usage characteristics vary with time and location. A few examples are presented next to demonstrate the various features and functionality of centralized DSA architectures.

\section{DIMSUMnet Architecture [17]}

Reference [17] proposed the Coordinated Access Band (CAB) approach, where CAB is a spectrum pool to be reserved by the regulation bodies for controlled DSA. The DIMSUMnet architecture, an acronym for Dynamic Intelligent Management of Spectrum for Ubiquitous Mobile Networks [17] exploits the CME called the spectrum broker for spectrum regulation, coordination and sharing of the CAB among WSPs 
in the area for temporarily increasing their capacity. A direct spectrum leasing approach, where the SUs directly interact with the spectrum broker is also considered in the advanced model.

New infrastructure elements such as spectrum broker and the radio access network manager, along with new protocols for their functioning are proposed in [17] as shown in Fig. 2.1 taken from [17]. However, with the WSPs already having invested heavily in leasing the radio spectrum and existing infrastructure, adding new architectural blocks and protocols to enable DSA may become a heavy burden for the WSPs to support, since the proposal in [17] involves major modifications to the current infrastructure.

Spectrum Information Pooling with On-demand Cognitive Pilot Channel [18]

The Cognitive Pilot Channel (CPC) was proposed as a part of the End-to-End Reconfigurability (E2R) project [21] in the $6^{\text {th }}$ Framework Programme (FP6) for research on future wireless networks under the European Union. The development of the CPC was aimed for enabling unsubscribed SUs to select an appropriate WSP to fit their requirements based on spectrum information conveyed to them over a common channel. In order to provide the SUs with collated spectrum information from all the WSPs, the CPC was conceptualized to operate on a universally harmonized frequency for allowing the flexibility for CR equipped SUs to operate globally [21].

The concept of CPC was adopted in [18], where a novel scheme for transfer of spectrum information on-demand to the SUs based on the on-demand CPC was proposed as shown in Fig. 2.2 taken from [18]. The CPC access scheme shown in Fig. 2.2 is a hybrid of in-band and out-band CPCs. The CR equipped SUs initially gain basic WSP related information on the universally harmonized out-band CPC only 


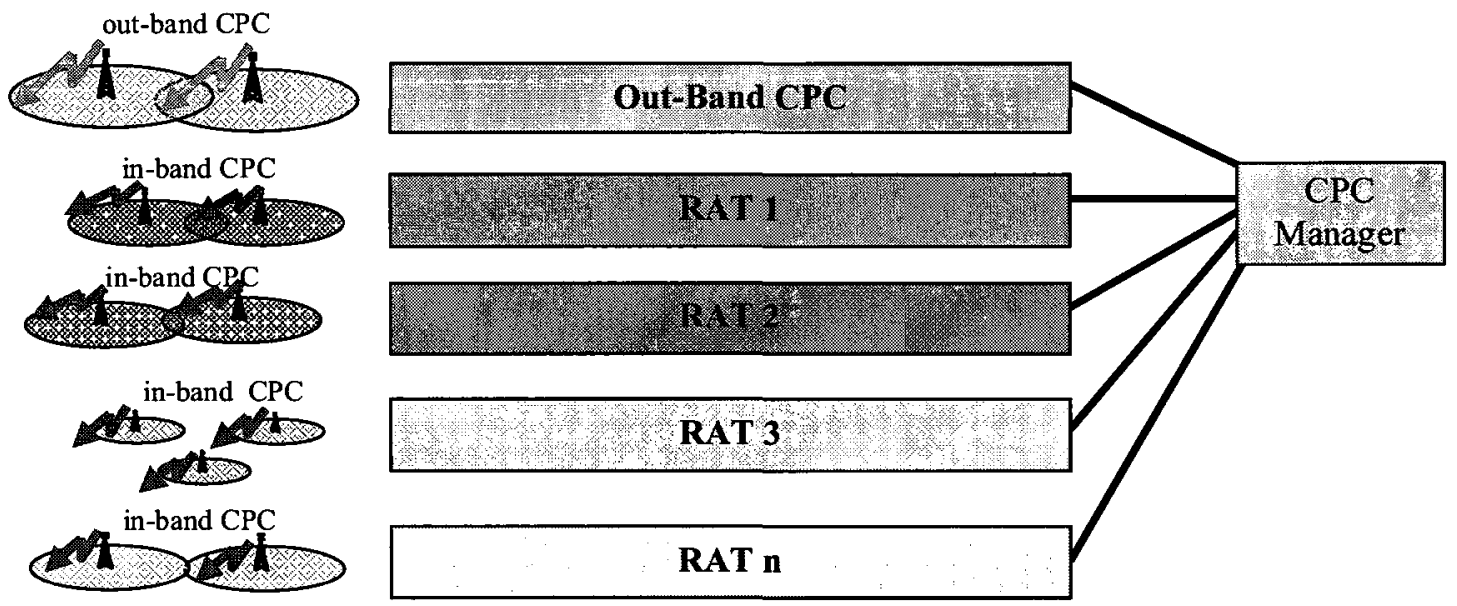

Figure 2.2: Hybrid CPC access scheme

when requested by the SUs. Based on the basic WSP related information obtained by the SUs through the on-demand out-band CPC, the SUs can access the in-band CPC where detailed information related to every WSPs in the region transmitted.

The CPC manager provides collated spectrum information collected from the local WSPs over the CPC. Although the schemes proposed in [18] are useful in passing collated spectrum information to the SUs thus avoiding spectrum scanning at the SU terminal, multiple regional CPC managers are required to transfer the local spectrum information collected from the regional WSPs, due to dynamics of radio spectrum usage. The cooperation and coordination among the WSPs in the region is also essential for the successful implementation of this scheme.

\subsubsection{Distributed Architectures}

The centralized architectures have been very dominant for DSA in infrastructure based networks, while distributed architectures architectures are generally considering for ad-hoc networks which involve cooperative sensing of the radio spectrum $[4,22]$. SUs using DSA techniques involving cooperative sensing [22] need to search for white 
spaces in the radio spectrum for opportunistic access $[3,4,22]$.

However, in infrastructure based networks, $[9,10]$ have proposed frameworks, which could be considered as distributed. These frameworks allow the coexistence of PUs and SUs, where the SUs are made to operate at lower power levels due to the reuse of frequencies with the PUs.

\subsection{Motivation for the Proposed Framework}

One of the drawbacks in $[17,18]$ arises due to the variable usage of the radio spectrum with respect to time and location. The requirement of regional CMEs, real time operation with fast coordination among WSPs or between CME and SU require specialized protocols for their implementation, and hence these centralized approaches may present a major overhead in signaling and coordination, to the WSPs.

It is also unreasonable to assume cooperation of all the WSPs to share their spectrum information, owing to the fierce competition among WSPs. This requirement of cooperation among WSPs for running CME based spectrum pooling operation also restricts any independent WSP from providing SU access. The proposed framework is thus called distributed, since the BSs make localized decision related to SU access without the use of CMEs.

Although, the proposed framework is called distributed, it is different from cooperative sensing approach in [22] since infrastructure based networks are considered in this thesis. Also the orthogonal coexistence of the SUs along with PUs is assumed at the BS, unlike $[9,10]$.

The proposed distributed framework thus strictly falls under the category type two spectrum sharing, where radio spectrum is directly sold to the SUs by the WSPs and does not include CMEs. Thus this distributed framework is a novel framework, 
which provides SU access and additional WSP profits with minimum modifications to current infrastructure, where PU-SU co-exist orthogonally. The key features and the system model for the proposed framework are presented in the next section.

\subsubsection{Key Features}

The key features of the proposed distributed system framework for DSA to SUs in infrastructure based wireless networks are as follows:

- The proposed system framework offers temporary wireless access to SUs and provides additional profit to WSPs from SU access to the unutilized spectrum at the BSs of the WSPs.

- The proposed distributed framework is based on the idea that allows the orthogonal coexistence of PUs along with the SUs for functioning at the same BS of the WSP, assuming efficient RRM at the BS.

- The system framework provides profitable and efficient utilization of the spectrum licensed by the WSPs in the spatial and temporal domains, through SU access.

- The system framework is proposed considering minimum modifications to current WSP infrastructure and to SU terminals in providing unsubscribed SU access.

- Spectrum sharing among different WSPs through the spectrum pooling approach using a CME for temporarily increasing their capacity, is not considered as a part of the framework. The distributed framework does not require 
CMEs for coordination and cooperation among WSPs as in [17-20] for sharing spectrum and spectrum information.

- The proposed distributed framework thus allows standalone WSPs to offer direct access to the SUs, without CMEs and without the need for cooperation with other regional WSPs.

On examining the key features of the system framework, it can be seen that the proposed distributed framework is significantly different from the centralized architecture with CME [17-20] and the distributed architecture as described in $[4,9$, 10,22]. The distributed framework proposed in this thesis can be considered as an intermediate step between the current infrastructure based wireless networks and the cognitive radio networks envisioned for the future.

\subsection{System Model}

The wireless telecommunications industry is evolving towards $4 \mathrm{G}$, with new RATs such as Long Term Evolution (LTE, LTE-A) [12] and WiMAX [13] based on Orthogonal Frequency Division Multiplexing (OFDM), packet switching and an all-Internet Protocol (IP) based framework. The removal of RNCs from 4G architecture [12,13] has been the enabler for enhanced capabilities at the BS enabling autonomous decision making and resource allocation. This enhanced decision making capability at the $4 \mathrm{G}$ BSs has also been the enabler for the proposed distributed framework.

A single frequency network has been considered in the system model such that the entire spectrum licensed by the WSPs is available for usage at every BS. A generic scenario with a single SU in the coverage area of the BSs of three WSPs supporting different RATs is shown in Fig. 2.3. The BSs of the WSPs are connected 


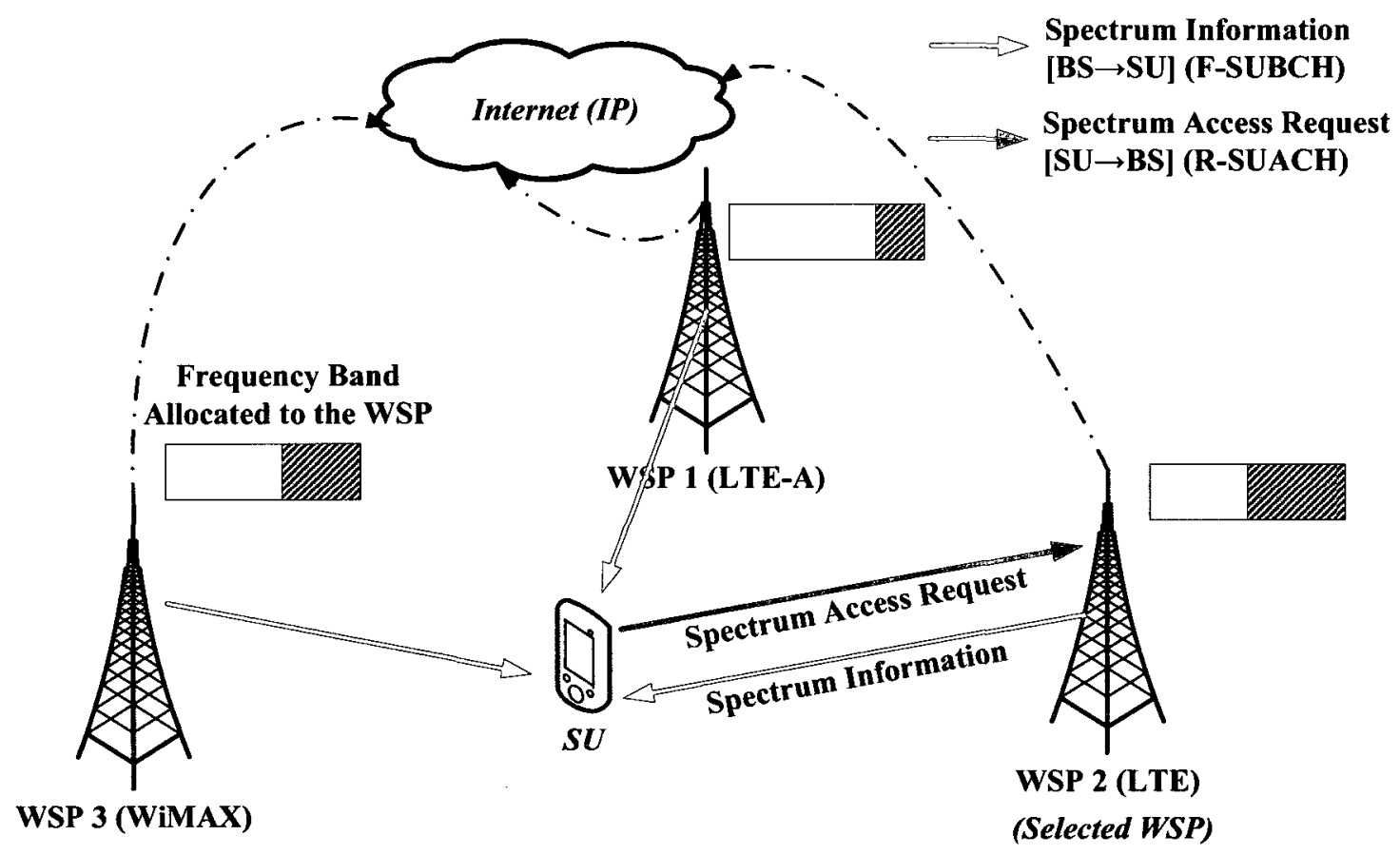

Figure 2.3: Network scenario with SU requiring wireless access

to the internet via dotted lines to indicate the use of an all-IP based framework. The Authentication Server (AS) is also present at the WSPs for authenticating the SUs requesting temporary access, but is not shown in Fig. 2.3.

The framework presented can support either Frequency Division Duplexing (FDD) or Time Division Duplexing (TDD) modes of operation depending on the WSPs' RAT. A single frequency network has been considered in Fig. 2.3, since it is been used in the current 3G-CDMA based technology as well as has been standardized as a part of the $4 \mathrm{G}$ framework $[12,13]$.

\subsubsection{Enhancements for Enabling SU Access}

In oder to enable SU access considering the current infrastructure at the WSPs, the initial SU-BS interaction is based on the channels described next. 
Forward link - Secondary User Broadcast Channel (F-SUBCH)

The distributed framework assumes each BS of a WSP to transmit their local spectrum information over a broadcast channel to the SUs in the area. The spectrum information transmitted by the BS over the Forward link - Secondary User Broadcast Channel (F-SUBCH) to the SUs in the coverage area is detailed in the next section. The SUs can receive spectrum information from all the BSs of the WSPs in the coverage area by scanning the various RAT frequencies to detect the different $\mathrm{F}$ SUBCHs transmitted by each of the BSs providing SU access.

Reverse link - Secondary User Access Channel (R-SUACH)

Detecting the F-SUBCH from the BSs of the WSPs in the coverage area allows the SUs to compare the obtained spectrum information in regard to the SUs' application class requirements, monetary price charged by the WSPs for temporary access and the signal strength at the SU terminal. Based on the spectrum information and the requirements of the SU, the SU send a 'Spectrum Access Request (SAReq)' to the most suitable BS of a WSP over the Reverse link - Secondary User Access Channel (R-SUACH) as shown in Fig. 2.3. The criterion for network (BS) selection at the SU terminal is elaborated in the next chapter. The basic capabilities assumed for the $\mathrm{SU}$ terminal for obtaining unsubscribed $\mathrm{SU}$ access are described in the next subsection.

\subsubsection{SU Terminal}

The SUs are assumed to be equipped with a multimode terminal with the capability of multiple frequency band operation and reconfigurability. This SU terminal can thus either be considered as multimode multichip terminal with each chip supporting a specific RAT similar to current smartphones, or a multimode SDR with 
RAT implemented in software on a general purpose processor. Both types of SU terminals are required to be enhanced for spectrum scanning, advanced decision making and reconfiguration capabilities.

Scanning and detecting the F-SUBCH from the BSs of the WSPs in the coverage area is essential for $\mathrm{SU}$ terminal to gather spectrum information for network selection. The SU terminal can then reconfigure to the RAT of the selected BS of the WSP, thus enabling SUs with the flexibility of operation globally.

Types of SUs

SUs requiring temporary wireless access can be classified into two types:

1. SU Type A: These SUs are not subscribed to any WSP in the region.

2. SU Type B: These are PUs who convert to SUs to gain wireless access to an application class that they are not subscribed to as PUs. For example, a PU subscribed to voice service with a WSP, could gain temporary access as a SU to data or internet service from any WSP in the region.

Preregistration for the SUs

The SUs of both types A and B are required to be preregistered to an online SU database, before they can be granted temporary wireless access by the WSPs. This SU database should thus be secure and accessible only to the AS of the WSP. The SU database needs to include user information similar to any online user database coupled with critical authentication information including device identity.

The signaling framework required for the BS of a WSP to interact with a SU is described next section. 


\subsection{Signaling Framework for SU - BS Interaction}

The process of connecting a SU to a BS for temporary wireless access is divided into three operational phases as listed below:

\section{Initialization}

2. Authentication

\section{Connection Establishment}

The signaling framework for SU-BS interaction based on a distributed mechanism with FDD is illustrated in Fig. 2.4, Fig. 2.5 and Fig. 2.6. The channels between BS and SU are shown by solid lines, whereas the channels between BS and AS, and AS and Internet (IP), are shown using dashed lines.

\subsubsection{Initialization}

The initialization process requires two essential channels for BS-SU interaction, the F-SUBCH and the R-SUACH as described in Section 2.4.1. Additional channels required for authentication and connection establishment are given in the following subsections.

Functionality of the F-SUBCH

The BSs of all WSPs advertise their unutilized spectrum to the SUs in their area over the F-SUBCH as shown in Fig. 2.3 and Fig. 2.4. Due to the distributed nature of this framework, each BS of every WSP transmits its F-SUBCH on separate frequencies to the SUs in the coverage area. Spectrum information passed over the F-SUBCH should include the following to assist BS selection at SU terminal: 
- 128 bit IPv6 or 32 bit IPv4 address and 48 bit MAC (Medium Access Control) address of the transmitting BS with an open destination address

- BS, WSP identifier and the associated RAT

- Uplink frequency of R-SUACH set by the broadcasting BS for SU response, if chosen by the SU

- Application classes offered by the BS $\left(A_{o, i}\right)$

- Fixed price per static time window for all the application classes offered

- Transmission frequency of the F-SUBCH for further SU reference

Functionality of the R-SUACH

The SUs can select the most appropriate network using a network selection criterion described in Chapter 3 and send a 'Spectrum Access Request (SAReq)' to the selected BS of the WSP over the R-SUACH as shown in Fig. 2.3 and Fig. 2.4. Information sent by the SU to the selected BS should include the following:
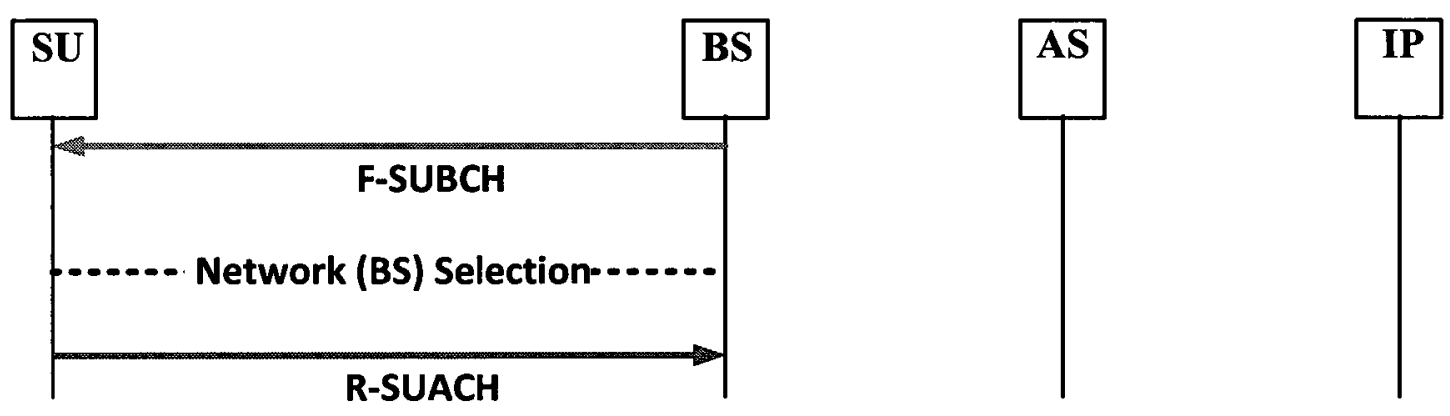

Figure 2.4: Signaling framework: initialization 
- 128 bit IPv6 or 32 bit IPv4 address and 48 bit MAC address of the SU terminal with the packet destination address of the selected BS

- SAReq along with SU authentication information for pricing and billing

- Application class/classes requested by the $\mathrm{SU}\left(A_{r}\right)$

- Channel State Information (CSI) to aid RRM at the selected BS of the WSP

\subsubsection{Authentication}

Upon receiving the SAReq from the $\mathrm{SU}$, the $\mathrm{BS}$ sends a request for authentication to AS of the WSP. On verification of the authentication information from the online SU database, a positive or negative acknowledgment, based on authentication, is then sent to the SU over the Forward link Secondary User Acknowledgment Channel (F-SUAkCH). A temporary service contract will also be sent to the successfully authenticated SU over this channel as shown in Fig. 2.5.

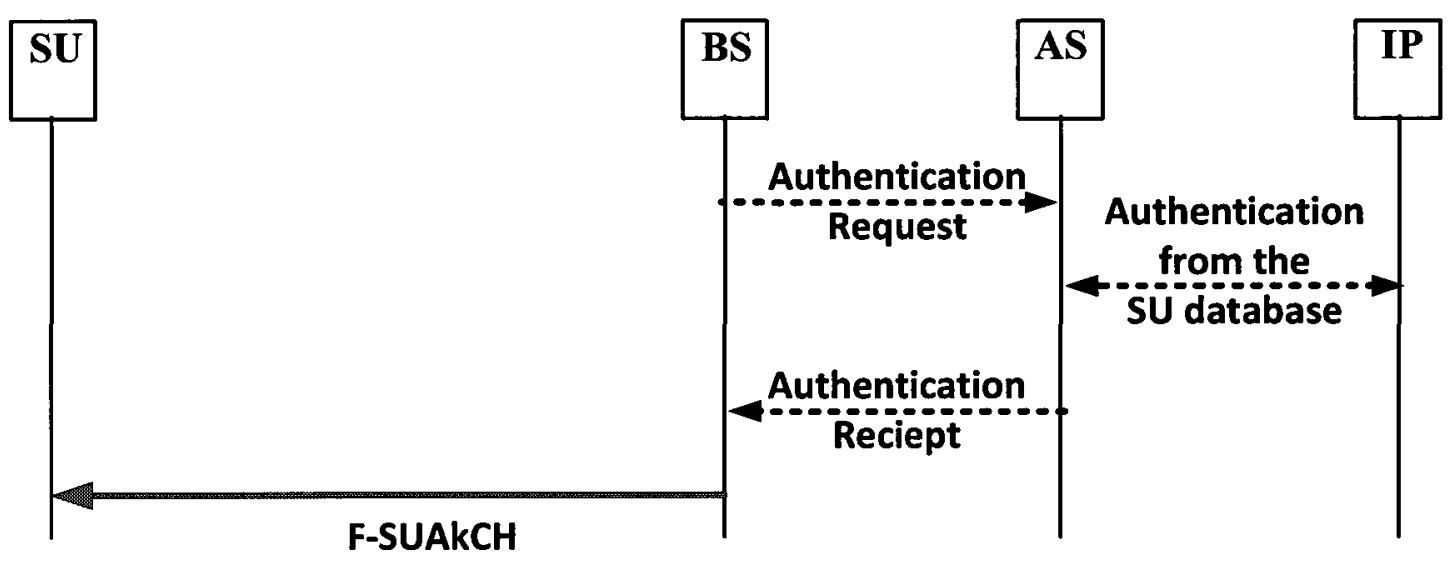

Figure 2.5: Signaling framework: authentication 


\section{SU Service Contract}

Considering the SUs are authenticated by the WSPs and are now eligible for wireless access, details related to the service need to be accepted by the SU before any form of wireless access can be provided. Within the confines of the proposed framework, the following elements must be conveyed as a part of the SU service contract:

- Provision of wireless access on a temporary basis

- No provision for guaranteed QoS (explained in the PU-SU RRM model in Section 2.6)

\subsubsection{Connection Establishment}

After authentication by the WSP, the SU will receive temporary wireless access from the BS of the WSP and follow the signaling protocols as defined by the RAT of the selected BS. The data and control information will then be passed on over the designated channels similar to LTE [12] or WiMAX [13] and is shown in Fig. 2.6. For representation purposes, the downlink data and control channels for passing information from the BS to the SU are named as Forward link-Secondary User Shared Channel (F-SUSCH) and Forward link-Secondary User Control Channel (F-SUCCH) respectively. In the uplink the data and control information is assumed to be passed on from the SU to the BS over the Reverse link- Secondary User Shared Channel (RSUSCH) and Reverse link-Secondary User Control Channel (R-SUCCH) respectively. 


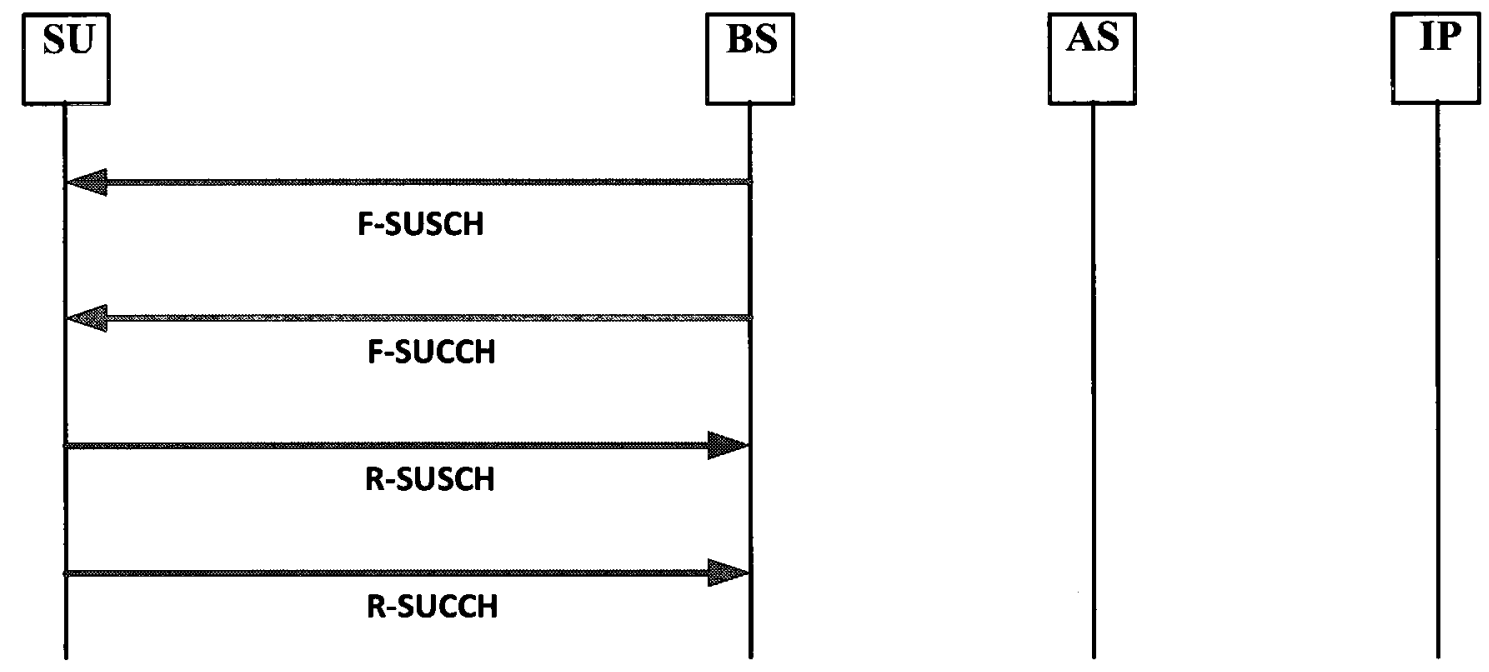

Figure 2.6: Signaling framework: connection establishment

\subsubsection{Modified Signaling Framework for SU Type B}

By definition, the SUs of Type B actually PUs registered to a WSP in the region and convert temporarily to become SUs for connecting to a different application class. Since the PUs are already subscribed to a particular application class, they can receive spectrum information in the form of real time updates over a mechanism analogous to the commercially famous twitter [23]. The twitter service allows real time updates from its users where the information sent and received is limited to 150 characters [23]. Thus spectrum information updates can be received by the PUs over the internet or Short Message Service (SMS) depending on the application class they are subscribed to.

Based on the spectrum information received, the PUs can select the appropriate BS of a WSP for obtaining temporary wireless access to the selected application class as a $\mathrm{SU}$ of type $\mathrm{B}$. The authentication and connection establishment for obtaining SU wireless access to the selected application class will be similar to that explained in Section 2.5.2 and Section 2.5.3 respectively. 

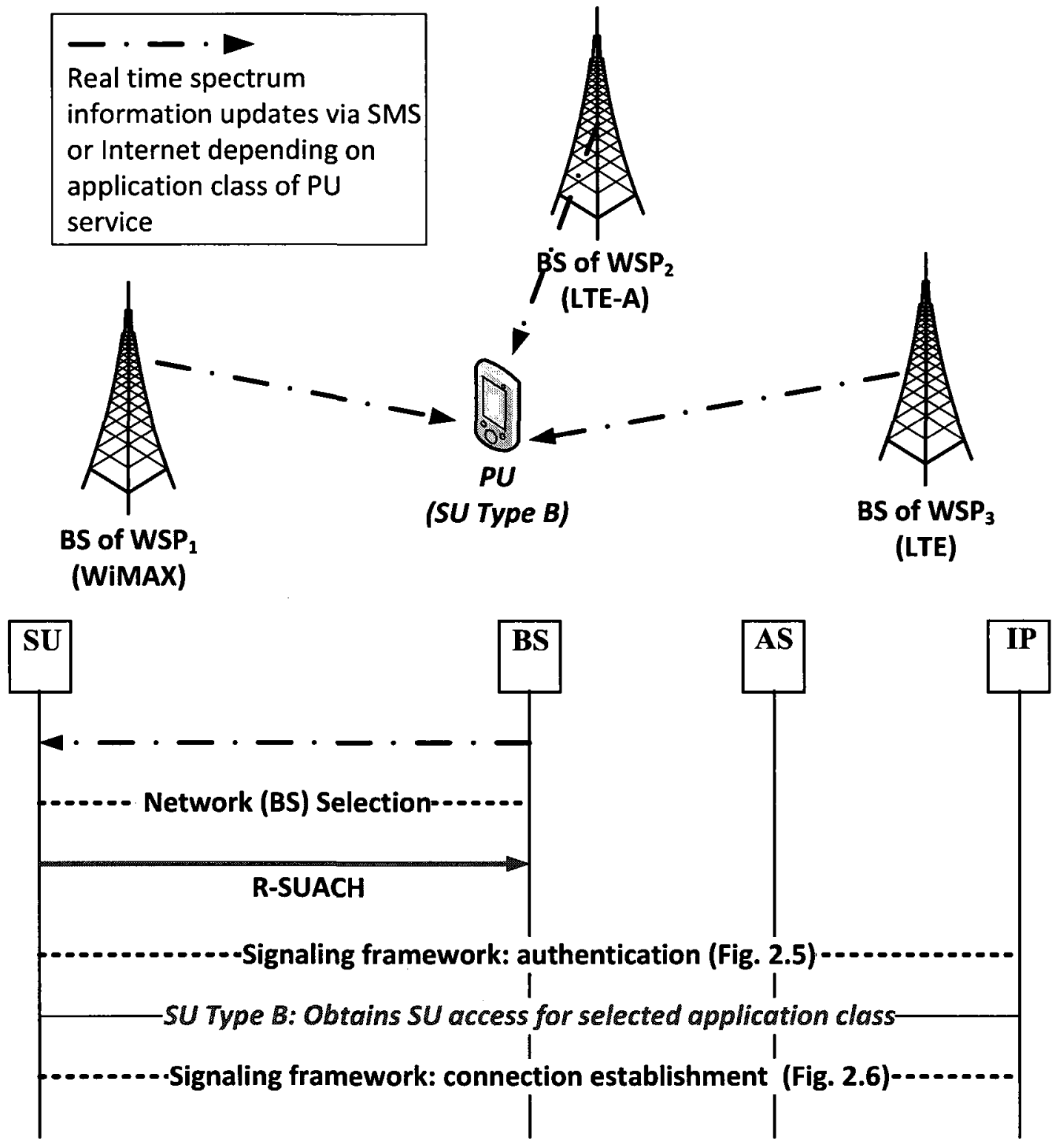

Figure 2.7: Signaling framework: SU type B

This leads to an alternative type of SU-BS initialization as shown in Fig. 2.7. The multi-band scanning at the SU terminal during the initialization phase for each BS of each WSP can thus be avoided, as spectrum information is received via internet or SMS. The PUs would only need to scan the F-SUBCH of the selected BS to verify 
signal detection before sending the SAReq to the selected BS. The modified signaling framework for type B SUs is illustrated in Fig. 2.7

\subsection{Identification of SUs using the DSCP Byte}

The distributed framework proposed in this thesis provides the orthogonal coexistence of PUs and SUs at the same BS in the same spectrum band licensed by the WSP. The PUs and SUs thus need to identified and differentiated at the BS for efficient RRM, SU authentication, and implementation of separate pricing mechanisms. The pricing aspect where the PUs and SUs will have separate pricing models is presented in Chapter 4 .

With the consideration of an all-IP based framework, the PUs and SUs can be identified based on the IP or MAC address; however such implementation may hinder fast and efficient operation at the BS. Even though the SUs may not be guaranteed QoS for Real Time (RT) applications, they need to differentiated from the PUs at the BS based on methods not relying on the QoS specifying bits in the DSCP byte. The use of one unused bit from the DSCP byte of the IP header is proposed for the identification of the SU, which would aid in quick PU-SU differentiation at the BS.

The DSCP byte in the IP header is responsible for indicating the QoS requested by a user. Figure 2.8 shows the DSCP byte defined by Internet Engineering Task Force (IETF) for the Diffserv architecture. The first 6 bits of this byte are used to define the QoS for a particular user, while the last two bits are reserved for future use. These last two bits of the DSCP byte are currently unused and are default set to 0 [24]. The use of the rightmost bit in the DSCP byte is proposed in this thesis for fast differentiation between $\mathrm{PU}$ and $\mathrm{SU}$ at the BS. This rightmost bit can be set to ' 1 ' in the IP packets for the SUs to help differentiate them from the PUs as shown 


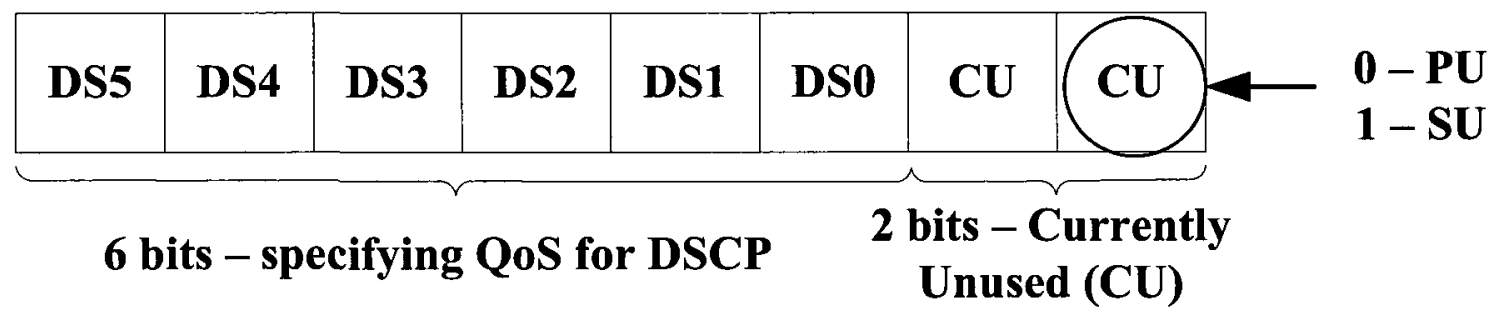

Figure 2.8: DSCP byte and the proposed use of the rightmost bit for SUs

in Fig. 2.8. The decimal value of the DSCP byte thus is odd in case of SU and even for the PU.

Thus the QoS for a particular user, either PU or SU, can be clearly specified through the first 6 bits of the DSCP byte, while the last (rightmost) bit can provide quick differentiation between PUs and SUs at the BS.

The next section details the structure for joint PU-SU RRM at the BS, assuming the PUs and SUs are differentiated based on the use of the DSCP byte.

\subsection{Structure for joint PU-SU RRM at the BS}

As PUs and SUs operate in the same spectrum band at the BS on non-overlapping resources, the RRM model becomes the critical feature of the proposed system framework due to the limited resources available at the BS. The primary goal of joint PU-SU RRM is to allow access to SUs without deterioration of service to the PUs, and a model for such joint RRM is illustrated in Fig. 2.9. To ensure that the PU service is not affected by SU access, the SUs can be provided resources if and only if there exist leftover resources after the PUs have been served. The effect of adjacent channel interference to the PUs from the SUs is assumed to be marginal, and will be tackled by coding and efficient resource and interference management at the BS.

The RRM policy scheduler illustrated in Fig. 2.9 is used for explaining the joint 


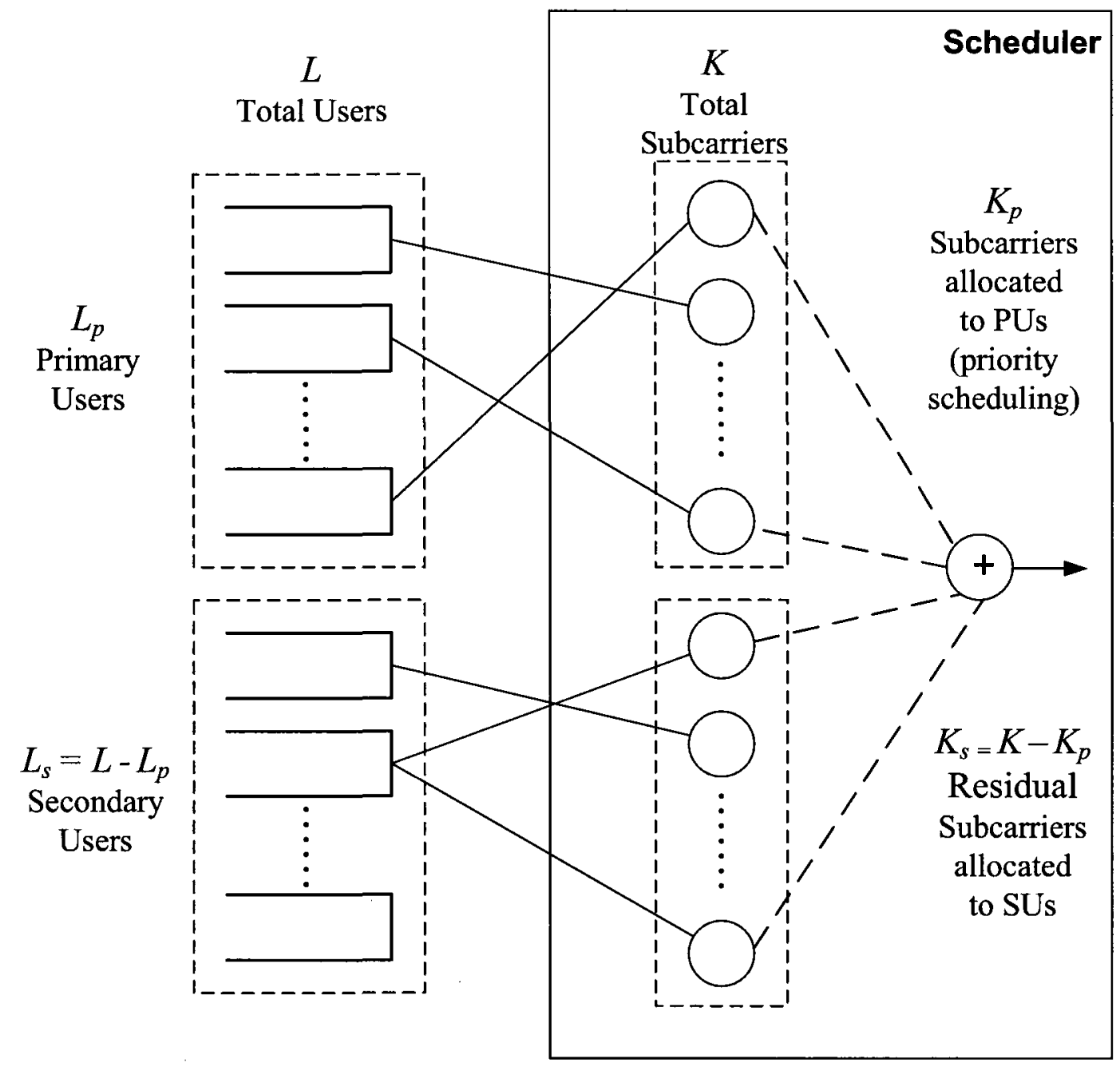

Figure 2.9: Model for joint PU-SU RRM at the BS

PU-SU RRM model. Considering the model in Fig. 2.9, the $L$ buffers on the left indicate the user, and the $K$ servers on the right indicate resources available at the BS and are similar to the notation used in [25]. The $K$ resources are a generic quantity and could be in the form of time slots, frequency bands or codes, and in this thesis as shown in Fig. 2.9, the $K$ resources are considered as the $K$ subcarriers available in one frame for an OFDM system. 
The total $L$ users are divided into $L_{p}$ PUs and $L_{s}=L-L_{p}$ SUs as shown in Fig. 2.9, where $K, L, L_{p}$ and $L_{s}$ are positive integers. In any time slot, according to the resource allocation policy, any of the $K_{p} \leq K$ subcarriers are assigned to the PUs. Since providing subscribed PUs with a satisfactory QoS is the primary goal of the RRM model, the SUs will be allocated $K_{s}=K-K_{p}$ subcarriers if and only if $K_{p}<K$, where $K_{p}$ and $K_{s}$ are positive integers.

Therefore, in order to give priority to PUs, the subcarriers will need to be assigned to the PUs first ensuring a satisfactory QoS for the PUs. After this initial assignment, the remaining subcarriers can then be given to the SUs, and hence providing a QoS guarantee for the SUs may not always be possible. The SUs may obtain a good QoS when the PU demand is low, but cannot be guaranteed QoS when the PU demand is high. Resource allocation in each of the two levels of subcarrier assignment first for the PUs and then for the SUs could be performed based on scheduling policies described in $[25,26]$ and references therein.

Therefore according to the proposed framework and the RRM model, it can be concurred that the SUs cannot be guaranteed QoS at all times, and hence the SU access would be recommended for Non Real Time (NRT) delay insensitive application classes. These NRT application classes support applications such as data-download, web browsing, email, instant messaging etc. which are independent of the delay in the system.

A special provisioning for guaranteed QoS may be provided to SUs attempting a 911 emergency call. Spectrum reserved for overload protection may be utilized in such a case for these SUs.

Providing equal status in scheduling of the PUs and SUs at the BS could be another way for joint RRM at the BS. However, the proposed system framework is 
based on provisioning of $\mathrm{SU}$ access without deterioration to the PU service, due to the limited spectrum available at the BS. Hence the RRM at the BS based on this status approach may be difficult to realize, even though the pricing model provides SU admission control as will be detailed in Chapter 4 .

\subsection{Summary}

The key aspects from this chapter are summarized as follows:

- Simple and practical all-IP based distributed framework for providing temporary wireless access to SUs as well as additional profits to WSPs from SU access is proposed.

- The proposed distributed framework is based on the idea that allows orthogonal coexistence of PUs and the SUs to function at the same BS in the same spectrum band licensed by the WSP, assuming efficient RRM at the BS.

- Signaling framework for allowing SU-BS interaction for unsubscribed SU access has been proposed.

- SU identification using the DSCP byte from the IP header is also proposed.

- Joint PU-SU RRM model for providing SU access without deterioration to the PU service is also described in this chapter.

The critical decision at the SU terminal to select best possible network (BS) for obtaining temporary access is based on the network (BS) selection criterion and is described in the next chapter. 


\section{Chapter 3}

\section{Network Selection and Price-based Handoff}

In current infrastructure based networks, WSPs provide subscribers (PUs) with wireless access and a guaranteed QoS as and when required. With the emergence of the DSA paradigm, temporary wireless access could be made available to SUs on an opportunistic basis as described in the previous chapters. With respect to the proposed framework, spectrum information transmitted by the BSs of WSPs over the broadcast channel F-SUBCH is available to the SUs in the coverage area of the transmitting BSs as shown in Fig. 2.3 and Fig. 2.4 from Chapter 2. However, the key decision to be made at the SU terminal is that "Which BS is the most appropriate for obtaining temporary wireless access?"

It must be noted that, multiple BSs with multiple RATs belonging to the same WSP could possibly transmit their local spectrum information over their F-SUBCH to the SUs in the coverage area. Since the spectrum utilization characteristics are BS specific, the SUs need to select their appropriate BS and consequently RAT available at the BS and the WSP that the BS belongs to for SU temporary wireless access, thus making this framework distributed.

The BS selection criterion at the SU terminal proposed in this chapter is designed to selects a BS autonomously without SU intervention. The term BS selection is interchangeably used with the term network selection throughout this thesis.

This chapter proposes a simple generalized criterion for autonomous network 
(BS) selection at the SU terminal. This criterion allows the SUs to select the network (BS) on-demand according to their service requirements, thus making this criterion SU-centric. Also considering the fact that, other BSs (of same or different WSPs) might be able to offer wireless access to the SUs for a significantly lower price than the current $\mathrm{BS}$ at a later time due to variable $\mathrm{PU}$ demand, a scheme for a potential price-based handoff to another BS is proposed. The primary feature of this chapter is that, the network selection and price-based handoff scheme are initiated and operated autonomously at the SU terminal, without the need for user intervention.

The organization of this chapter is follows: A brief literature review is initially presented in Section 3.1. The basic criterion for network (BS) selection at the SU terminal is described in Section 3.2, while a generalized version of this criterion for network (BS) selection incorporating the SU terminal capabilities and simultaneous requirement of multiple application classes by the $\mathrm{SU}$ is elaborated in Section 3.3. A SU terminal initiated price-based handoff scheme is then presented in Section 3.4, and the key contributions from this chapter are summarized in Section 3.5.

\subsection{Literature Review}

RAT selection under Common Radio Resource Management (CRRM) framework in heterogeneous networks has been studied in $[27,28]$. The concept explored in $[27,28]$ offers an approach for efficient joint management of multiple RATs, where a single WSP may have different RATs with different capacities, cell sizes and services supported. A metric based selection, where the WSPs assign the appropriate RAT to best serve service requirements of the subscribed user, while taking into account the operational efficiency at the WSP has been the focus in $[27,28]$.

Although the RAT is selected to appropriately suit the requirements of a sub- 
scriber in $[27,28]$, the basic structure is based on assignment of a particular RAT by the WSP and hence this type of selection can be classified as network (WSP) centric.

On the other hand, [29] described a process for automated network selection in heterogeneous networks with a user-centric perspective, where the user terminal selects the appropriate network from a set of heterogeneous networks available in the area. Even though the work in [29] presents a variety of attributes that the network provides the user terminal before selection and also defines building blocks for such a kind of infrastructure, the mechanism for providing spectrum information to the user terminals has not been addressed in [29].

A parallel work in the area of cognitive networks aiming to provide mechanism for transmitting spectrum information for network selection to the SUs based on a centralized architecture was addressed in [18]. Various schemes using the CPC manager (one per region) to collect spectrum information from the WSPs and transferring it to the SUs over the CPC were described in [18]. However, a criterion for selection of a particular WSP based on the spectrum information is not clearly described in [18]. Chapter 2 in this thesis proposes a framework for allowing SUs to gain spectrum information from the BSs of the WSPs in a distributed manner.

The focus of this chapter is to develop a simple and generalized criterion for autonomous network (BS) selection and a price-based handoff scheme at the SU terminal, which could be applicable to heterogeneous networks and cognitive networks.

\subsection{Basic Criterion for Autonomous BS Selection at the SU Terminal}

As described in the previous chapters, the distributed nature of the proposed framework requires the BSs of every WSP to broadcast their spectrum information to the SUs in the area, since the spectrum information is BS specific and related 
to the residual spectrum at that particular BS. From the SUs' perspective, the key information broadcasted over the F-SUBCH by every BS is the price charged by the WSPs to the SUs for temporary wireless access and the application classes offered by the BSs. Detection of the F-SUBCH transmitted from a particular BS also allows the SUs to gauge the signal strength of the broadcasted signal.

The criterion for network (BS) selection at the SU terminal is based on the following factors:

- Application class requirements of the SU

- Price per application class to be paid by the SUs for temporary wireless access

- Signal strength, i.e. the Signal to Noise Ratio (SNR) observed at the SU terminal

- RATs supported by the BS of a WSP

Before proceeding further, the notations relevant to this chapter are defined as follows:

- $\mathrm{BS}_{i}$, where $i \in\{1,2, \ldots, w\}$ denotes the index for the $\mathrm{BS}$ considering that the $\mathrm{SU}$ is located in the coverage area of $w$ BSs.

- $s_{i, j}$ denotes the price charged by $\mathrm{BS}_{i}$ to the SUs for temporary wireless access with application class $j$. This SU price $s_{i, j}$ is defined for a finite block of $B$ bytes with the unit ' $\$ / B$ ' for NRT delay insensitive applications. In case of RT delay sensitive applications $s_{i, j}$ is defined for a fixed time interval $t$ seconds with the unit ' $\$ / t$ '. It must be noted that the monetary unit considered throughout this thesis is dollars denoted by ' $\$$ '. 
- $\gamma_{i}$ represents the SNR detected by the SU on scanning the F-SUBCH transmitted from the $\mathrm{BS}_{i}$, such that $\gamma_{i}$ is a positive real number measured in $\mathrm{dB}$.

- $\eta_{i}$ represents the average achievable link spectral efficiency based on the SNR $\gamma_{i}$ for the SU-BS ${ }_{i}$ link, where $\eta_{i}$ is a positive real number with the unit b/s/Hz.

- $A_{o, i}=\{j: j \in \mathbb{N}, j \leq m\}$ denotes the set of application classes offered by $\mathrm{BS}_{i}$ to the SUs, where $j$ is the index for the application class and the maximum number of application classes offered by the BS of a WSP are assumed to be positive integer $m . \mathbb{N}$ indicates the set of natural numbers.

- $A_{r}=\{j: j \in \mathbb{N}, j \leq n\}$ denotes the set of application classes requested by the $\mathrm{SU}$ for temporary wireless access, where the maximum number of application classes that can be requested is limited by the positive integer $n$.

- $B_{s l}=\left\{B S_{i}: s_{i, j}=\min \left(\sum_{j \in A_{r}, j=1}^{n} s_{1, j}, \sum_{j \in A_{r}, j=1}^{n} s_{2, j}, \ldots, \sum_{j \in A_{r}, j=1}^{n} s_{w, j}\right)\right\}$ denotes the short list of BSs, which provide the temporary wireless access for application classes requested by the $\mathrm{SU}\left(A_{r}\right)$ at the same minimum $\mathrm{SU}$ price.

Figure 3.1 illustrated the critical information obtained by any SU terminal on scanning and detecting the F-SUBCH from the BSs of multiple WSPs. Three WSPs have been considered in Fig. 3.1 to be consistent with the basic scenario depicted in Fig. 2.3 from Chapter 2, where a $\mathrm{SU}$ is situated in the coverage area of three WSPs with different RATs.

The potential QoS that could be achieved by the SU depends on the average SNR $\left(\gamma_{i}\right)$ of the F-SUBCH transmitted from the $\mathrm{BS}_{i}$. However, throughout this work, average achievable link spectral efficiency based on the SNR is used in all the 


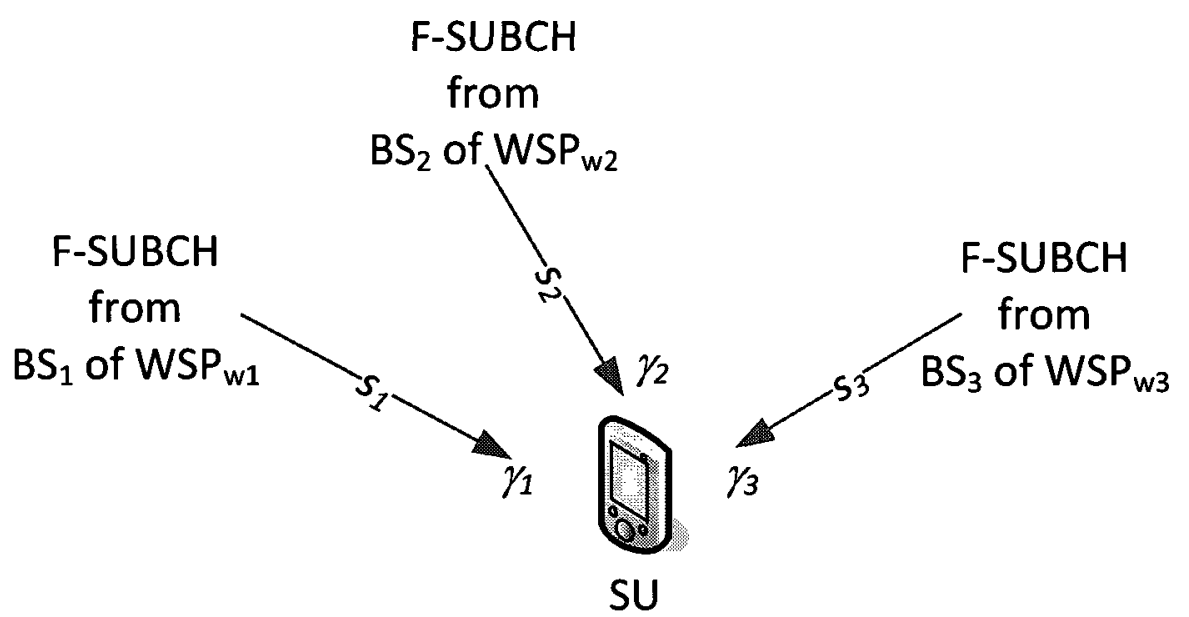

Figure 3.1: SU terminal obtaining spectrum information from multiple BSs

analysis, as it offers a better intuition with respect to the achievable data-rates for the SUs in the system. Considering a constant Bit Error Rate (BER) requirement based on the application class requirements, the average achievable link spectral efficiency for the $\mathrm{SU}_{-} \mathrm{BS}_{i}$ link can be defined as follows [30]:

$$
\eta_{i}=\log _{2}\left(1+\frac{-1.5 \gamma_{i}}{\ln \left(5 B E R_{j}\right)}\right) \text {, }
$$

where $B E R_{j}$ is the BER required for application class $j$.

\subsubsection{Rationale behind the Network Selection Criterion}

Without loss of generality, from the SUs' perspective, the selection of a network for obtaining temporary wireless access needs to satisfy the following attributes:

- Least SU price among the prices charged by the other WSPs in the coverage area. 
- Good signal strength (good SNR)

Considering the SU service contract and joint PU-SU RRM structure defined in Chapter 2, no guarantee for QoS can be made by the WSPs to the SUs, therefore the SUs will aim to select the network with the least price and the best possible spectral efficiency. Also as described briefly in Chapter 1 and elaborated in depth in Chapter 4, the price paid by the SUs is inversely proportional to the residual spectrum available at the BS for SU temporary wireless access. Therefore, the larger the residual spectrum that is available at the BS for $\mathrm{SU}$ access, the lower the $\mathrm{SU}$ price. This also implies that, connecting to the BS of a WSP with the least SU price and best possible spectral efficiency can potentially translate into better data-rate after the SU has been accepted into the network of the selected BS.

\subsubsection{BS Selection Criterion for Single Application Class}

Considering a single application class requirement at the SU terminal, the basic criterion for autonomous network (BS) selection at the SU terminal is given as

$$
i^{*}= \begin{cases}\arg \min _{i=1,2, \ldots, w}\left(s_{i, j}\right), & \text { if } \exists ! i^{*}, \\ \arg \max _{i \in B_{s l}}\left(\eta_{i}\right) & \text { otherwise. }\end{cases}
$$

The above function selects the $\mathrm{BS}_{i}^{*}$ such that it provides wireless access with the least price among other BSs in the coverage area. However, if multiple BSs offer the wireless access at the same minimum price, then the BS with the maximum spectral efficiency is selected from among the shortlist. It must be noted that, the application class $j$ requested by the $\mathrm{SU}$ is assumed to be offered by all the BSs, i.e., $A_{r} \subset A_{o, i}$.

For example, consider the SU prices for a NRT application class (web browsing) transmitted from the three BSs as $s_{1, j}=\$ 1 / \mathrm{MB}, s_{2, j}=\$ 1.5 / \mathrm{MB}$ and $s_{3, j}=$ 
$\$ 1.2 / \mathrm{MB}$, where $\mathrm{MB}$ denotes Megabytes of data transfer, and the average achievable link spectral efficiencies calculated at the SU terminal are $\eta_{1}=2 \mathrm{~b} / \mathrm{s} / \mathrm{Hz}, \eta_{2}=3$ $\mathrm{b} / \mathrm{s} / \mathrm{Hz}$ and $\eta_{3}=4 \mathrm{~b} / \mathrm{s} / \mathrm{Hz}$. The SU terminal will then select $\mathrm{BS}_{1}$, since it had quoted the lowest SU price for providing temporary wireless access for the web browsing application. In this case, there is a BS with the unique lowest SU price, and hence the network selection did not involve the spectral efficiency at the SU terminal as the SUs are not guaranteed any QoS, and the data-rate received by the SU would thus depend on the spectrum utilization at the BS.

However, if the SU prices in the above example are slightly modified as follows $s_{1, j}=\$ 1 / M B, s_{2, j}=\$ 1 / M B$ and $s_{3, j}=\$ 1.2 / M B$ with $\mathrm{BS}_{1}$ and $\mathrm{BS}_{2}$ both quoting the same lowest prices, then instead of making a random selection based on the SU price, the SU terminal selects the BS with the better spectral efficiency, i.e., in this case $\mathrm{BS}_{2}$.

Following network (BS) selection, the SU will need to send a spectrum access request 'SAReq' to the selected $\mathrm{BS}_{i}^{*}$ over the R-SUACH before they can obtain temporary wireless access, as described in Chapter 2 .

Although the data-rate needs be considered as the defining factor in selecting a BS for wireless access, it must be noted that the SU terminals cannot estimate the data-rate before connection to WSPs only on the basis of the SNR from the detected broadcast signal, i.e., the F-SUBCH. The other reason for using the spectral efficiency in the criterion for network selection is that, the SUs cannot be guaranteed a specific data-rate due to the varying demand of the subscribed PUs and the proposed framework is aimed at providing $\mathrm{SU}$ access without affecting the operation of the PU service. It must be noted that the spectral efficiency calculated on the SU terminal is the estimated spectral efficiency based on the SNR observed at the SU terminal 
from $\mathrm{BS}_{i}$.

It must be stressed that the SUs are allocated the leftover resources at the BS, and hence in general the NRT delay insensitive application classes are recommended for SU access, but RT application classes can also be supported in periods of low PU demand. However, assuming that a SU requires temporary wireless access for web browsing as well as is interested in downloading a file using FTP (File Transfer Protocol) simultaneously, the SU terminal must select a BS which offers both application classes. Therefore, the above BS selection criterion needs to be extended for accommodating network (BS) selection involving multiple application class requirements of the SUs.

\subsection{Generalized BS Selection Criterion at the SU terminal}

This subsection describes the generalized criterion for BS selection and incorporates the following aspects:

- SU requirement for simultaneous use of multiple application classes

- RATs supported on the SU terminal

Although, the SU terminals are assumed to support any RAT, the following generalized network selection criterion also considers SU terminals, which may have support only to a limited number of RATs. But these SU terminals still need to have the capability for spectrum scanning and reconfiguration to the RAT provided by the BSs of the WSPs. The additional notations specific to this generalized formulation are given below:

- $\mathcal{R}_{\mathcal{B}}$ denotes the set of RATs $x$ supported by the $\mathrm{BS}_{i}$, where $x$ is the index of the RAT. 
- $\mathcal{R}_{\mathcal{S}}$ denotes the set of RATs $x$ supported by the SU terminal.

- $I_{i, j}$ denotes a binary integer variable for checking the availability of the $j^{\text {th }}$ application class requested by the $\mathrm{SU}$ with those offered by the $i^{\text {th }} \mathrm{BS}$.

- $B_{i, x}$ denotes a binary integer variable for verifying the support for RAT $x$ provided by $\mathrm{BS}_{i}$ at the $\mathrm{SU}$ terminal.

A positive integer $M$ assumed to have a very large value, e.g., $M \geq 1000$, is assigned to $\mathrm{SU}$ price whenever the application class required by the $\mathrm{SU}$ is not offered by the $\mathrm{BS}_{i}$ and can be given as follows:

$$
s_{i, j}=M \text { if } j \in A_{r} \text {, but } j \notin A_{r} \bigcap A_{o, i} .
$$

Therefore, the generalized network (BS) selection criterion to be calculated at the $\mathrm{SU}$ terminal can be given as follows:

$$
i^{*}= \begin{cases}B_{i, x} \times\left(\prod_{j \in A_{r}, j=1}^{n} I_{i, j}\right) \times\left(\arg \min _{i=1,2, \ldots, w}\left(\sum_{j \in A_{r}, j=1}^{n} s_{i, j}\right)\right), & \text { if } \exists ! i^{*}, \\ B_{i, x} \times\left(\prod_{j \in A_{r}, j=1}^{n} I_{i, j}\right) \times\left(\arg \max _{i \in B_{s l}}\left(\eta_{i}\right)\right), & \text { otherwise }\end{cases}
$$

where

$$
\begin{gathered}
I_{i, j}= \begin{cases}1, & \text { if } j \in A_{r} \cap A_{o, i}, \\
0, & \text { otherwise }\end{cases} \\
B_{i, x}= \begin{cases}1, & \text { if } x \in \mathcal{R}_{\mathcal{B}} \cap \mathcal{R}_{\mathcal{S}}, \\
0, & \text { otherwise. }\end{cases}
\end{gathered}
$$

The binary integer variable $I_{i, j}$ ensures the selection of only those networks where all the application classes requested by the SU are offered by the BSs of the WSPs. 
The other binary integer variable $B_{i, x}$ in the objective function ensures that the RAT provided by the BS is supported by the $\mathrm{SU}$ terminal. The function in (3.4) is merely a generalized version of (3.2) and aims to minimize the sum price for all the application classes requested by the SU. Also in case of the sum price quoted by the BSs are to be equal, the SU terminal selects the BS with the best spectral efficiency from among the short listed BSs with the minimum price.

\subsection{Pricing based Handoff Initiated by the SU Terminal}

\subsubsection{Motivation}

As described previously, the SU price $s_{i, j}$ for NRT and RT applications is defined for a finite block of $B$ bytes and finite time $t$ seconds respectively. After the initially defined contract for $B$ block of bytes or $t$ seconds depending on the application class expires, the SU might still be interested in continuing with the service and would renew the service contract for the next $B$ blocks or $t$ seconds.

However, owing to the fluctuations in PU demand at the BS, the SU price may increase for the next $B$ blocks or $t$ seconds. Therefore instead of renewing the contract, the SU terminal could potentially search for other networks (BSs) in the area, who could be offer similar service for the same application class for a lesser price than the new SU price quoted by the current BS for next $B$ blocks or $t$ seconds. This possibility gives rise to a price-based handoff, assuming a regular handoff based on signal strength is not required. 


\subsubsection{Price-based Handoff Scheme}

The following example considers of a SU interested in acquiring temporary wireless access for 'web browsing'. Using the criterion for network selection in (3.4), the SU selects a particular $\mathrm{BS}_{i}$ of a WSP to obtain wireless access for a block of $B$ bytes of data at time instant $T_{1}$. Assuming that the data transfer of the $B$ bytes requires $T_{2}-T_{1}$ seconds, at time instant $T_{2}$ the temporary service contract for $B$ bytes from $\mathrm{BS}_{i}$ expires and the new $\mathrm{SU}$ price $s_{i, j}\left(T_{2}\right)$ is calculated considering the new PU demand at the $\mathrm{BS}_{i}$.

The SU thus will have to pay the new price $s_{i, j}\left(T_{2}\right)$ for the next $B$ bytes or could handoff to another BS offering a lower SU price. Once a price based handoff is triggered based on the price-based handoff scheme in (3.5), the SU terminal will proceed to scan the F-SUBCH from all the BSs in the area, and then using the network selection criterion in (3.4) select the most suitable BS. The information related to SU price at time instant $T_{2}$ needs to be provided by the current $\mathrm{BS}_{i}$ well in advance for the SU to trigger a price-based handoff and proceed with network selection, thus continuing the service with minimum interruption.

Once the handoff is completed, the SU terminal will be terminated from the current $\mathrm{BS}$ and there could be a possible interruption to the SU service, due to authentication to the new BS and the subsequent mapping of the SUs' information. However, if the network (BS) selection criterion selects the same BS of the same WSP, then the handoff is avoided and the $\mathrm{SU}$ contract is renewed by the $\mathrm{BS}_{i}$ for the next $B$ block of bytes.

The price-based handoff will be triggered, if the ratio of the difference between the new price $s_{i, j}\left(T_{2}\right)$ and the old price $s_{i, j}\left(T_{1}\right)$ with respect to the the old price $s_{i, j}\left(T_{1}\right)$ is greater than $\mathrm{SU}$ defined cost threshold denoted by $C_{t h}$. 
The condition for price-based handoff can be given as

$$
\frac{s_{i, j}\left(T_{2}\right)-s_{i, j}\left(T_{1}\right)}{s_{i, j}\left(T_{1}\right)} \geq C_{t h},
$$

where $C_{t h}$ is a non-negative real number. The value of this cost threshold $C_{t h}$ is the only other parameter that needs to be defined by the SUs apart from the application classes required, before network selection. The parameter $C_{t h}$ offers allows for some amount of hysteresis before the price based handoff can occur triggering the network scanning and selection process.

For example, setting $C_{t h}=0.2$, triggers a price based handoff if there is a $20 \%$ increase in $s_{i, j}$. But, if $C_{t h}=0$, a price-based handoff will be triggered even if the $\mathrm{SU}$ price at time instant $T_{2}$ is slightly greater than the $\mathrm{SU}$ price at time instant $T_{1}$.

However, after the scanning the F-SUBCH from multiple BSs and observing the SU prices, the lowest SU price for the next time window may be very high (e.g. 2 or 3 times, the SU price paid by the SU for the previous time window) and not affordable to the SU. In such a case, the SU needs to have the option to terminate the service without handoff and reconnect at a later time. In order to allow the SU to terminate the connection beyond a certain rise in the SU price (for the next time window), the SUs need to set cost boundary denoted as $C_{b d}$. This cost boundary automatically disconnects the connection, if the SU prices for the next time window rise above the limit which is not affordable to the SU. Thus this cost boundary parameter is a SU defined parameter and is a positive real number such that $C_{b d}>C_{t h}$.

Since there can be a possibility for the SU to be in the coverage area of two BSs under the same WSP or BSs with two different RATs under the same WSP, the price-based handoff would increase in time and complexity in the following order:

- same RAT, same WSP, but different BSs (horizontal handoff) 
- different RAT, but same WSP (vertical handoff)

- same RAT, but different WSP

- different RAT, and different WSP.

\subsection{Summary}

The functionality and operation of the SU terminal within the distributed framework proposed in this thesis has been the primary focus of this chapter.

The key features in this chapter are listed below:

- A SU centric criterion for network selection, where the most suitable BS is selected by the SU for temporary wireless access.

- A SU terminal initiated price based handoff scheme is also proposed in this chapter, under the assumption that a normal handoff based on signal strength is not required.

- The autonomous operation of the SU terminal for network (BS) selection and price-based handoff required only two basic inputs from the SUs as listed below:

- The application classes required by the SU $\left(A_{r}\right)$

- The cost threshold beyond which the price-based handoff will be triggered $\left(C_{t h}\right)$

- the cost boundary beyond which the SU cannot afford to be connected and will prefer to disconnect the connection $\left(C_{b d}\right)$

The next chapter delves into the incentive based pricing model for charging SUs. 


\section{Chapter 4}

\section{Incentive based Pricing Model for Charging SUs}

\subsection{Introduction}

Developing a system framework for enabling SU access has been the primary motivation behind the work in this thesis. However, even though of the technology can be made available for enabling such temporary wireless access, it is the pricing strategy for charging the SUs that will dictate the successful implementation of any DSA framework. The pricing model described in this chapter is aimed at providing a separate pricing structure for the SUs taking into account the PU demand at the $\mathrm{BS}$ and the total spectrum utilization at the BS.

The price paid by the PUs (for the $t$ seconds or $B$ bytes depending on the application class) is used in the pricing model as the reference price for SU pricing, and provides a fair baseline for setting the SU price. The pricing model thus offers discounted prices or incentives for the SUs to obtain temporary wireless access in periods of low PU demand at the BS of a WSP. The rationale for the incentive based pricing model, where the PU demand dictates the SU pricing is illustrated in Fig. 4.1, and shows a basic demand curve based on the principles from microeconomics. The residual spectrum at the BS is plotted with respect to the price charged to the SUs.

Observing Fig. 4.1, the total PU demand at the $\mathrm{BS}_{i}$, which is dependent on time 


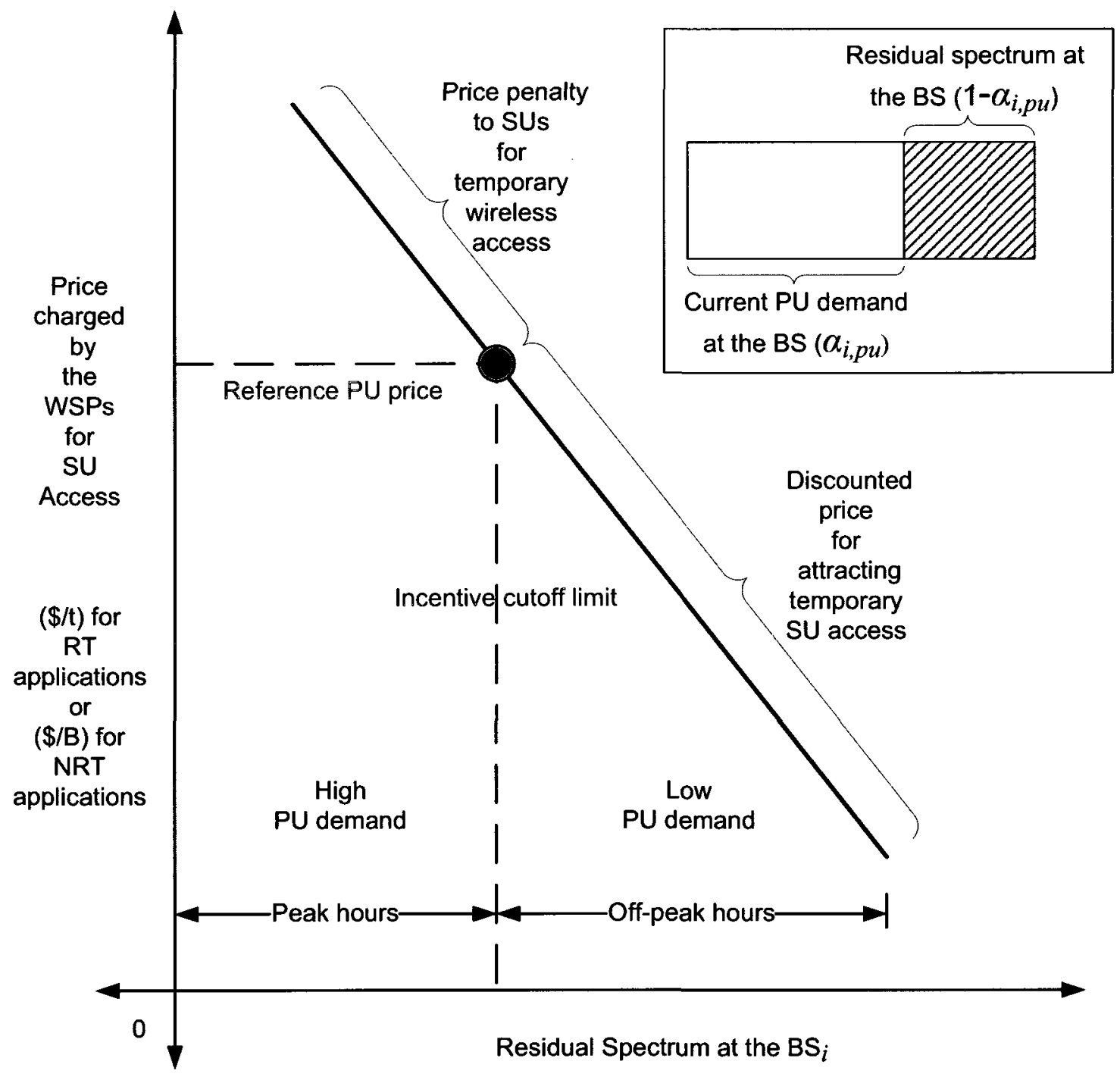

Figure 4.1: Demand curve and incentive based pricing

of the day and location of $\mathrm{BS}_{i}$ is denoted by $\alpha_{i, p u}$. Assuming the total spectrum at the $\mathrm{BS}_{i}$ to be 1, the residual part of the spectrum available for $\mathrm{SU}$ access is denoted as $1-\alpha_{i, p u}$. The SU price can be seen to increases as the PU demand at the BS increases, i.e., when the SU supply (residual spectrum at the BS) decreases. Therefore, it can be inferred from Fig. 4.1 that, the SU price is directly proportional the the PU demand and inversely proportional to the SU supply (residual spectrum at the BS). 
The notion of incentives can be defined based on the incentive cutoff limit, which is the point at which the reference price charged to the PUs is equal to the $\mathrm{SU}$ price. Beyond the incentive cutoff limit the price charged to the SUs rises above the reference PU price, thus discouraging the SUs from gaining wireless access during periods of high $\mathrm{PU}$ demand at the BS.

Since the SU price depends upon the PU demand, the PU price and the total spectrum utilization at the BS (PUs and SUs), every new SU entering the system is charged a higher SU price, thus giving the pricing model its opportunistic and dynamic structure. The proposed SU pricing model in this chapter will also be seen to inherent $\mathrm{SU}$ admission control, thus discouraging $\mathrm{SU}$ access as the amount of residual spectrum at the BS decreases. It must be noted that the curve shown in Fig. 4.1 is for illustrative purposes and the goal of this chapter is to define the curve.

Although the SUs may pay lower prices for the temporary spectrum access in comparison to PUs, it must be noted that the SUs are provided wireless access only when residual spectrum is available at the BS, and hence may not be guaranteed QoS. The pricing model thus operates in conjunction with the joint PU-SU RRM model enabling SU access along with PUs at the same BS, as detailed previously in Chapter 2.

\subsection{Literature Review}

Pricing for SUs in different system frameworks has been addressed before in the literature. For example, pricing SUs based on resource and power was proposed in [20], where the spectrum was directly shared with the SUs. However, this spectrum is leased by the WSPs through a central spectrum clearinghouse where the spectrum is pooled. Thus depending on the SU demand at the WSP, spectrum is borrowed 
from the centralized spectrum clearinghouse by the WSPs [20]. This approach where the SU price is based on the power or resource allocated could lead to concerns of tractability from the WSPs' and SUs' perspective with regards to the practical implementation of such a model.

In another example, a centralized Spectrum Policy Server (SPS) receives the SU requests for wireless access [19] as shown in Fig. 4.2 taken from [19]. An iterative bidding process takes place between the WSPs to serve the SUs, and this process is supervised by the SPS. The WSP with the best bid is then communicated to the $\mathrm{SU}$, who can then possibly accept or reject the bid. In this example as well, a centralized SPS negotiates with the WSPs on behalf of the SUs. The price charged to the SUs in this framework is demand responsive and based on the number of SUs requesting wireless access. This SPS helps in negotiating the prices, but the complexity and tractability of this framework from the implementation perspective could be prohibitive. In both the above examples, SU pricing is predominantly based on the SU demand, and a centralized architecture.

In terms of implementation of any pricing model, the centralized architecture in these two approaches requires all the WSPs to cooperate in deciding the prices, since the prices are based on the SU demand and the spectrum is pooled at a centralized location.

On the other hand, the proposed distributed framework is based on individual BSs selling their spectrum directly to the SUs. The SU pricing as explained in the introduction, is thus based on the PU demand at the BS, instead of the SU demand [19] or power [20]. The key feature of the proposed pricing model is that it provides a sense of price comparison to the SUs on the fair criteria of PU pricing and PU demand. The pricing model described in this chapter will be seen to depend on 


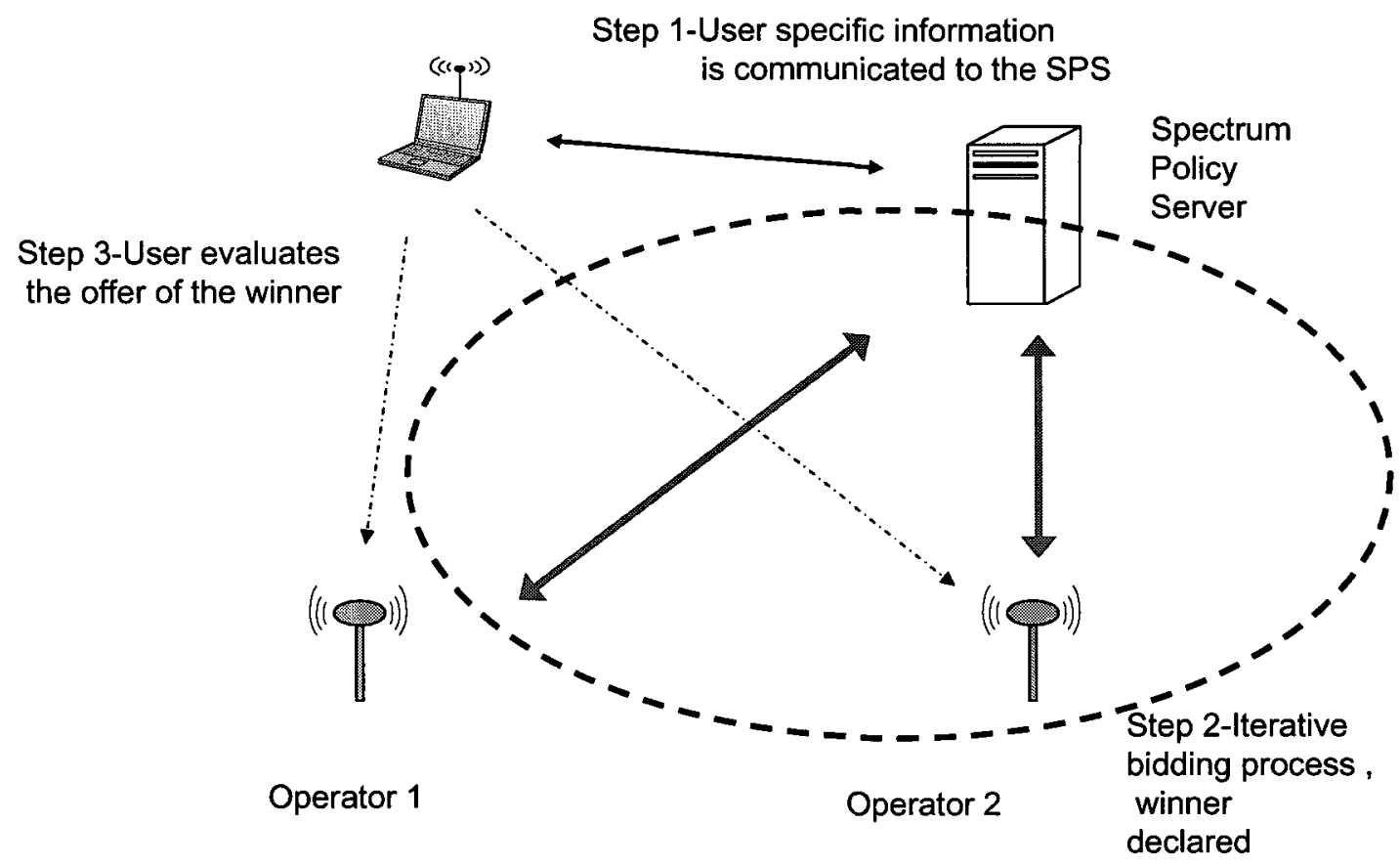

Figure 4.2: Pricing mechanism with spectrum policy server

the PU demand, the total spectrum utilization (PUs and SUs) at the BS and the PU price, and provide the WSPs with a variety of tuning parameters for adjusting the SU price.

\subsection{Incentive based Pricing Model for Secondary Users}

As described in the introduction, the SUs have a monetary incentive (with respect to the reference PU price) to obtain access to the radio spectrum when PU demand is low, while have a monetary penalty when PU demand is high. This reference PU price is denoted by $p_{i, j}$ and is the price charged by the $\mathrm{BS}_{i}$ of a WSP to PUs for wireless access with guaranteed QoS for application class $j$. This PU price is assumed to have the same unit as that of the SU price for fair comparison, i.e., $\$ / t$ for RT delay sensitive application classes and $\$ / B$ for NRT delay insensitive application classes. 
In this section, a tractable and implementable pricing model, with an inherent property for admission control of SUs at the BS is presented. This novel incentive based pricing model relates the total spectrum utilization at the BS, PU demand and fixed reference PU price to establish the SU price.

\subsubsection{Parameters and Terminology}

As the spectrum utilization characteristics at the BS have been observed to change with time and location [1], the Spectrum Utilization Factor (SUF) at the $\mathrm{BS}_{i}$ denoted by $\alpha_{i, t}$ is used to capture the dynamics of this variable utilization, where $\alpha_{i, t} \in[0,1]$. The SUF can be defined as the total spectrum utilization at the $\mathrm{BS}_{i}$, which depends on the total number of users (PUs and SUs) currently being served at the BS, i.e., $\alpha_{i, t}=\alpha_{i, p u}+\alpha_{i, s u}$, where $\alpha_{i, p u}$ and $\alpha_{i, s u}$ represent the part of the spectrum at $\mathrm{BS}_{i}$ occupied by $\mathrm{PUs}$ and $\mathrm{SUs}$ respectively and $\alpha_{i, p u}, \alpha_{i, s u} \in[0,1]$. Thus, the SUF value $\alpha_{i, t}=0$ indicates no utilization, while $\alpha_{i, t}=1$ indicates complete utilization of the spectrum at $\mathrm{BS}_{i}$.

Since every BS needs to reserve a certain part of its spectrum for handoff and overload protection, the amount of spectrum to be reserved is a BS parameter and is denoted as $\alpha_{i, h}$. Thus the total amount of spectrum available at $\mathrm{BS}_{i}$ for PUs or SUs is limited to the spectrum usage threshold and is denoted as $\alpha_{i, t h}=1-\alpha_{i, h}$, where $\alpha_{i, t h}, \alpha_{i, h} \in[0,1]$. However, it must be noted that as the SUs are not required to be guaranteed any QoS according to the RRM model in Chapter 2, the amount of spectrum that will be available for $\mathrm{SUs}_{s}$ at $\mathrm{BS}_{i}$ can be given as

$$
\begin{aligned}
\alpha_{i, s u} & =\alpha_{i, t h}-\alpha_{i, p u}, \\
& =1-\alpha_{i, h}-\alpha_{i, p u} .
\end{aligned}
$$

Equation (4.1) indicates that same principle as described in the RRM model from 
Chapter 2, that the SUs can be provided access to the residual spectrum if it exists at $\mathrm{BS}_{i}$, i.e., $\alpha_{i, p u}<\alpha_{i, t h}$.

The pricing model that will be presented next also internally handles admission of SUs by dynamically modulating the prices with respect to the fixed reference PU price and based on the PU demand. This SU admission control is achieved by penalizing the SUs on the price as the spectrum utilization $\alpha_{i, t}$ goes beyond a particular limit called the incentive cutoff limit set by $\mathrm{BS}_{i}$ and is denoted as $\alpha_{i, i c}$ where $\alpha_{i, i c} \in[0,1]$. This limit is called so, since the SUs have a monetary incentive $\left(s_{i, j}<p_{i, j}\right)$ to obtain wireless access for application class $j$ when $\alpha_{i, t}<\alpha_{i, i c}$. Therefore, as the spectrum utilization goes beyond the incentive cutoff limit, i.e., $\alpha_{i, t} \geq \alpha_{i, i c}$, the SUs need to pay more than the reference PU price $\left(s_{i, j} \geq p_{i, j}\right)$ to obtain the same wireless service for application class $j$, in turn discouraging the SUs to obtain temporary access.

It must be noted that $\alpha_{i, t h}$ and $\alpha_{i, i c}$ are the $B S$ configuration parameters and need to set for each $\mathrm{BS}_{i}$ by the WSP. The values chosen for these BS configuration parameters in this thesis are for illustration purposes and are based on intuition. However, in practice, it is extremely essential for the WSPs to select the most suitable BS configuration parameters not only from the profit and pricing perspective but also from the network functionality perspective. The following aspects related to network functionality may be considered before selection of these BS configuration parameters:

- The parameter $\alpha_{i, t h}$ defines the spectrum usage threshold and is based on the amount of spectrum reserved for handoff given by $\alpha_{i, h}$. This parameter $\alpha_{i, h}$ needs to set considering the PU usage behavior at various times during the day. Also sufficient overload protection may need to be provided to allow statistically new PUs to entering the BS at any given point in time. 
- Incumbent SUs with RT or near-RT applications may face possibility of termination due to the sudden influx of PUs in the system. Also SUs being handed off (regular handoff based on signal strength) from the neighboring BSs of the same WSP may also be using RT or near-RT applications. Thus overload protection may also be extended to provide these SUs with QoS for the promised 't' seconds, after which they may be handed off or terminated.

- The parameter $\alpha_{i, i c}$ is the incentive cutoff limit, and is primarily useful in attracting the SUs during periods of low PU demand. This parameter must be carefully selected considering the average $\mathrm{PU}$ usage at $\mathrm{BS}_{i}$ at various times of the day, while simultaneously generating profits to the WSPs by providing sufficient incentives for the SUs to acquire temporary wireless access.

A list of all the notations described above is shown as follows:

- $p_{i, j}$ : Fixed price charged by the $\mathrm{BS}_{i}$ of a WSP for application class $j$ to PUs for guaranteed wireless access.

- $s_{i, j}$ : Price charged by the $\mathrm{BS}_{i}$ of a WSP for application class $j$ to SUs for temporary wireless access, based on PU price $p_{i, j}$ and PU demand $\left(\alpha_{i, p u}\right)$ and the $\operatorname{SUF}\left(\alpha_{i, t}\right)$.

- $\alpha_{i, p u}$ : Spectrum at $\mathrm{BS}_{i}$ occupied by PUs, i.e., PU demand.

- $\alpha_{i, h}$ : Spectrum at $\mathrm{BS}_{i}$ reserved for handoff and overload protection.

- $\alpha_{i, t h}$ : Threshold for spectrum utilization; $\alpha_{i, t h}=1-\alpha_{i, h}$.

- $\alpha_{i, s u}$ : Spectrum at $\mathrm{BS}_{i}$ occupied by SUs, such that $\alpha_{i, s u}$ exists if and only if $\alpha_{i, p u}<\alpha_{i, t h}$. 
- $\alpha_{i, t}$ : Total utilization of spectrum at $\mathrm{BS}_{i}$, i.e., the SUF; $\alpha_{i, t}=\alpha_{i, p u}+\alpha_{i, s u}$.

- $\alpha_{i, i c}$ : Incentive cutoff limit beyond which $s_{i, j}>p_{i, j}$.

$\alpha_{i, p u}, \alpha_{i, h}, \alpha_{i, t h}, \alpha_{i, s u}, \alpha_{i, t}, \alpha_{i, i c} \in[0,1]$.

\subsubsection{Formulation for the Incentive based Pricing Model}

The basic formulation of the dynamic incentive based pricing model for charging SUs to gain temporary wireless access to application class $j$ is given as

$$
s_{i, j}=f_{i, j}\left(\alpha_{i, t}\right) \times p_{i, j}
$$

where $s_{i, j}, f_{i, j}\left(\alpha_{i, t}\right)$ and $p_{i, j}$ are non-negative real numbers. The term $f_{i, j}\left(\alpha_{i, t}\right)$ captures the variability of spectrum utilization at $\mathrm{BS}_{i}$, and can be considered as the normalized SU price for application class $j$ with respect to the reference fixed PU price $p_{i, j}$. This term is based on the log barrier function [31] and provides inherent SU admission control at $\mathrm{BS}_{i}$ such that

$$
\lim _{\alpha_{i, t} \rightarrow \alpha_{i, t h}} f_{i, j}\left(\alpha_{i, t}\right)=\infty
$$

thus helping restrict SU access as the SUF at the BS increases. The normalized SU price $f_{i, j}\left(\alpha_{i, t}\right)$ is defined as follows

$$
f_{i, j}\left(\alpha_{i, t}\right)= \begin{cases}-\ln \left(1-\left(\frac{\alpha_{i, t}}{\alpha_{i, t h}}\right)^{n_{i, j}}\right), & \text { if } 0 \leq \alpha_{i, t}<\alpha_{i, t h}, \\ \infty, & \text { if } \alpha_{i, t h} \leq \alpha_{i, t} \leq 1\end{cases}
$$

where $n_{i, j}$ is a positive real number representing the exponent in $f_{i, j}\left(\alpha_{i, t}\right)$ referred to as the Incentive Cutoff Factor (ICF).

As described previously, the two parameters $\alpha_{i, i c}$ and $\alpha_{i, t h}$ are the BS configuration parameters and need to be set at the BS by the WSP, while the SUF $\left(\alpha_{i, t}\right)$ is 


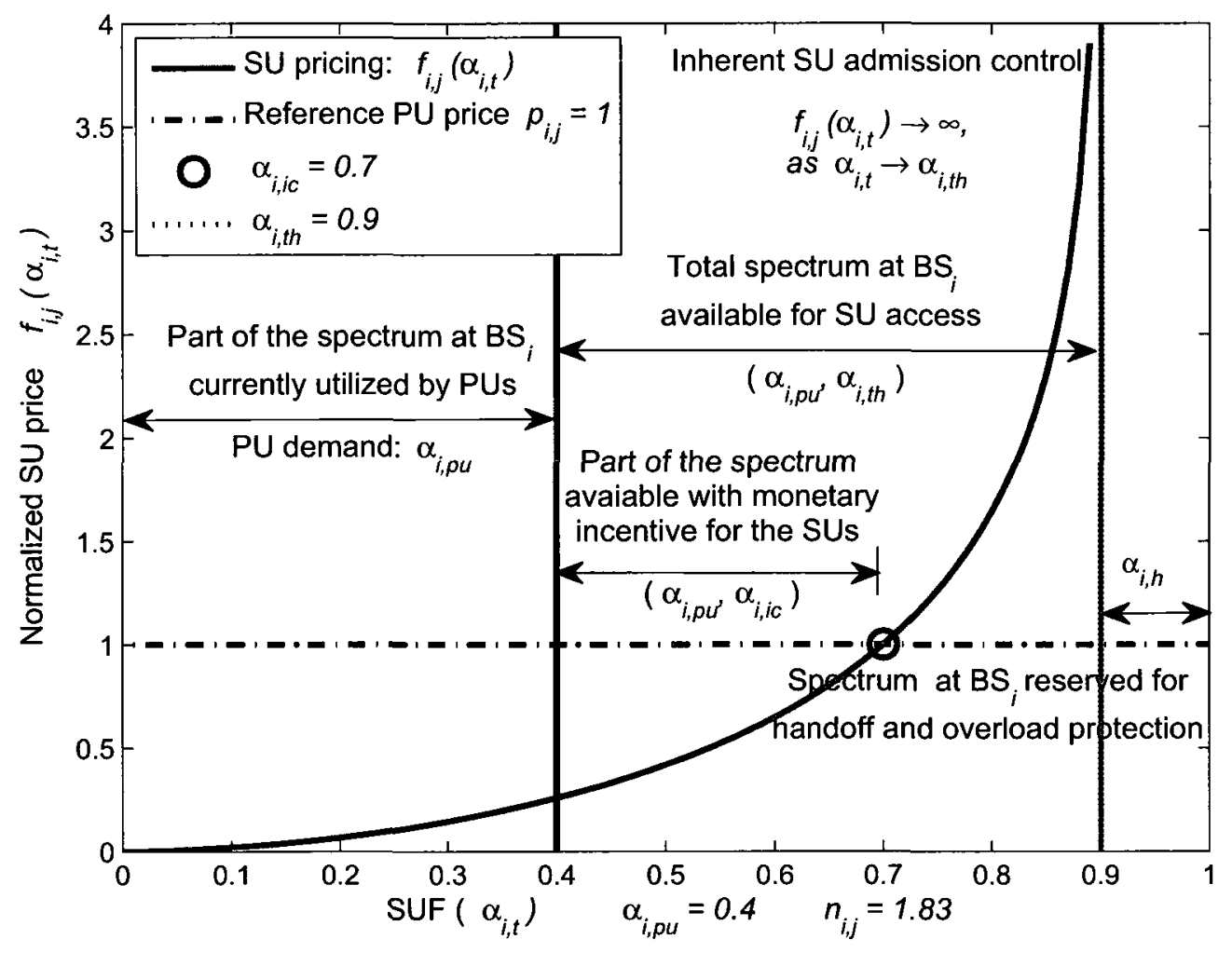

Figure 4.3: Variable nature of the incentive based SU pricing model

an external parameter and depends on the number of PUs and SUs being served by $\mathrm{BS}_{i}$. The ICF is useful in incorporating all these parameters together into the pricing model by solving (4.4) for $\alpha_{i, t}=\alpha_{i, i c}$ and $f_{i, j}\left(\alpha_{i, t}\right)=1$ as follows:

$$
n_{i, j}=\frac{\ln \left(1-e^{-f_{i, j}\left(\alpha_{i, t}\right)}\right)}{\ln \left(\alpha_{i, t}\right)-\ln \left(\alpha_{i, t h}\right)}=\frac{\ln \left(1-e^{-1}\right)}{\ln \left(\alpha_{i, i c}\right)-\ln \left(\alpha_{i, t h}\right)} .
$$

It can be seen that the ICF defines the basic SU price as a function of the configuration parameters set at $\mathrm{BS}_{i}$ and the $\mathrm{PU}$ price set for application class $j$. The ICF thus defines the division between monetary incentive and monetary penalty to the SUs.

Figure 4.3 illustrates the variable nature of $f_{i, j}\left(\alpha_{i, t}\right)$ and thus of SU price in relation to the SUF, the PU demand and fixed reference PU price $p_{i, j}=1$ with $\mathrm{BS}$ 
configurations parameters set as $\alpha_{i, t h}=0.9$ and $\alpha_{i, i c}=0.7$. The part of the spectrum available for $\mathrm{SU}$ access $\left(\alpha_{i, p u}, \alpha_{i, t h}\right)$ is also shown in Fig. 4.3 with a monetary incentive for the SUs when $\alpha_{i, t} \in\left(\alpha_{i, p u}, \alpha_{i, i c}\right)$, while a monetary penalty when $\alpha_{i, t} \in\left[\alpha_{i, i c}, \alpha_{i, t h}\right)$.

\subsubsection{Pricing Flexibility with Price Leveling Factor}

It can be seen in Fig. 4.3 that the SU price is very low for most part of spectrum and suddenly shoots towards infinity after $\alpha_{i, t}>\alpha_{i, i c}$. If a fixed cost of $c_{i, j}=0.5$ is assumed at $\mathrm{BS}_{i}$ for providing wireless access to SUs for application class $j$, then the WSPs will experience losses in providing wireless access to a few SUs, until $s_{i, j}>c_{i, j}$.

Thus, in addition to the variable nature of $s_{i, j}$, the WSPs need to have a mechanism for pricing flexibility to enable price adjustment for profit maximization and for competing with the prices set by the other WSPs in the region. To aid this purpose, the Price Leveling Factor (PLF) denoted by $m_{i, j}$, is introduced. This factor allows the adjustment in SU prices according to WSP requirements, keeping the configuration parameters $\alpha_{i, i c}$ and $\alpha_{i, t h}$ set by the WSP unchanged. Therefore the PLF is dependent on the dynamic SU price, the fixed reference PU price and the application class $j$ along with the configuration parameters.

The adjusted SU price $\bar{s}_{i}$ can be given as follows:

$$
s_{i, j}=\left(f_{i, j}\left(\alpha_{i, t}\right)\right)^{m_{i, j}} \times p_{i, j}
$$

where $m_{i, j}$ is the exponent of $f_{i, j}\left(\alpha_{i, t}\right)$ and is a non negative real number.

Considering the previous example, where the WSPs would experience losses if the SU price $s_{i, j}<c_{i, j}=0.5$, the $\mathrm{SU}$ price will need to be raised above the cost $c_{i, j}$ in order for the WSPs to make profit from each of the SUs. This PLF can thus be used by the WSPs to avoid losses by raising the SU price above the cost incurred in providing wireless access for SUs. 
The value of the PLF can thus be found by rearranging (4.6) and setting $\overline{s_{i, j}}$ equal to a certain incentive based price at a particular SUF $\left(\alpha_{i, t}\right)$. For example, $s_{i, j} \geq c_{i, j}$ at $\alpha_{i, t}=\alpha_{i, p u}$ means that the price charged to the first SU and the subsequent SUs will be equal to or greater than the WSP cost $\left(c_{i, j}\right)$, thus avoiding any losses to the WSPs. The next chapter provides an insight into the economic aspect for SU pricing, where the PLF will be shown to be useful in competing with the SU prices set by other WSPs in the area, while guaranteeing individual profit per SU to every WSP in the area.

The peculiar feature of PLF is that when $m_{i, j}=0$, the SU price model becomes static, i.e., $\bar{s}_{i, j}^{-}=p_{i, j}$. Thus as $m_{i, j}$ increases, $\overline{s_{i, j}}$ reduces in the interval of SU price incentive $\left(\alpha_{i, p u}, \alpha_{i, i c}\right)$, while $\bar{s}_{i, j}$ shoots up in the interval of SU price penalty $\left[\alpha_{i, i c}, \alpha_{i, t h}\right)$. Figure 4.4 illustrates the price adjustment feature introduced by the PLF and the effect of different values of PLF on the normalized SU price $f_{i, j}\left(\alpha_{i, t}\right)$ in the intervals of incentive and penalty. The configuration parameters used for Fig. 4.4 are the same as in Fig. 4.3, i.e., $\alpha_{i, t h}=0.9$ and $\alpha_{i, i c}=0.7$ and the normalized SU price $f_{i, j}\left(\alpha_{i, t}\right)$ is plotted with respect to a fixed reference PU price $p_{i, j}=1$. It can be also seen from Fig. 4.4 that the the pricing curves with $m_{i, j}=0.2$ and $m_{i, j}=0.6$ avoid the WSP from incurring losses from SU access considering the fixed cost $c_{i, j}=0.5$.

As observed from (4.6) and Fig. 4.4, the PLF controls the slope and hence variance of $f_{i, j}\left(\alpha_{i, t}\right)$ and in turn of the SU price $s_{i, j}^{-}$in the incentive and penalty intervals. It can be seen, that as $m_{i, j}$ increases from 0 to 1 , the variance of the $\mathrm{SU}$ price curve gradually increases the $\mathrm{SU}$ price in the incentive and penalty intervals. The markers in Fig. 4.4 represent the SUs being served at the BS, and the respective prices paid by the SUs. The total bandwidth at $\mathrm{BS}_{i}$ is assumed to be $20 \mathrm{MHz}$ and each SU is allocated a fixed resource of $500 \mathrm{KHz}$ as illustrated in Fig. 4.4. 


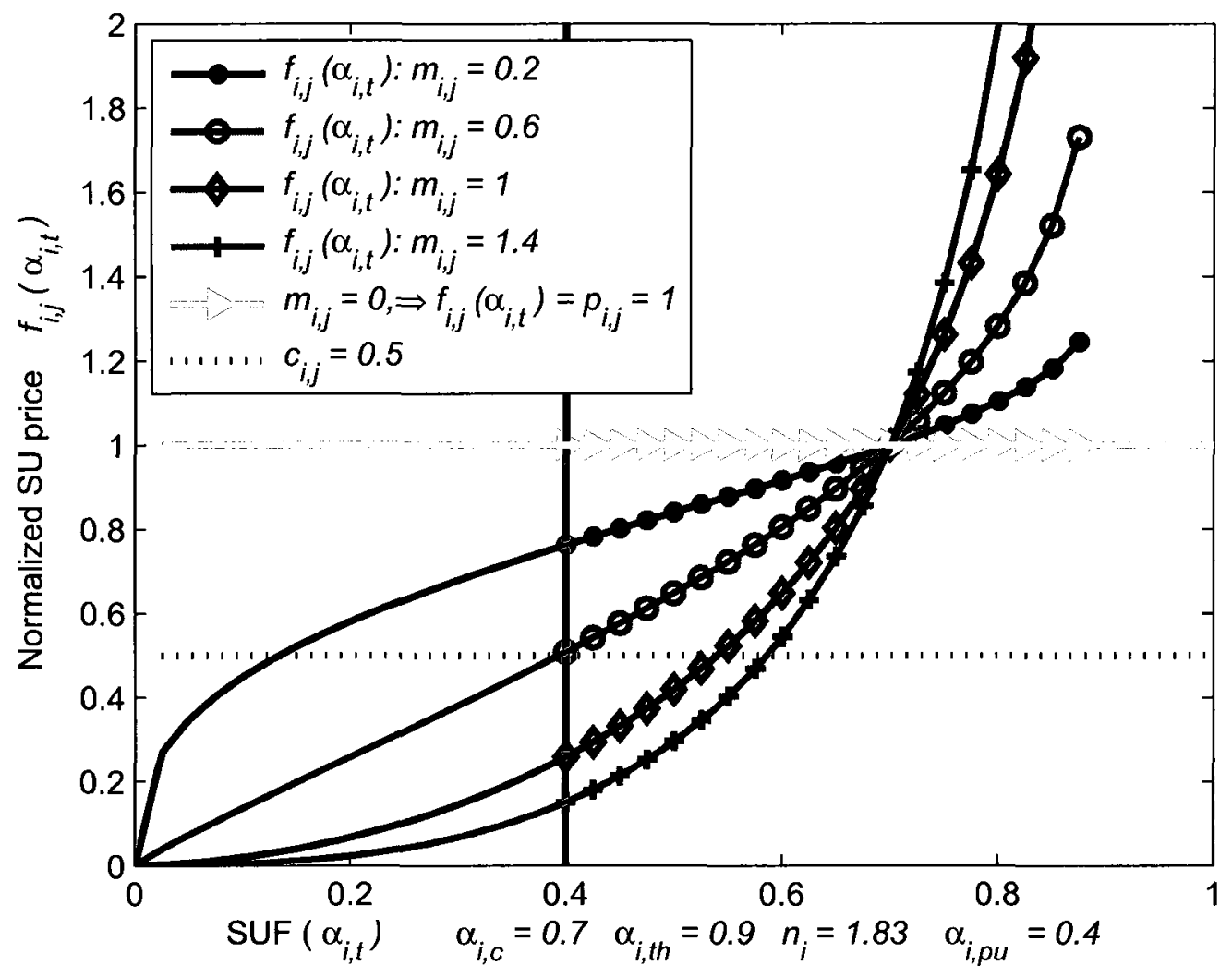

Figure 4.4: Effect of different PLFs on the normalized SU price

It can be seen from Fig. 4.3 and Fig. 4.4 that the SU price $\left(s_{i, j}=0\right)$ at $\alpha_{i, t}=0$, i.e., the SUs would stand the obtain the spectrum free of cost, when the SUF at the BS is zero (assuming WSP costs are not considered). In order to avoid this situation, a constant positive real number $s_{c}$ can be added on to (4.6) as follows:

$$
\bar{s}_{i, j}=s_{c}+\left(\left(f_{i, j}\left(\alpha_{i, t}\right)\right)^{m_{i, j}} \times\left(p_{i, j}-s_{c}\right)\right),
$$

Thus with this modification the SU price will be $s_{i, j}=s_{c}$ at $\alpha_{i, t}=0$, keeping all the configuration parameters the same. 


\subsubsection{Dynamic Nature of the Pricing Model}

The proposed pricing model based on the log barrier function has been described as dynamic throughout the thesis. This dynamic nature of SU pricing arises from the fact that the SU price varies with the SUF at $\mathrm{BS}_{i}$. However, several questions regarding the dynamic pricing model can be raised as follows:

- How often is SU price updated?

- Is this pricing model opportunistic in terms of $\mathrm{SU}$ prices?

- Does the SU price change with respect to the instantaneous values PU utilization and SUF?

This subsection is aimed at answering these questions and thus justifying the tractability and flexibility this pricing model can offer.

The SU prices will need to be updated after a certain period of time $T_{u}$, which can be considered to be in minutes. Assuming SU prices are updated, for example every 10 minutes, the update of these $\mathrm{SU}$ prices cannot be based on the instantaneous values of PU demand and SUF, as this may lead to a large difference in the SU prices every $T_{u}=10$ minutes due to the possible use of high data-rate applications at the time instance of the update. Therefore, it is reasonable to consider the average value of the PU demand and the SUF over the interval $T_{u}$, which will lead to tractable increase or decrease in the SU price. The value to be set for $T_{u}$ will be a WSP parameter, and will be needed to be set for each BS belonging to the WSP.

It must be noted that, the PU service cannot be deteriorated for allowing SU access and hence the SUs cannot be guaranteed QoS at all times as given in the RRM model from Chapter 2. 
As to the question of opportunistic nature of this pricing model, the first SU entering the BS would be charged the lowest price and the SU price will subsequently increase for every new SU entering into the system, as shown in Fig. 4.4 above. This makes the proposed pricing model opportunistic in the sense that, even tough all the SUs are provided with the same type of service, the prices charged to the SUs increase on a first-sum-first serve basis.

\subsection{Summary}

The incentive based pricing model presented in this chapter justifies the RRM model from Chapter 2, such that the SUs are provided with price incentives but may not be guaranteed QoS at all times. The basic property of the log barrier function shown in (4.3) providing inherent $\mathrm{SU}$ admission control makes the log barrier function [31] the perfect candidate for use in the incentive based pricing model. As shown in this chapter, the log barrier function can be modified to incorporate several parameters useful in modeling the pricing structure as required in the proposed distributed framework.

The key features of the incentive based pricing model can be summarized as below:

- Separate pricing model for SUs, where the SU price is represented with respect to the fixed reference PU price.

- Price incentives for SUs during periods of low PU demand at the BS.

- Inherent SU admission control, due to the use of the log barrier function in the pricing model. 
- SU price depends on spectrum utilization at the BS and PU demand.

- Configuration parameters available to the WSPs to modify the structure of the pricing model.

- Price leveling factor useful in providing flexibility in price adjustment for the WSPs.

- The pricing model is intended at developing strategic effects on SU behavior, where the SUs can anticipate and gain temporary wireless access during periods of low PU demand at the BS.

The key economic aspect aimed at proving the profitability potential of the proposed framework is presented in the next chapter. 


\section{Chapter 5}

\section{Game Theoretic Equilibrium Analysis for SU Pricing}

Assuming the technological feasibility, the successful implementation of any DSA system framework will rely on the potential for profitability to the WSPs. In the previous chapter, a dynamic incentive based pricing model for charging SUs based on PU price, PU demand and SUF at the BS was described. This chapter extends the economic perspective towards implementation of the proposed system framework and the pricing model with multiple competing WSPs in the SU wireless service market.

This chapter is organized as follows: Section 5.1 explains the rationale for the use of game theoretic equilibrium analysis for achieving pricing uniformity with competing WSPs in the SU wireless service market. Section 5.2 presents a basic literature review highlighting the previous works relevant to this chapter, and Section 5.3 describes the concept of differentiated pricing and its application in the current framework. Section 5.4 presents the linear city model for equilibrium analysis with static pricing, and Section 5.5 provides the implementation of the equilibrium analysis in achieving competitive SU pricing for the dynamic pricing model proposed in Chapter 4. Simulations and results are presented in Section 5.6 and Section 5.7 summarizes the key points from this chapter. 


\subsection{Rationale}

\subsubsection{Static Pricing v/s Dynamic Pricing}

In micro-economics, the demand curve depicts the price of a product with respect to the quantity of that product demanded or manufactured in the market, and can be used for setting the price for a particular product based on demand [32]. Therefore, the price for bread is lower than the price of gold, since the demand and availability for bread in the market is much higher than that of gold (refer Appendix A). The average price of a product generally does remain constant for a long period of time (months or years), assuming the external conditions controlling the demand and supply also remain fairly non-varying. Also the price charged to all the users or customers for that product is the same for any given point in time. Thus this type of pricing can be called as static in nature.

On the other hand, if the proposed pricing model is considered, the SU price varies with respect to $\mathrm{PU}$ demand and SUF at the $\mathrm{BS}$ and is dependent on the time and location of the BS. Thus the price for SUs at certain peak hours may be two or three times the price at off-peak hours in the same day. Also as described in Chapter 4, the pricing model is opportunistic, with the first SU gaining access to the BS being charged the lowest SU price and a higher price to be paid by each subsequent SUs. Thus this type of pricing can be considered as dynamic and opportunistic, as the price changes frequently (minutes) and the price charged to all the SUs is not the same, but gradually increases based on the order of SU entry and the SUF at the BS.

Thus this type of pricing model which is dynamic in time and order of SUs entering the system, presents a very complex problem for price setting among competing WSPs. These WSPs aiming to maximize their individual profits while simultaneously 
competing against each other in the SU wireless service market, require not only a competitive pricing structure but also need to provide incentives to attract SUs to gain temporary wireless access at their BSs in periods of low PU demand.

\subsubsection{Oligopoly and the Need for Equilibrium Analysis}

The market structure which involves a few firms in the markets competing to sell a particular product or service is known as oligopoly [32-34]. This chapter is aimed at analyzing the pricing competition among two WSPs, i.e., a WSP duopoly, for the SU wireless service market with dynamic pricing models and symmetric or asymmetric costs. For clarity in analysis throughout this chapter, the WSPs are assumed to have a single BS offering SU access. Also since each WSP operates a single BS, the index used for the BS and the WSP is the same and denoted by $i$ throughout this chapter. Symmetric costs indicate that the costs borne by both $\mathrm{WSP}_{1}$ and $\mathrm{WSP}_{2}$ are the same, while asymmetric costs indicate that WSP costs are different for the two WSPs.

Figure 5.1 depicts the scenario for WSP duopoly with symmetric costs, where the individual SU pricing models based on (4.6) with $m_{1, j}, m_{2, j}=1$ consider different configuration parameters set by the two WSPs at their BSs. The costs to the WSPs

for their BS operation are also shows in Fig. 5.1 and are assumed to be symmetric costs for both the WSPs, considering that the two WSPs are operate in the same geographical region.

\section{WSP Costs}

The costs borne by the WSPs at a particular $\mathrm{BS}_{i}$ and application class $j$ can be modelled as the sum of the CAPital EXpenditure (CAPEX) denoted by $F_{i, j}$ and OPerational EXpenditure (OPEX) denoted by $V_{i, j}\left(\alpha_{i, t}\right)$. The CAPEX includes in- 
frastructure and spectrum licensing costs which are fixed, while the OPEX include operation, maintenance and upgrading costs which are assumed to be variable with respect to spectrum utilization at the BS. The cost to a particular WSP for operation at $\mathrm{BS}_{i}$ for application class $j$ is assumed to be

$$
c_{i, j}=F_{i, j}+V_{i, j}\left(\alpha_{i, t}\right)
$$

where $F_{i, j}$ and $V_{i, j}\left(\alpha_{i, t}\right)$ are positive real numbers. It must be noted that the WSP cost is considered per SU and has the same units as the SU price and the PU price for fair comparison. The only constraint on the $\operatorname{costs} c_{i, j}$ is that the maximum cost at any SUF must be less than PU price, i.e., $\left.c_{i, j}\left(\alpha_{i, t}\right)<p_{i, j}\right)$

\section{WSP Duopoly Scenario}

The configuration parameters and the cost parameters at the $\mathrm{BS}$ for $\mathrm{WSP}_{1}$ and $\mathrm{WSP}_{2}$ are listed in Table 5.1 and Fig. 5.1 depicts the WSP duopoly scenario. It must be noted that the WSPs are assumed to have a single BS providing SU access, for purpose of analysis throughout this chapter.

Table 5.1: WSP duopoly: configuration parameters and WSP costs

\begin{tabular}{|c|c|c|}
\hline \hline Parameter & WSP $_{1}$ & WSP $_{2}$ \\
\hline Spectrum usage threshold $\alpha_{i, t h}$ & 0.9 & 0.85 \\
\hline Incentive cutoff limit $\alpha_{i, i c}$ & 0.7 & 0.75 \\
\hline Incentive cutoff factor (ICF) $n_{i, j}$ & 1.83 & 3.67 \\
\hline WSP cost $c_{i, j}=F_{i, j}+V_{i, j}\left(\alpha_{i, t}\right)$ & $0.3+0.2\left(\alpha_{i, t}\right)$ & $0.3+0.2\left(\alpha_{i, t}\right)$ \\
\hline Price leveling factor $m_{i, j}$ & 1 & 1 \\
\hline \hline
\end{tabular}

Observing the pricing curves of the two WSPs, the prices set by the WSPs for the SUs show a lot of difference due to the choice of the configuration parameters set at the BS by the WSPs, and do not present a competitive pricing structure. Also 


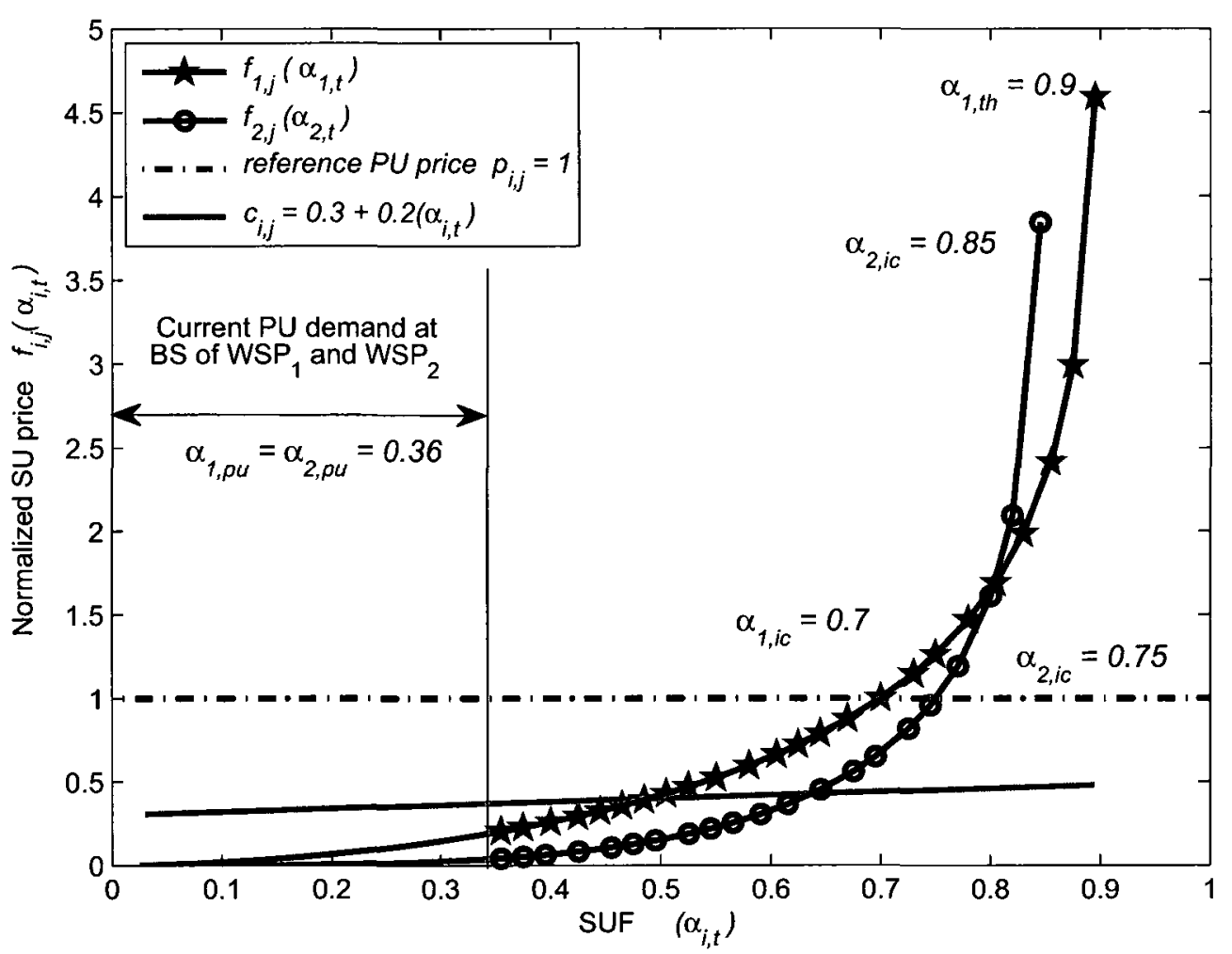

Figure 5.1: Pricing parameters: $\mathrm{WSP}_{1}$ and $\mathrm{WSP}_{2}$

SU price for some of the SUs is below the cost curve, and hence incurring a potential loss to the WSPs from those SUs. The PLF described in Chapter 4 does allows the WSPs to avoid losses, but in order to achieve competitive pricing, the use of the PLF in conjunction with the game theoretic techniques is essential.

Game theory has been the cornerstone for studying and analyzing strategic effects in the behavior of competing firms. The Nash Equilibrium (NE) provides fair prices and profits to competing firms, where there is no incentive for the individual firms to deviate from the equilibrium prices considering the pricing structure and parameters set by the other competing firms. Thus this NE SU price set by each firm is its best response with respect to the prices set by other competing firms $[33,34]$. 


\subsubsection{Cooperation v/s Non-Cooperation}

Game theory is useful in analyzing strategic situations involving individuals in groups, and in the context of this thesis WSPs in the SU wireless service market. This brings upon the key issue of cooperation among the WSPs. Although cooperation among WSPs is very unlikely due to highly competitive wireless service market, several works in literature have discussed the possibility of some type of cooperation either in relation to spectrum pooling $[14,17,19,20]$ or in terms of spectrum information sharing $[11,18]$.

Cooperation among WSPs for setting up centralized architectures to enable spectrum pooling and spectrum information pooling may also lead to the cooperation in setting the prices for SU wireless service. This type of collusion among the WSPs may lead to a cartel like structure, where the WSPs would agree on matters related to SU prices, allocation of SUs [19], allocation of spectrum bands [17,20], establishment

of common sales agencies $[17,19,20]$ and division of profits. This type of cartel or collusion for SU wireless service market in a WSP oligopoly would require cooperation among the WSPs and therefore may involve fixing prices or increasing the prices to earn higher individual profits [35].

However, this type of cooperation among WSPs is aimed at restricting competition, and may not be necessarily in the best interest of the consumers who are the SUs requiring temporary access. Therefore in this work, a distributed framework has been primarily considered, which allows individual WSPs to directly sell their spectrum to the SUs without the need for coordination and cooperation among other WSPs in the area. The same approach is followed in finding the NE SU price for the $\mathrm{SU}$ wireless market. The game theoretic analysis presented in this chapter is based on non-cooperation among the WSPs, where the WSPs are requires to set their SU 
prices simultaneously. Thus an individual WSP cannot observe the SU price of other WSPs for the next period $T_{u}$ and need to sets their own SU price, considering their best response to the predicted SU price of the other WSPs in the area.

\subsubsection{Difficulties in Equilibrium Analysis with Dynamic Pricing}

As described previously and in Chapter 4, the proposed pricing model is dynamic as different SUs are charged a different prices in the same time update period $T_{u}$, and the SU price also changes in every $T_{u}$ based on the SUF at the BS.

Game theoretic analysis for NE based pricing is easier with static pricing, since the price once fixed is same for all users and customers, and the NE price once found remains fairly constant for long time periods (months, years). With the price charged to all the users or customers being the same, the equilibrium analysis is tractable and it is possible to obtain the best response to the prices set by other firms.

However, in case of dynamic pricing as in the proposed pricing model, since the price charged for all the SUs is different, it is very difficult and may be prohibitively complex to find the best responses to the changing prices. As seen in Fig. 4.4 from Chapter 4, the PLF allows the WSPs to vary and adjust the SU prices using (4.6), and the SU price depends on the PU demand and the SUF at the BS.

With the WSPs having different configuration parameters as shown in Fig. 5.1 and possibly different WSP costs and different PU demands (considered in the simulations and results in Section 5.5), finding the SU price at a particular SUF at one BS may or may not be the best response to the SU price at the same SUF at the BS of the other WSP.

Thus due to the above predicament arising due to the use of dynamic pricing, the following strategy is followed in order to achieve competitive pricing among WSPs. 


\subsubsection{Strategy for Achieving Inter-WSP Competitive Pricing}

Using the PLF from (4.6), each individual WSP can adjust their pricing model with respect to the PU demand at their BSs, keeping the configuration parameters unchanged. Therefore a unique method for the implementation of the dynamic pricing model using the PLF based on static NE SU price is developed in this chapter.

Although no claims for equilibrium can be made, the results based on this strategy presented in Section 5.6 show competitive pricing among WSPs, with guaranteed individual WSP profits and without assuming any type of cooperation among the WSPs.

The procedure followed in achieving such type of competitive pricing among WSPs for SU wireless service is listed below and will be elaborated in the following sections:

- SU service differentiation based on the wireless channel (Section 5.3 and Section 5.4.1)

- Equilibrium analysis with static pricing based on SU service differentiation (Section 5.4.2)

- Implementation of static NE SU price obtained from the above equilibrium analysis onto the proposed dynamic pricing model using the PLF (Section 5.4.3)

This procedure to achieve competitive pricing among WSPs can be considered as one of the key contributions of this chapter. The other key contribution is the demonstration of the potential for profit to the WSPs from SU access based on the proposed framework as will be shown in Section 5.6. Before moving on the process 
for achieving competitive pricing among WSPs for SU wireless service, the literature review related to this chapter is presented.

\subsection{Literature Review and Rationale}

SU pricing mechanisms and their equilibrium analysis considering a CR network has been considered in [11]. Three types of market mechanisms were compared in [11], with varying levels of cooperation among the WSPs. The SU service is also assumed to cause possible deterioration to PU service, and the SUs are also charged an extra fee for compensation in [11]. A overview of the various market models, methods for equilibrium analysis from the perspective of SU wireless access market have also been presented in [36].

This work is significantly different from that proposed in [11], since the joint PU-SU RRM model as described in Chapter 2 ensures no deterioration to PU service due to SU access. Also the most general case of no-cooperation among the WSPs in setting the $\mathrm{SU}$ prices is assumed in this thesis as discussed previously. The dynamic pricing model presented in this thesis also provides incentives to the SUs in periods of low PU demand. Although no claims can be made for theoretical equilibrium, competitive pricing among WSPs is achieved empirically by the implementation of static equilibrium analysis on the dynamic pricing model using the PLF, and is one of the novel contributions from this chapter.

\subsection{Oligopoly and SU Wireless Service Differentiation}

Assuming two WSPs in a region provide identical wireless service to the PUs

and the WSPs compete on prices, the NE price per subscription can be shown to be equal to the marginal cost of providing the wireless service, thus making zero 
individual WSP profits [33] (refer Appendix A). However, in reality, the WSPs do earn profits by differentiating their wireless service subscription from other WSPs in the region. This differentiation can be based on several service features such as the add-on services provided with the subscription, the general level of network coverage of a WSP, brand name and other factors, which influence the WSP selection decision of the potential subscribers (potential PUs), apart from the basic subscription price.

Considering the SU market, since the SUs are provided temporary wireless access, the criterion for network (BS) selection is based on two basic features namely the SU price $s_{i, j}$ and the average achievable link spectral efficiency $\eta_{i}$ estimated based on the SNR observed at the SU terminal. The procedure and criterion for network (BS) selection has been described in Chapter 3. As the system framework has a distributed nature, it must be stressed that the SU terminal actually selects the most appropriate BS and in turn the WSP during the network selection process.

Thus apart from the price charged by the WSPs for temporary access, the critical parameter considered by the SUs before WSP selection is $\eta_{i}$. Assuming the prices set by the WSPs to be close, the key differentiating factor for the SUs during network selection is primarily $\eta_{i}$ and is based on the location and the distance of the SU terminal from the $\mathrm{BS}$ of $\mathrm{WSP}_{i}$.

This differentiation of the SU wireless service based on location of the SU terminal, i.e, the wireless channel, inherently distinguishes the SU wireless service offered by the BSs of the WSPs in the area. The wireless channel thus allows the WSPs to avoid the scenario of zero profits, where the price set by the WSPs considers identical service without any service differentiation.

The rationale behind SU wireless service differentiation is thus based on the variability of the wireless channel and assures profits to the WSPs from SU access. 
This idea of differentiated SU wireless service is used in the next section for game theoretic equilibrium pricing analysis with static pricing in a WSP duopoly using the linear city model. It must be noted that the following section provides the intermediate step for incorporating competitive pricing into the SU dynamic pricing model.

\subsection{Game Theoretic Analysis for SU Pricing}

\subsubsection{Linear City Model}

In order to perform equilibrium analysis for two WSPs with differentiated SU service, a simple scenario of a linear city is considered for ease of analysis. The linear city model for differentiated SU wireless service considers a city to be a line of distance 1 unit. It must be noted that, throughout this chapter, the index $i$ is used to denote the WSP since each WSP is assumed to have a single BS. The single BS of each WSP is placed at either side of this city at a distance $d f_{i}$ from the city, where $d f_{i}$ is the exclusion region and depends on antenna configurations at the BS of $\mathrm{WSP}_{i}$. The unit distance of the linear city is represented by $y \in[0,1]$ and starts from the exclusion region $d f_{i}$ of the $\mathrm{BS}$ of $\mathrm{WSP}_{i}$ to the cell edge. Thus $y=0$ at $d f_{1}$ and $y=1$ at cell edge for $\mathrm{WSP}_{1}$ and vice versa for $\mathrm{WSP}_{2}$ as shown in Fig. 5.2.

In order to derive the SU demand for wireless service at the two WSPs, the SUs are considered to be uniformly distributed along the city, with a single SU placed at every point $q$ along the line from 0 to 1 . Each of the $q$ SUs are required to select either of the two WSPs for obtaining wireless access for a finite block of $B$ bytes, thus maintaining the demand exactly equal to 1 .

Since the aim of this section is to find the NE SU price $S_{i, j}^{*}$ considering a static 


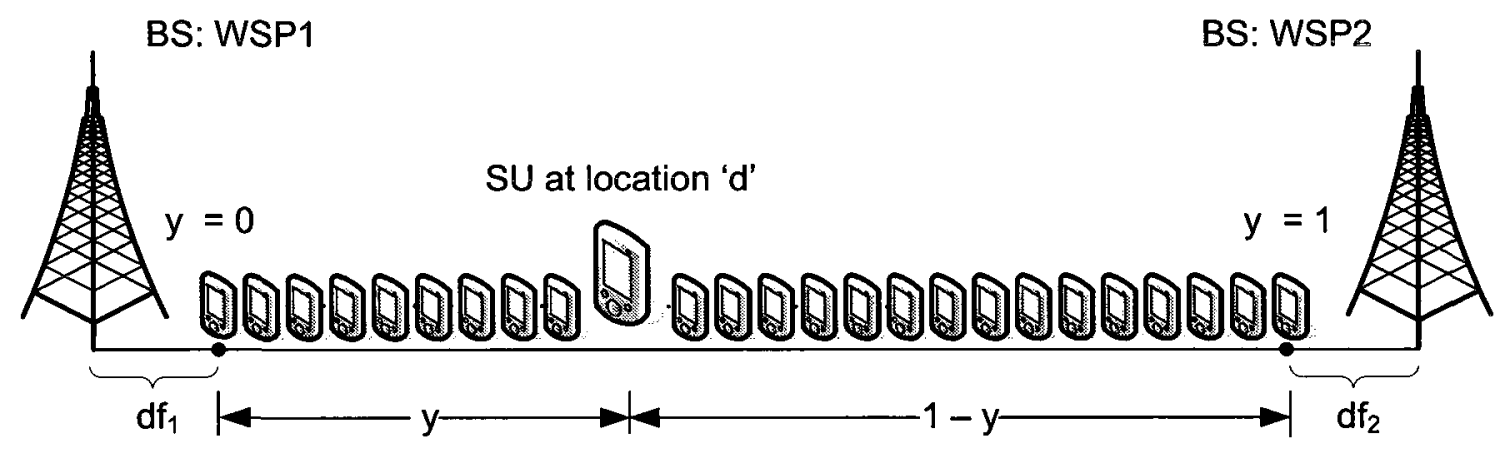

Figure 5.2: Linear city model

pricing model, the SU price $S_{i, j}$ for the first $\mathrm{SU}$ joining the $\mathrm{BS}$ of $\mathrm{WSP}_{i}$, i.e., at $\alpha_{i, p u}=\alpha_{i, t}$, is considered as a fixed SU price for all the SUs in the linear city. This static SU price for the SUs currently located in the linear city is also assumed to be the discounted price, i.e., $S_{i, j}<p_{i, j}$. Therefore from the spectrum utilization standpoint, the SUF at the $\mathrm{BS}$ of $\mathrm{WSP}_{i}$ is considered to be in the incentive region, i.e., $\alpha_{i, t} \in\left(\alpha_{i, p u}, \alpha_{i, i c}\right)$, based on the configuration parameters set by the WSPs. Furthermore, from the SU demand standpoint, the BSs of both the WSPs are assumed to have sufficient total residual spectrum in $\left(\alpha_{i, p u}, \alpha_{i, i c}\right)$ to together provide temporary wireless access to all the $q$ SUs in the city.

Similar to static SU price, the WSP cost per SU at the BSs of the WSPs is considered to be fixed and equal for both the WSPs and is given as $C_{j}=C_{1, j}=C_{2, j}$. These assumptions of static SU pricing and fixed WSP cost are relaxed when NE SU price that will be obtained from the following equilibrium analysis is applied to the incentive based pricing model for achieving competitive dynamic pricing.

The static SU prices $S_{1, j}$ and $S_{2, j}$ are assumed to be simultaneously set by both the WSPs, thus one WSP cannot observe the other WSPs price before setting his own SU price. Thus the NE SU price needs to be less than PU price thus providing discounted pricing to the $\mathrm{SU}$, while greater than the WSP cost per SU thus generating 
profits for the WSPs from SU access, i.e., $C_{j}<S_{i, j}<p_{i, j}$.

Before starting with the equilibrium analysis, the fundamental assumption in economics and game theory needs to be noted that both the WSPs are rational and aim to maximize their individual profits [32-34].

\subsubsection{Equilibrium Analysis for SU Pricing}

For the SU equilibrium pricing analysis with differentiated SU wireless service, the perceived price for temporary wireless access from $\mathrm{BS}$ of $\mathrm{WSP}_{i}$ to the $\mathrm{SU}$ located at any distance $y$ on the linear city for application class $j$ can be given as

$$
U_{i, j}(y)=S_{i, j}+(\zeta \times y) \quad(\$)
$$

where $\zeta$ is a constant nonnegative real number representing the dissatisfaction price $(\$)$ to a $\mathrm{SU}$, such that the perceived price to the SU increases as distance $y$ of the SU from BS of $\mathrm{WSP}_{i}$ increases. This dissatisfaction price is based on the wireless channel and is described in the next section. Considering the SU located at a distance $y$ from $\mathrm{BS}$ of $\mathrm{WSP}_{1}$ and at a distance of $1-y$ from $\mathrm{BS}$ of $\mathrm{WSP}_{2}$ as shown in Fig. 5.2, the $\mathrm{SU}$ will select $\mathrm{WSP}_{1}$ for temporary wireless access provided

$$
U_{1, j}(y)<U_{2, j}(1-y)
$$

while will select $\mathrm{WSP}_{2}$ if

$$
U_{1, j}(y)>U_{2, j}(1-y)
$$

For the $\mathrm{SU}$ to which the two perceived $\mathrm{SU}$ prices are found to be equal, i.e., $U_{1, j}(y)=$ $U_{2, j}(1-y)$, the selection of the WSP will be based on a random selection [33].

Thus $\mathrm{WSP}_{1}$ could potentially capture the entire market by setting $y=1$ in (5.3) such that $S_{1, j}<S_{2, j}-\zeta$, i.e., serve all the $q$ SUs in the linear city, if hypothetically the 
price set by $\mathrm{WSP}_{2}$ is known by $\mathrm{WSP}_{1}$. However, in context of the system framework and the assumptions described, due to limited quantity of spectrum to offer to the SUs, the entire SU demand cannot be satisfied by a single WSP, and the SU prices are assumed to be set simultaneously. Also considering the case of the dynamic pricing model, since the SU prices are incremental there will never be a single WSP that will be able to capture the entire SU wireless service market.

Since it will not be possible for either of the WSPs to capture the entire market by out-pricing the other, the two WSPs thus need to set SU prices close to each other assuming the SUs to be divided between the two WSPs. The following analysis is aimed at finding the NE SU price such that SU prices set by both the WSPs are close and competitive, as well as individual profits to the WSPs are guaranteed.

Assuming the fixed SU prices set by the two WSPs will be close, a particular SU located at a distance $d$ on the linear city is considered, such that $U_{1, j}(d)=U_{2, j}(1-d)$. Solving the equality for $d$, the SU demand for connection to $\mathrm{WSP}_{1}$ can be given as

$$
D_{1, j}\left(S_{1, j}, S_{2, j}\right)=\frac{\zeta-S_{1, j}+S_{2, j}}{2 \zeta}
$$

where $D_{1, j}\left(S_{1, j}, S_{2, j}\right)$ is a nonnegative real number and represents the SU demand for $\mathrm{WSP}_{1}$ as a function of the $\mathrm{SU}$ prices set by the two WSPs.

Thus the profit achievable from SU access by $\mathrm{WSP}_{1}$ based on the above $\mathrm{SU}$ demand is given as

$$
\pi_{1, j}\left(S_{1, j}, S_{2, j}\right)=S_{1, j} \times\left(D_{1, j}\left(S_{1, j}, S_{2, j}\right)\right)-C_{j} \times\left(D_{1, j}\left(S_{1, j}, S_{2, j}\right)\right)
$$

where $\pi_{1, j}\left(S_{1, j}, S_{2, j}\right)$ is a nonnegative real number and represents the profit to $\mathrm{WSP}_{1}$ from $\mathrm{SU}$ access as a function of SU prices set by the two WSPs.

Profit maximization for $\mathrm{WSP}_{1}$ requires $\mathrm{WSP}_{1}$ to find the best response $\mathrm{SU}$ price to be set in response to the $\mathrm{SU}$ price set by $\mathrm{WSP}_{2}$ denoted as $B R_{1, j}\left(S_{2, j}\right)$. Finding 
the best response of $\mathrm{WSP}_{1}$ requires the differentiation of (5.6) with respect to the $\mathrm{WSP}_{1} \mathrm{SU}$ price $S_{1}$ to obtain the first order conditions [33] as follows:

$$
B R_{1, j}\left(S_{2, j}\right)=S_{1, j}=\frac{\partial \pi_{1, j}}{\partial S_{1, j}}=\frac{S_{2, j}+\zeta+C}{2} .
$$

Assuming the model to be symmetric, a similar set of results can be obtained for $\mathrm{WSP}_{2}$.

Thus the NE SU price can be expressed as follows:

$$
S_{j}^{*}=C_{j}+\zeta
$$

where $S_{j}^{*}$ is non-negative real number representing the NE SU price considering the $\mathrm{SU}$ prices set by the WSPs to be equal to each other $S_{j}^{*}=S_{1, j}^{*}=S_{2, j}^{*}$ [33]. It can be seen from (5.8), that the dissatisfaction price $\zeta$ differentiates the SU wireless service provided by each WSP, thus enhances the profits to both WSPs.

\section{SU Dissatisfaction Price}

The dissatisfaction price of the $\mathrm{SU}$ is defined as a measure of the dissatisfaction of the SU with respect to the signal strength received at the SU terminal from the $\mathrm{BS}$ of $\mathrm{WSP}_{i}$. This parameter is defined as a system parameter and is modeled in terms of the variability of the wireless channel based on the Satisfaction Level $S L_{i}$ of the SUs in the linear city.

The Satisfaction Level $S L_{i}(r)$ for the $r^{\text {th }} \mathrm{SU}$ on the linear city with respect to $\mathrm{BS}$ of $\mathrm{WSP}_{i}$ can be defined as the normalized value of the average achievable link spectral efficiency $\eta_{i}(r)$ of the $r^{\text {th }} \mathrm{SU}$ with respect to the maximum $\eta_{i}$, and is given as

$$
S L_{i}(r)=\frac{\eta_{i}(r)}{\max \left(\eta_{i}\right)}
$$


where $S L_{i}(r)$ is a non-negative real number. Thus considering shadowing to be averaged out, the maximum value of $\eta_{i}$ can be observed at $d f_{1}$, i.e., at $y=0$ nearest to BS of $\mathrm{WSP}_{i}$, and the $S L_{i}$ decreases as the $y$ increases from 0 to 1 .

The standard deviation of $S L_{i}$ denoted as $\sigma_{i}$ is used to model the variability of wireless channel considering the positions of the $q$ SUs uniformly distributed over the linear city, and can be given as

$$
\sigma_{i}=\sqrt{\frac{1}{q-1} \sum_{r=1}^{q}\left(S L_{i}(r)-S \bar{L}_{i}\right)^{2}},
$$

where $S \bar{L}_{i}$ denotes the mean satisfaction level for the SUs on the linear city.

The dissatisfaction price $\zeta$ is defined in terms of the standard deviation of the wireless channel and is given as

$$
\zeta=K_{1} K_{2} \quad(\$)
$$

where $K_{1}$ and $K_{2}$ are constants and are nonnegative real numbers.

Assuming the monetary price paid by the SU per unit change in $\sigma_{i}$ with respect to $\mathrm{WSP}_{i}$ as unity, the two constants $K_{1}$ and $K_{2}$ are given as follows:

$$
K_{1}=1 \quad(\$) ; \quad K_{2}=\left(\frac{\sigma_{1}+\sigma_{2}}{2}\right)
$$

It must be noted that the calculation of the value of $\zeta$ needs to be performed only once for a particular competitive scenario and wireless environment.

Case of Perfect Substitutes $\zeta=0$

Considering the hypothetical case of unlimited power at the BS and zero noise over the linear city, the satisfaction level $S L_{i}$ for all the SUs is constant and therefore $\sigma=0$. Thus from (5.2), $\zeta=0$ implies that all the SUs on the linear city are 
indifferent in selection of $\mathrm{WSP}_{1}$ or $\mathrm{WSP}_{2}$. The two WSPs can be thus considered as perfect substitutes, resulting in perfect inter-WSP competition bringing down the WSP profits to zero [33] (refer Appendix A).

It can thus be inferred from (5.8) that, an increase in the dissatisfaction price $\zeta$ resulting from the increase in the variability of the wireless channel in the linear city is beneficial to the WSPs. The variability of the wireless channel inherently provides SU wireless service differentiation between the two WSPs, thus increasing WSP profits earned through SU access.

\subsubsection{Implementation to the Dynamic Pricing Model}

In this section, the implementation of NE SU price obtained from the above equilibrium analysis in (5.8) onto the proposed dynamic pricing model using the PLF is developed. The assumption of static SU price and static WSP cost per SU, which was used for the equilibrium analysis considering the linear city model is relaxed. The aim in this section, is to find the value of the PLF for incorporating NE SU price from the previous section in order to achieve competitive pricing among WSPs. For clarity and convenience of the reader, equation (4.6) from Chapter 4 defining the SU price with PLF is repeated below:

$$
\bar{s}_{i, j}=\left(f_{i, j}\left(\alpha_{i, t}\right)\right)^{m_{i, j}} \times p_{i, j} .
$$

This value of the PLF is individually found at the $\mathrm{BS}$ of $\mathrm{WSP}_{i}$ by rearranging (5.13) and setting $\bar{s}_{i, j}=S_{i, j}^{*}$ and $\alpha_{i, t}=\alpha_{i, p u}$, as

$$
\overline{m_{i, j}}=\frac{\ln \left(\frac{S_{i, j}^{*}}{p_{i, j}}\right)}{\ln \left(f_{i, j}\left(\alpha_{i, p u}\right)\right)},
$$

where $S_{i, j}^{*}=C_{i, j}+\zeta$ based on the (5.8). Thus the PLF is defined based on a single

point which maps the NE SU price to the SUF where the first SU enters the system, 
i.e., $\alpha_{i, t}=\alpha_{i, p u}$. This transformation gives the value of the PLF, which is then replaced in (4.6) or in (5.13) to generate dynamic pricing considering the static NE SU price.

It must be noted that the PLF allows that NE pricing to be implemented, and setting $m_{i, j}=1$ reverts back to the basic pricing model based on the configuration parameters shown in Fig. 5.1.

Apart from the PLF, the SU price and the SU profits further depend on the configuration parameters $\alpha_{i, i c}$ and $\alpha_{i, t h}$. It can be observed that, the willingness to pay of the SUs, the wireless environment, and the SUF at the BS are together considered in the final price the SUs' pay for temporary wireless access, which also guarantees profits to the WSPs.

\subsection{Simulations and Results}

This section first describes the channel model used in the linear city and then presents the performance results for profits gained by the WSPs in four different scenarios. The simulations related to the channel model and pricing and profit analysis were performed using the Matlab software.

\subsubsection{Channel Model for the Linear City}

A simple scenario with a single BS per WSP in a single cell without inter-cell interference is used to model the wireless environment on the linear city for both the WSPs. The carrier frequencies of $2000 \mathrm{MHz}$ and $1900 \mathrm{MHz}$ for $\mathrm{WSP}_{1}$ and $\mathrm{WSP}_{2}$ respectively have been assumed. Hence the distance based path loss model can be given as $P L_{1}=128.1+37.6 \log _{10}(\mathfrak{d})$, for $\mathrm{WSP}_{1}$ while $P L_{2}=127.7+37.6 \log _{10}(\mathfrak{d})$ for

$\mathrm{WSP}_{2}$, where $\mathfrak{d}$ is the distance in meters. An urban macro-cell scenario from [37] is 
Table 5.2: Channel model: simulation parameters

\begin{tabular}{|c|c|}
\hline \hline Parameter & WSP $_{1} / \mathbf{W S P}$ \\
\hline BS-cell edge distance & $250 \mathrm{~m}$ \\
\hline Exclusion distance $d f_{i}$ & $35 \mathrm{~m}$ \\
\hline BS total transmit power & $46 \mathrm{dBm}$ \\
\hline BS transmitter antenna gain & $14 \mathrm{~dB}$ \\
\hline SU terminal receiver antenna gain & $0 \mathrm{~dB}$ \\
\hline Noise figure & $5 \mathrm{~dB}$ \\
\hline Noise power density at SU terminal & $-174 \mathrm{dBm} / \mathrm{Hz}$ \\
\hline Total bandwidth & $20 \mathrm{MHz}$ \\
\hline Target BER & $10^{-6}$ \\
\hline \hline
\end{tabular}

considered as the basis for the path loss model for the linear city, and the simulation parameters are listed in Table 5.2.

A scatter plot of the average achievable link spectral efficiencies for $\mathrm{WSP}_{1}$ and $\mathrm{WSP}_{2}$ from $\mathfrak{d}=35 \mathrm{~m}$ up to $\mathfrak{d}=250 \mathrm{~m}$ is shown in Fig. 5.3 . In order to average over the shadowing experienced by the SUs in the linear city, the scatter plot in Fig. 5.3 has been generated from a 1000 drops, and is approximated by a $3^{\text {rd }}$ degree polynomial using least-square curve fitting.

\subsubsection{Performance Results}

The objective of this chapter has been to demonstrate the potential for additional profits to the WSPs from SU access based on the proposed framework and pricing model. The prices charged by the WSPs to the SUs and the potential additional profits that can be earned by the WSPs in four different scenarios is presented in this subsection.

The performance analysis consider a model similar to the linear city with two $\mathrm{WSP}_{\mathrm{s}}$, i.e., $\mathrm{WSP}_{1}$ and $\mathrm{WSP}_{2}$, having a single BS and competing with each other for the SU wireless service market. The following simulations and results only consider 


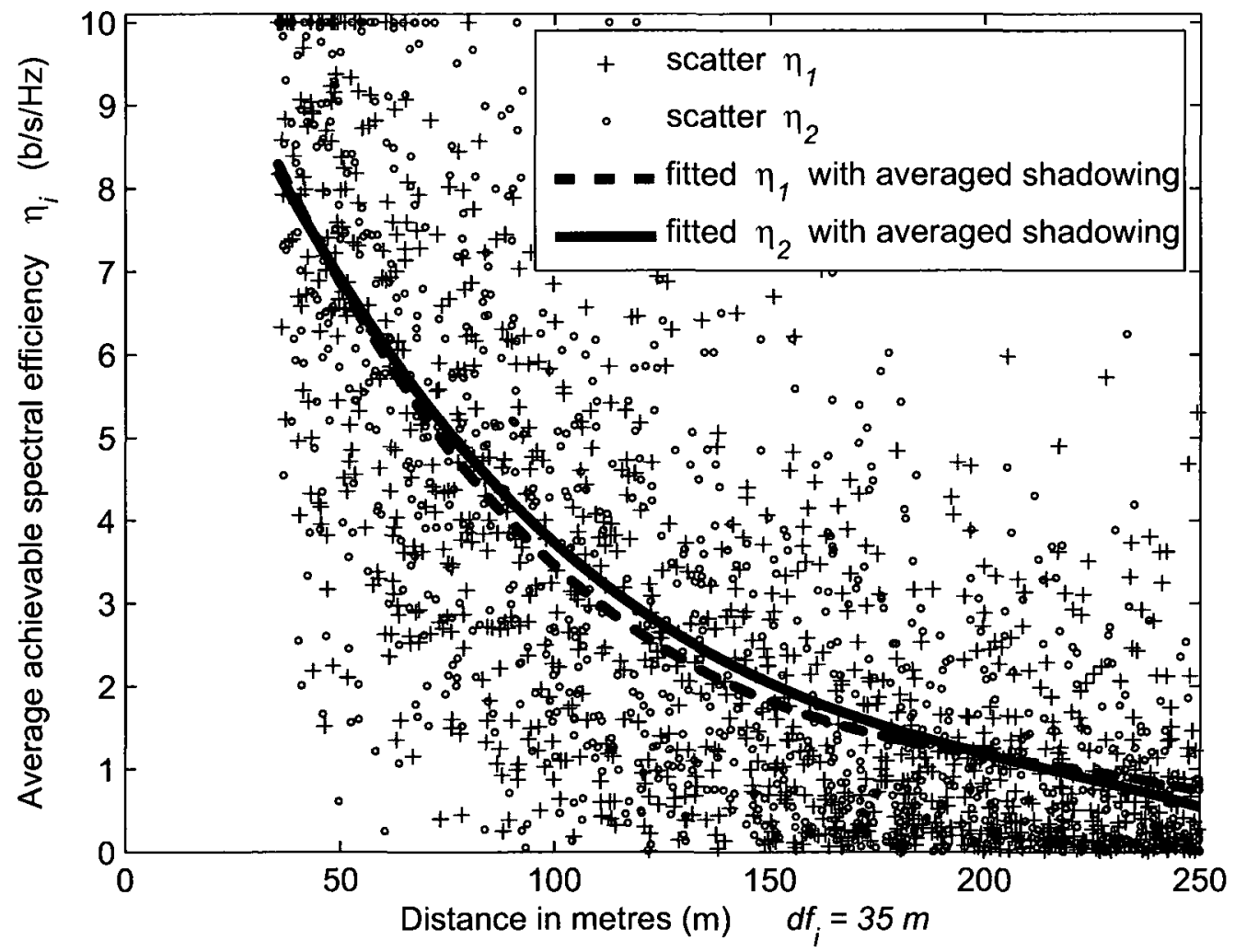

Figure 5.3: Spectral efficiencies over the linear city

the SUs with monetary incentive $\left(s_{i, j}<p_{i, j}\right)$, i.e., the SUs gaining access in the incentive region $\left(\alpha_{i, p u}, \alpha_{i, i c}\right)$. Also each of these SUs are randomly allocated either 400 or 500 or $600 \mathrm{KHz}$, assuming the bandwidth at the BSs of both the WSPs to be $20 \mathrm{MHz}$. Therefore in these simulations, the number of SUs allowed into the network for the time period of $T_{u}$ between the SU price updates depends upon the following:

- The randomly allocated bandwidth to each SU (e.g. 400, 500 or $600 \mathrm{KHz}$ )

- The fixed bandwidth at the BS (e.g. $20 \mathrm{MHz}$ )

- The average PU demand $\left(\alpha_{i, p u}\right)$ 
- The incentive cutoff limit $\left(\alpha_{i, i c}\right)$

The WSPs need to consider the average PU demand at the BS and the WSP cost at the BS before setting the value of PLF. As described previously, the PLF value set by each WSP is based on (5.8), i.e., considering the static NE SU price obtained from equilibrium analysis.

The following four scenarios based on the PU demand and the WSP cost are used for analyzing the profit potential to the WSPs from SU access:

Scenario 1: Similar average PU demand and symmetric WSP costs

Scenario 2: Similar average PU demand and asymmetric WSP costs

Scenario 3: Dissimilar average PU demand and symmetric WSP costs

Scenario 4: Dissimilar average PU demand and asymmetric WSP costs

Two plots are presented for each of the above scenario, with the first plot illustrating the SU prices set by the individual WSPs at their BSs in the incentive region, and the second plot depicting the Cumulative Profit (CP) that can be obtained by the WSPs at one BS. In the first plot, the SU price curves are initially generated based on (5.13), (5.14) and (5.8), after which the markers denoting the SUs with randomly allocated bandwidths of either $400 \mathrm{KHz}$ or $500 \mathrm{KHz}$ or $600 \mathrm{KHz}$ are superimposed on the same plot. It must be noted that the bandwidth at the BS of the WSPs is assumed to be $20 \mathrm{MHz}$, as stated previously. The basic configuration parameters for the two WSPs are assumed to be the same as in Table 5.1 and Fig. 5.1. Both the plots analyze the SU prices set by the two WSPs for the three cases listed as follows:

Case 1: The basic pricing model with PLF $=1$ based on (4.6) and $\zeta=0$. 
Case 2: The pricing model where PLF is calculated using (5.14) and (5.8), but without SU wireless service differentiation, i.e., for $\zeta=0$ (perfect substitutes).

Case 3: The pricing model where PLF is calculated using (5.14) and (5.8), and the value of $\zeta$ is calculated using (5.11).

Assuming a total of $l$ SUs being allowed access to the BS of $\mathrm{WSP}_{i}$ in the incentive region, the $\mathrm{CP}$ for $\mathrm{WSP}_{i}$ is a sequence of partial profits corresponding to the sequence

$$
\left\{\overline{s_{i, j}}(1), \overline{s_{i, j}}(2), \overline{s_{i, j}}(3), \ldots, \overline{s_{i, j}}(l)\right\}
$$

and can be given as $\left\{\overline{s_{i, j}}(1),\left(\bar{s}_{i, j}(1)+\bar{s}_{\bar{i}, j}(2)\right),\left(s_{i, j}^{-}(1)+\overline{s_{i, j}}(2)+\overline{s_{i, j}}(3)\right), \ldots,\left(\overline{s_{i, j}}(1)+\right.\right.$ $\left.\left.\bar{s}_{i, j}(2)+\overline{s_{i, j}}(3)+\ldots+\bar{s}_{i, j}(l)\right)\right\}$.

The following SU pricing and WSP CP plots for the above scenarios will show that, the SU prices and the WSP CPs for case 1 are the lowest, followed by case 2 and finally case 3 . Case 3 incorporates NE SU pricing with SU service differentiation based on the wireless channel. The competitive trend in the SU pricing and the WSP CPs can be observed through the closeness in the case 3 curves for the two plots and will be evident from the plots for the above scenarios. It must be noted that, due to asymmetric WSP costs and dissimilar PU demands in certain scenarios, the SU pricing curves may not appear to be close, but the actual SU prices in the respective scenarios will be observed to be close thus justifying the competitive trend.

Based on the cases considered and the description in this chapter, the SU price and the WSP profits from SU access will be observed to increase in ascending order from case 1 to case 3 . The plots for the four scenarios along with a table summarizing the results in each scenario are presented next. For organization and clarity, each scenario is presented on separate pages starting from the next page. 
Scenario 1: Similar Average PU Demand and Symmetric WSP Costs

In this scenario, the PU demand at each of the BSs of the two WSPs is assumed to be 0.355 and 0.39 respectively as shown in Table 5.3 . The costs borne by both the WSPs are assumed to be symmetric and equal to $c_{i, j}=0.3+0.2\left(\alpha_{i, t}\right)$. It can be seen from case 3 of Fig. 5.4 that the adjusted SU prices are fairly similar and are dictated by the configuration parameters set at each of the BS. The CP to both the WSPs are also seen to be very close to each other in case 3 of Fig. 5.5 and hence are observed to be competitive.

Table 5.3: Scenario 1: WSP parameters and results

\begin{tabular}{|c|c|c|}
\hline \hline Parameter & WSP $_{1}$ & $\mathbf{W S P}_{2}$ \\
\hline PU demand $\alpha_{i, p u}$ & 0.355 & 0.39 \\
\hline Spectrum threshold $\alpha_{i, t h}$ & 0.9 & 0.85 \\
\hline Incentive cutoff limit $\alpha_{i, i c}$ & 0.7 & 0.75 \\
\hline Incentive cutoff factor $n_{i, j}$ & 1.83 & 3.66 \\
\hline WSP cost & $0.3+0.2\left(\alpha_{1, t}\right)$ & $0.3+0.2\left(\alpha_{2, t}\right)$ \\
\hline \hline Results & $\mathbf{W S P}_{1}$ & $\mathbf{W S P}_{2}$ \\
\hline NE SU price with $\zeta=0.25(\$)$ & 0.63 & 0.63 \\
\hline SUs served & 14 & 15 \\
\hline CP with $\zeta=0.25(\$)$ & 5.57 & 5.94 \\
\hline \% rise in CP for $\zeta=0.25$ w.r.t. case 2 & 41.61 & 39.58 \\
\hline \% rise in CP for $\zeta=0.25$ w.r.t. case 1 & 72.53 & 112.99 \\
\hline \hline
\end{tabular}




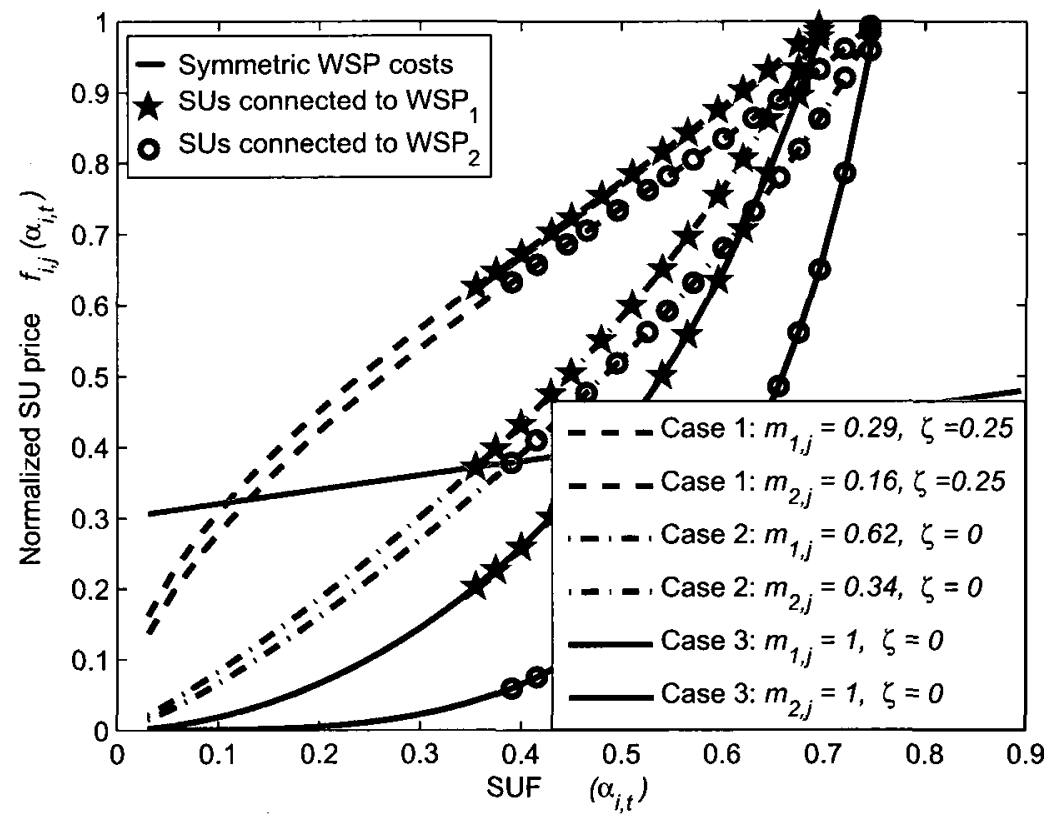

Figure 5.4: Scenario 1: SU price set by the two WSPs

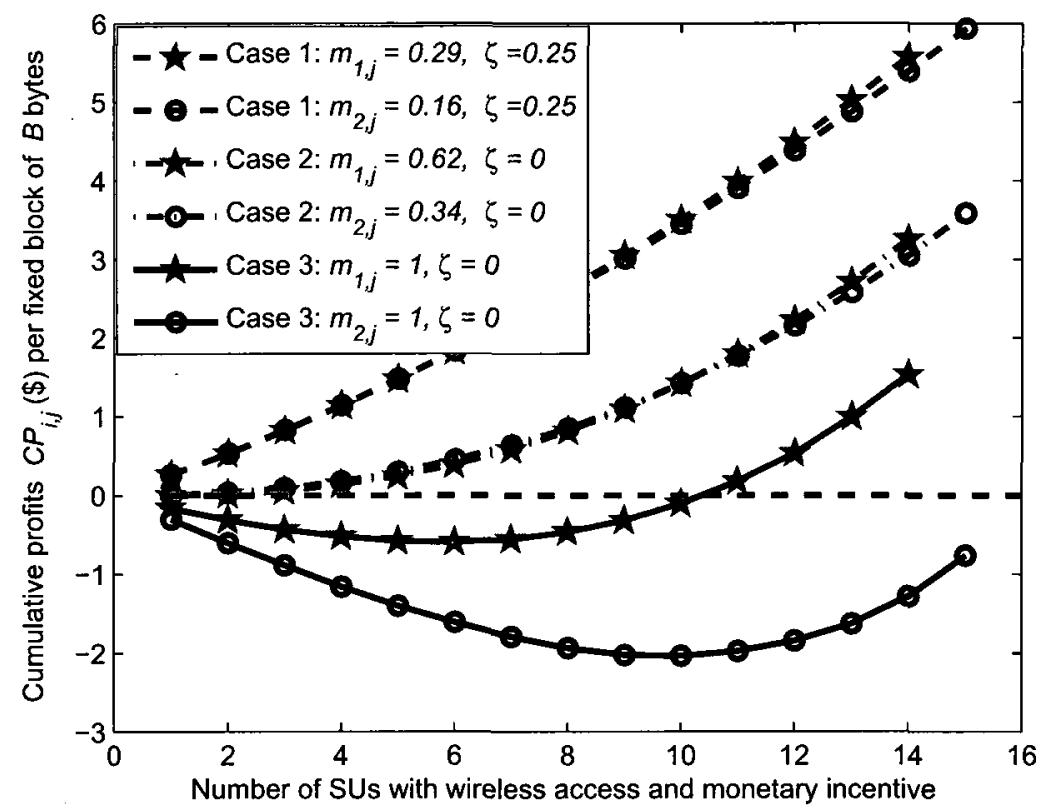

Figure 5.5: Scenario 1: CP obtained by the two WSPs from SU access 
Scenario 2: Similar Average PU Demand and Asymmetric WSP Costs

In this scenario, the PU demand at each of the BSs of the two WSPs are again assumed to be fairly similar, i.e., 0.305 and 0.33 respectively as shown in Table 5.4. The costs borne by both the WSPs are assumed to be asymmetric therefore the $\mathrm{WSP}_{1}$ costs are given as $c_{1, j}=0.3+0.2\left(\alpha_{1, t}\right)$, while $\mathrm{WSP}_{2}$ costs are defined by $c_{2, j}=0.1+0.7\left(\alpha_{2, t}\right)$. It can be seen from the cost equations and Fig. 5.6, the slope of the two costs does vary widely. However, in spite of the widely varying costs, the adjusted SU prices in case 3 of Fig. 5.6 can be seen to be fairly similar. The CP for $\mathrm{WSP}_{1}$ is lower than the CP for $\mathrm{WSP}_{2}$, as the slope of $\mathrm{WSP}_{1}$ cost is higher and also the average $\mathrm{WSP}_{1}$ cost to the $\mathrm{SUs}$ is higher than for $\mathrm{WSP}_{2}$. Therefore $\mathrm{WSP}_{2}$ does enjoy slightly higher profits, but the SU pricing and WSP profits can be still seen to be highly competitive.

Table 5.4: Scenario 2: WSP parameters and results

\begin{tabular}{|c|c|c|}
\hline \hline Parameter & $\mathbf{W S P}_{1}$ & $\mathbf{W S P}_{2}$ \\
\hline PU demand $\alpha_{i, p u}$ & 0.305 & 0.33 \\
\hline Spectrum threshold $\alpha_{i, t h}$ & 0.9 & 0.85 \\
\hline Incentive cutoff limit $\alpha_{i, i c}$ & 0.7 & 0.75 \\
\hline Incentive cutoff factor $n_{i, j}$ & 1.83 & 3.66 \\
\hline WSP cost & $0.3+0.2\left(\alpha_{1, t}\right)$ & $0.1+0.7\left(\alpha_{2, t}\right)$ \\
\hline \hline Results & $\mathbf{W S P}_{1}$ & $\mathbf{W S P}_{2}$ \\
\hline NE SU price with $\zeta=0.25(\$)$ & 0.57 & 0.62 \\
\hline SUs served & 16 & 17 \\
\hline CP with $\zeta=0.25$ (\$) & 5.17 & 6.95 \\
\hline$\%$ rise in CP for $\zeta=0.25$ w.r.t. case 2 & 51.62 & 51.07 \\
\hline$\%$ rise in CP for $\zeta=0.25$ w.r.t. case 1 & 94.85 & 137.43 \\
\hline \hline
\end{tabular}




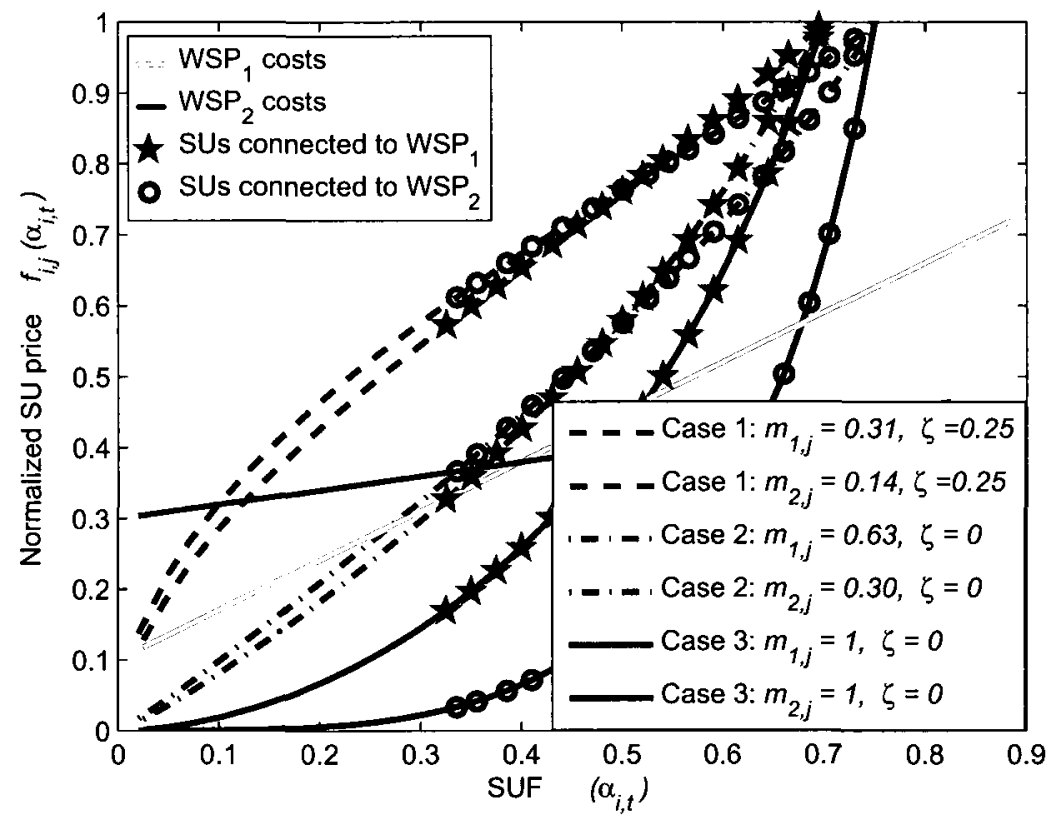

Figure 5.6: Scenario 2: SU price set by the two WSPs

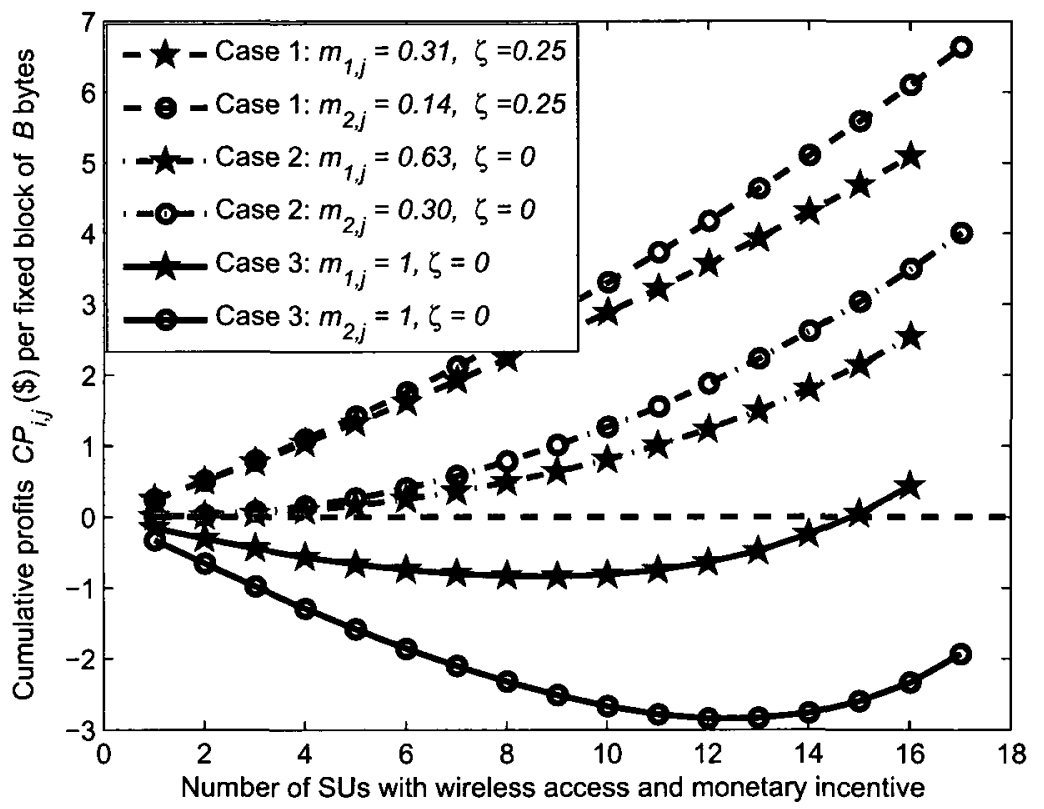

Figure 5.7: Scenario 2: CP obtained by the two WSPs from $\mathrm{SU}$ access 
Scenario 3: Dissimilar Average PU Demand and Symmetric WSP Costs

In this scenario, the PU demand at the $\mathrm{BS}$ of $\mathrm{WSP}_{2}$ is assumed to be significantly higher than the $\mathrm{PU}$ demand at $\mathrm{WSP}_{1}$, i.e., 0.22 and 0.48 respectively as shown in Table 5.5. But the costs borne by both the WSPs are assumed to be symmetric and given as $c_{i, j}=0.3+0.2\left(\alpha_{i, t}\right)$. It can be seen that, since the PU demand at the $\mathrm{BS}$ of $\mathrm{WSP}_{1}$ is lower than that at $\mathrm{BS}$ of $\mathrm{WSP}_{2}$, the initial SU price offered by $\mathrm{WSP}_{1}$ is slightly lower than that of $\mathrm{WSP}_{2}$, but the $\mathrm{SU}$ prices can still be observed to be competitive in spite of the difference in PU demand. Observing case 3 in Fig. 5.9 , it can be seen that the profit gained by $\mathrm{WSP}_{2}$ is significantly lower than $\mathrm{WSP}_{1}$, since fewer SUs could be accommodated at $\mathrm{WSP}_{2}$ due to the high $\mathrm{PU}$ demand in comparison to $\mathrm{WSP}_{1}$. However, in spite of the widely varying PU demand at the two BSs of the WSPs, the SU prices and the CPs seen from case 3 in Fig. 5.8 and Fig. 5.9 can be seen to follow a very competitive trend.

Table 5.5: Scenario 3: WSP parameters and results

\begin{tabular}{|c|c|c|}
\hline \hline Parameter & $\mathbf{W S P}_{1}$ & $\mathbf{W S P}_{2}$ \\
\hline PU demand $\alpha_{i, p u}$ & 0.22 & 0.48 \\
\hline Spectrum threshold $\alpha_{i, t h}$ & 0.9 & 0.85 \\
\hline Incentive cutoff limit $\alpha_{i, i c}$ & 0.7 & 0.75 \\
\hline Incentive cutoff factor $n_{i, j}$ & 1.83 & 3.66 \\
\hline WSP cost & $0.3+0.2\left(\alpha_{1, t}\right)$ & $0.3+0.2\left(\alpha_{2, t}\right)$ \\
\hline \hline Results & $\mathbf{W S P}_{1}$ & $\mathbf{W S P}_{2}$ \\
\hline NE SU price with $\zeta=0.25(\$)$ & 0.59 & 0.64 \\
\hline SUs served & 19 & 11 \\
\hline CP with $\zeta=0.25(\$)$ & 7.87 & 4.18 \\
\hline \% rise in CP for $\zeta=0.25$ w.r.t. case 2 & 37.65 & 45.79 \\
\hline$\%$ rise in CP for $\zeta=0.25$ w.r.t. case 1 & 96.97 & 106.17 \\
\hline \hline
\end{tabular}




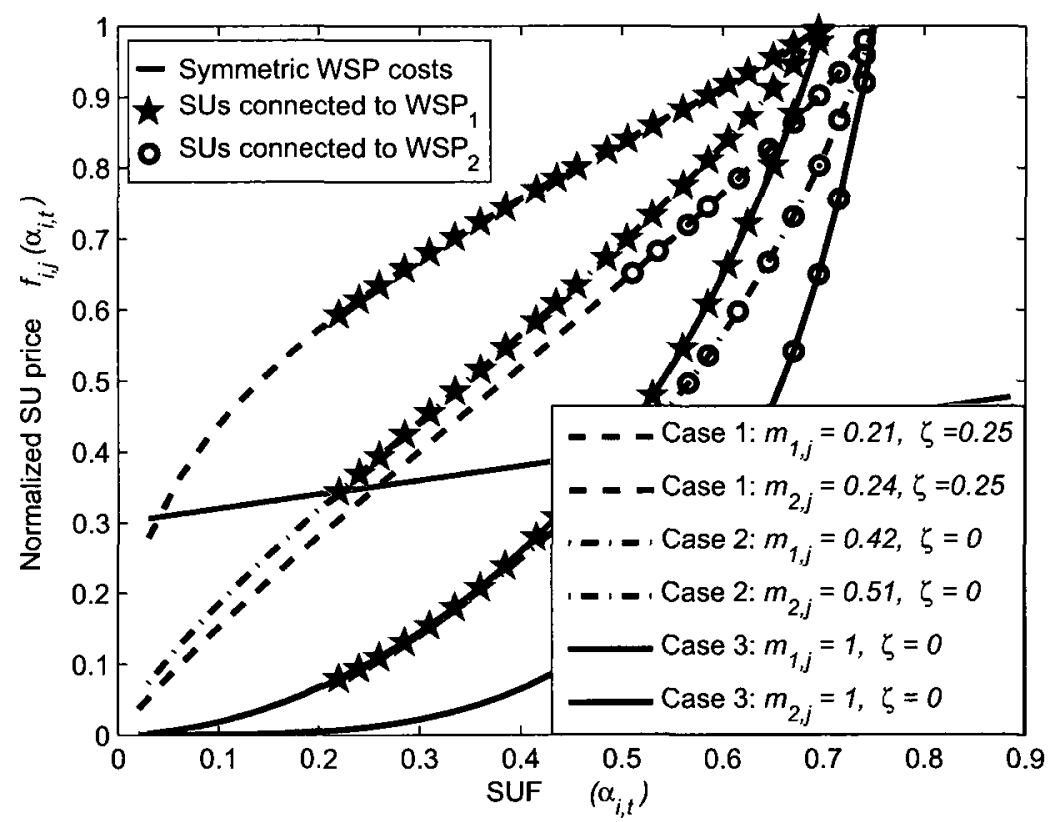

Figure 5.8: Scenario 3: SU price set by the two WSPs

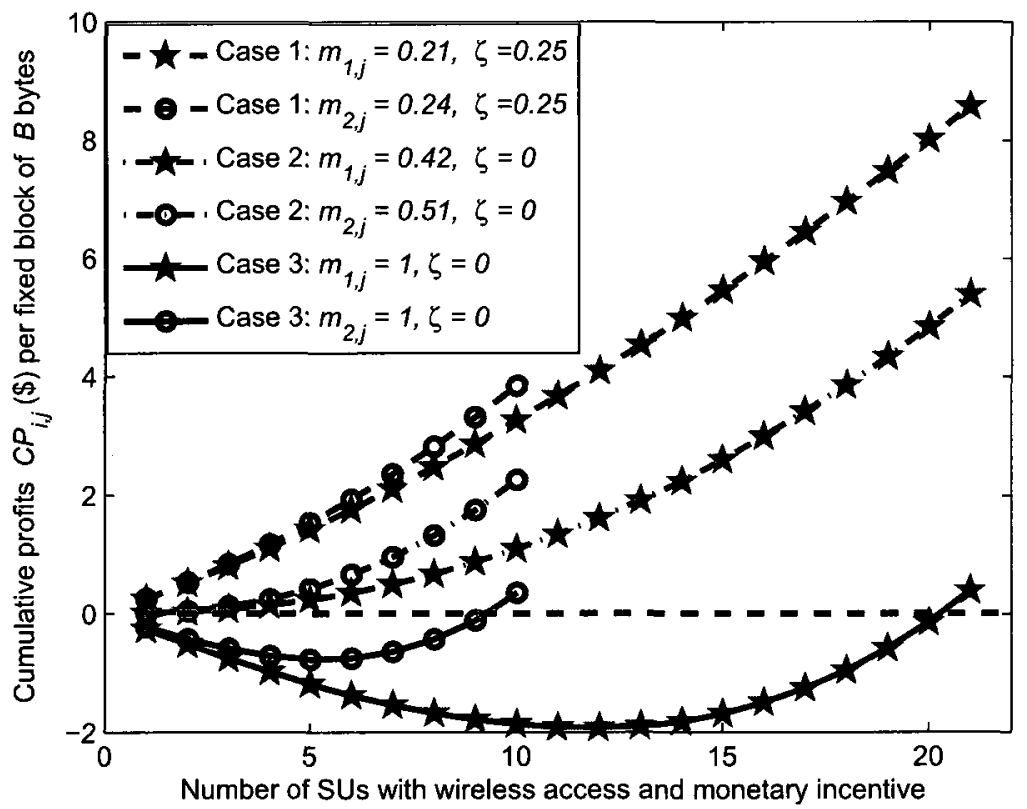

Figure 5.9: Scenario 3: CP obtained by the two WSPs from SU access 


\section{Scenario 4: Dissimilar Average PU Demand and Asymmetric WSP Costs}

This scenario could be considered as the most random scenario where the PU demand at the BSs of the two WSPs is widely dissimilar and so are the WSP cost as shown in Table 5.6. Due to the combined effect of the WSPs costs and the PU demand, which is significantly higher at $\mathrm{WSP}_{2}$ than at $\mathrm{WSP}_{1}$, the initial $\mathrm{SU}$ prices for $\mathrm{WSP}_{1}$ are lower and thus $\mathrm{WSP}_{1}$ attracts more $\mathrm{SUs}$ as seen in Fig. 5.10. It must be noted that, $\mathrm{WSP}_{2}$ cannot accommodate more $\mathrm{SU}$ since it is serving more number of PUs. However, the SU prices do show a competitive trend in spite of widely different PU demand and WSP costs. Figure 5.11 shows the CPs of the two WSPs and it can be seen that the two curves for case 3 are very close to each other with $\mathrm{WSP}_{2}$ showing higher initial CPs than $\mathrm{WSP}_{1}$. Thus even for the most extreme scenario, the SU prices and the WSP profits are observed to be highly competitive.

Table 5.6: Scenario 4: WSP parameters and results

\begin{tabular}{|c|c|c|}
\hline \hline Parameter & $\mathbf{W S P}_{1}$ & $\mathbf{W S P}_{2}$ \\
\hline PU demand $\alpha_{i, p u}$ & 0.22 & 0.52 \\
\hline Spectrum threshold $\alpha_{i, t h}$ & 0.9 & 0.85 \\
\hline Incentive cutoff limit $\alpha_{i, i c}$ & 0.7 & 0.75 \\
\hline Incentive cutoff factor $n_{i, j}$ & 1.83 & 3.66 \\
\hline WSP cost & $0.3+0.2\left(\alpha_{1, t}\right)$ & $0.1+0.7\left(\alpha_{2, t}\right)$ \\
\hline \hline Results & $\mathbf{W S P}_{1}$ & $\mathbf{W S P}_{2}$ \\
\hline NE SU price with $\zeta=0.24(\$)$ & 0.50 & 0.65 \\
\hline SUs served & 19 & 10 \\
\hline CP with $\zeta=0.24(\$)$ & 6.23 & 3.84 \\
\hline$\%$ rise in CP for $\zeta=0.24$ w.r.t. case 2 & 51.61 & 74.45 \\
\hline$\%$ rise in CP for $\zeta=0.24$ w.r.t. case 1 & 106.80 & 123.99 \\
\hline \hline
\end{tabular}




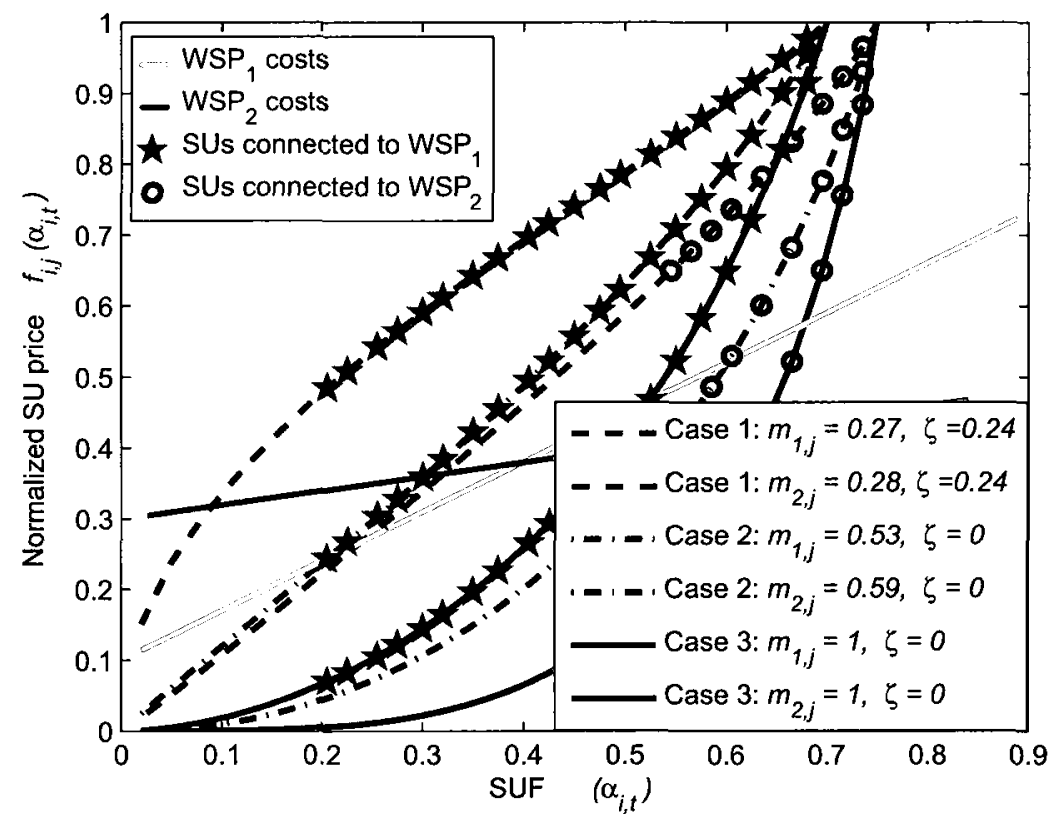

Figure 5.10: Scenario 4: SU price set by the two WSPs

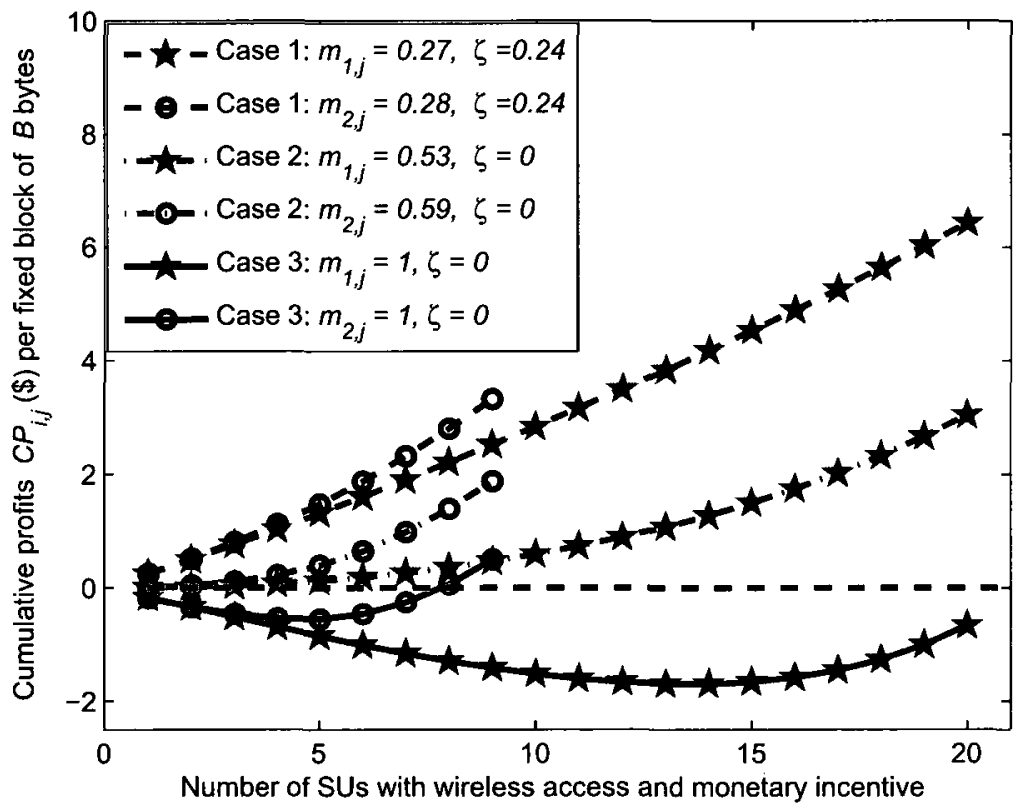

Figure 5.11: Scenario 4: CP obtained by the two WSPs from SU access 


\subsubsection{Effect of Assumptions on the WSP Profits}

The assumptions described in this chapter directly affect the WSP profits from SU access and a few key features relevant to this aspect are pointed out as follows:

- The additional profits to the WSPs from SU access shown previously consider the operations at only one BS. Therefore the total additional profits from SU access that could be obtained by the WSPs over a certain period of time $T_{u}$ will be a multiple of the number of BSs enabled with SU access controlled by the WSP.

- The WSP profits from SU access shown previously are based on the assumption that the SUs will join the BS if and only if they have a monetary incentive. However, in general this may not be the case, and the SUs with a monetary penalty may also acquire wireless access thus generating even higher profits from SU access than shown in this chapter.

- The selection of the configuration parameters also may have a significant impact on the WSP profits generated from SU access. The SUs are assumed to enter the network if and only if $\alpha_{i, t} \in\left(\alpha_{i, p u}, \alpha_{i, i c}\right)$. Therefore the prices charged to the SUs, which are controlled by the PLF, are restricted at one side by the static NE SU price $\left(S_{i, j}^{*}\right)$ and on the other by the PU price $\left(p_{i, j}\right)$. Therefore the SU service can be seen to be competitive priced, and profits achieved by the WSPs from SU service can also be observed to be competitive due to the differentiation of the SU wireless service based on $\zeta$.

- According to the assumptions and analysis in this chapter, it can be seen that the value of $\zeta$ is common to both the WSPs and can be calculated independently 
by each WSP based on the channel measurements.

- The WSPs do not need to observe and predict the prices set by other WSPs in the region before setting their own prices, since the dynamic SU price set by the WSP is based on PLF and (5.8), which only requires the WSP cost and the value of $\zeta$. Therefore, an independent WSP could provide SU access in an area by pricing the SUs using the dynamic pricing model and setting the appropriate PLF using (5.11),(5.8) and (5.14).

\subsection{Summary}

In this chapter equilibrium analysis has been presented which demonstrates the

potential for additional profits to the WSPs from SU access. The key points covered in this chapter are listed as follows:

- Equilibrium analysis based on differentiation of the SU wireless service in a WSP duopoly.

- Implementation of static equilibrium analysis on the dynamic pricing model.

- Competitive pricing among WSPs without cooperation among the WSPs.

- Mutual benefit of incentive pricing to the SUs and additional profits to the WSPs demonstrated based on the four scenarios considering varying PU demand and WSP cost.

- The willingness to pay of the SUs, the wireless environment, and the SUF at the BS are together considered in the final price the SUs' pay for temporary wireless access, which also guarantees profits to the WSPs. 
The conclusions, applications and directions for future work that can be drawn from this thesis are described in the final chapter. 


\section{Chapter 6}

\section{Conclusions, Applications and Future Work}

This chapter highlights the conclusions that can be drawn from this thesis in Section 6.1. The applications of the work presented in this thesis are given in Section 6.2, while suggested topics for future work are enlisted in Section 6.3.

\subsection{Conclusions}

A distributed framework for enabling dynamic SU access for the licensed spectrum in infrastructure based networks is described in this thesis. The distributed system framework proposed in this thesis allows an individual WSP to provide temporary wireless access directly to SUs, without the need for cooperation with other WSPs in the area and hence avoiding centralized coordinating entities. The proposed system framework can thus be considered as an intermediate step between current infrastructure based networks serving only PUs and the CR based infrastructure free networks of the future.

A comprehensive system perspective considering both the technological aspects and the economical aspects relevant to the proposed system framework has been considered in this thesis. 


\subsubsection{Technological Aspect}

The feasibility of proposed distributed framework allowing the orthogonal coexistence of PUs and SUs in the same spectrum band at the BS was shown in this thesis. Enabling DSA for SUs at the BS required a signaling framework for SU-BS interaction, which was also detailed in this thesis.

Since the SUs coexist with the PUs at the same BS, a mechanism for the differentiation of the SUs from the PUs based on a modification in the DSCP byte was proposed. A joint PU-SU RRM model at the BS was also proposed in this thesis, for enabling the BS to handle the orthogonal coexistence of SUs along with PUs at the BS without any deterioration to the PU service.

\subsubsection{Economic Aspect}

Since the potential implementation of any technology depends the economic viability, the economic aspects of pricing with equilibrium analysis have been considered in this thesis. Efficient spectrum utilization at the BS with DSA for SUs in infrastructure based networks required a pricing model that could attract SUs during periods of low PU demand. Therefore, a dynamic pricing model for pricing SUs based on the PU demand, spectrum utilization at the BS and PU price with an inherent property for SU admission control was proposed in this thesis. The pricing model thus offers discounted prices to the SUs, whenever the PU demand at the BS is low.

The potential additional profits that could be attained by the WSPs from SU access were shown by the implementation of equilibrium pricing analysis on the proposed incentive based pricing model. Competitive SU prices along with competitive profits to the individual WSPs were shown to be achieved in various scenarios, without any need for cooperation and coordination among the WSPs. 
The willingness to pay of the SUs, the wireless environment, and the SUF at the BS are together considered in the final price the SUs' pay for temporary wireless access, which also guarantees profits to the WSPs. The dual benefit of incentive based pricing for SUs as well as additional profits to the WSPs from SU access can be seen to be realized in this thesis.

\subsection{Applications}

In this section, potential applications of the distributed framework and the pricing model are described.

\subsubsection{Prepaid Customers in Current Infrastructure based Networks}

The proposed dynamic pricing model is based on a distributed framework and hence a single WSP can implement it without coordination with other WSPs in the area. Prepaid customers are currently charged higher prices in comparison to postpaid customers. However, there may be residual capacity at the BS available during off-peak hours. Using dynamic pricing, the prepaid customers could be attracted to gain access during these off-peak hours at lower prices. It must be noted that the prepaid customers are PUs and will need to be guaranteed QoS whenever they acquire wireless access. This could potentially be the first step for the WSPs towards enabling DSA based SU access.

\subsubsection{Dynamic Pricing for Generalized Commercial Applications}

The proposed dynamic pricing model offers a variety of tuning parameters for adjusting the prices. This pricing model is opportunistic with increasing price for subsequent users, where the price depends on the available capacity. The pricing 
model can also represents the price with respect to a baseline price, and thus provides a prospect of incentive to the users. These features can be very helpful for implementation in commercial applications, e.g., Airplane Ticketing, since such dynamic pricing inherently improves the utilization of the available capacity.

\subsection{Future Work}

Several aspects from the proposed work can be extended further. The following presents a list of suggestions for future work.

- A basic signaling framework is provided in this thesis, and more detailed work will be needed for moving this concept to the next stage, e.g., standardization.

- A joint PU-SU RRM model has been introduced in this thesis, and further scheduling algorithms for SUs with the appropriate throughput and fairness can be developed.

- A network selection criterion and a price based handoff scheme based on SU centric decisions have been introduced in this thesis. End-to-end simulation analysis can be performed for incorporating these algorithms.

- The pricing model and equilibrium analysis in this thesis have considered a single application class and a WSP duopoly. The results from this thesis can be extended to consider multiple application classes at the BS as well as more than two competing WSPs.

- The distributed framework and the pricing model introduced in this thesis could also provide applications to multi-hop relay networks. 


\section{References}

[1] M. A. McHenry, P. A. Tenhula, D. McCloskey, D. A. Roberson, and C. S. Hood, "Chicago spectrum occupancy measurements \& analysis and a long-term studies proposal," in TAPAS '06: Proceedings of the first international workshop on Technology and policy for accessing spectrum. New York, NY, USA: ACM, 2006, p. 1.

[2] Spectrum Policy Task Force, "Spectrum policy task force report," Federal Communications Commission, Tech. Rep. ET Docket No. 02-135, November 2002. [Online]. Available: http://www.fcc.gov/sptf/reports.html

[3] Q. Zhao and B. Sadler, "A survey of dynamic spectrum access," IEEE Signal Processing Magazine, vol. 24, no. 3, pp. 79-89, May 2007.

[4] I. Akyildiz, W.-Y. Lee, M. Vuran, and S. Mohanty, "A survey on spectrum management in cognitive radio networks," IEEE Communications Magazine, vol. 46, no. 4, pp. 40-48, April 2008.

[5] M. Buddhikot, "Understanding dynamic spectrum access: Models,taxonomy and challenges," in Proc. of IEEE DySPAN, April 2007, pp. 649-663.

[6] J. Mitola, III and G. Q. Maguire, Jr., "Cognitive radio: making software radios more personal," IEEE Personal Communications, vol. 6, no. 4, pp. 13-18, August 1999.

[7] S. Haykin, "Cognitive radio: brain-empowered wireless communications," IEEE Selected Areas in Communications, vol. 23, no. 2, pp. 201-220, February 2005.

[8] [Online]. Available: http://www.gsmarena.com/, website accessed: October 2009 - January 2010.

[9] J. M. Peha and S. Panichpapiboon, "Real-time secondary markets for spectrum," Telecommunications Policy, vol. 28, no. 7-8, pp. 603-618, August 2004.

[10] A. Attar, O. Holland, M. Nakhai, and A. Aghvami, "Interference-limited resource allocation for cognitive radio in orthogonal frequency-division multiplexing networks," IET Communications, vol. 2, no. 6, pp. 806-814, July 2008. 
[11] D. Niyato and E. Hossain, "Market-equilibrium, competitive, and cooperative pricing for spectrum sharing in cognitive radio networks: Analysis and comparison," IEEE Transactions on Wireless Communications, vol. 7, no. 11, pp. 4273-4283, November 2008.

[12] P. Lescuyer and T. Lucidarme, Evolved Packet System (EPS): The LTE and SAE Evolution of $3 G$ UMTS. Wiley, 2008.

[13] J. G. Andrews, A. Ghosh, and R. Muhamed, Fundamentals of WiMAX: Understanding Broadband Wireless Networking. Prentice Hall, 2007.

[14] J. Chapin and W. Lehr, "Cognitive radios for dynamic spectrum access - the path to market success for dynamic spectrum access technology," IEEE Communications Magazine, vol. 45, no. 5, pp. 96-103, May 2007.

[15] M. Marcus, "Real time spectrum markets and interruptible spectrum: new concepts of spectrum use enabled by cognitive radio," in Proc. of IEEE DySPAN, November 2005, pp. 512-517.

[16] T. Weiss and F. Jondral, "Spectrum pooling: an innovative strategy for the enhancement of spectrum efficiency," IEEE Communications Magazine, vol. 42, no. 3, pp. S8-14, March 2004.

[17] M. Buddhikot, P. Kolodzy, S. Miller, K. Ryan, and J. Evans, "Dimsumnet: new directions in wireless networking using coordinated dynamic spectrum," in Proc. of WoWMoM, June 2005, pp. 78-85.

[18] J. Perez-Romero, O. Salient, R. Agusti, and L. Giupponi, "A novel on-demand cognitive pilot channel enabling dynamic spectrum allocation," in Proc. of IEEE DySPAN, April 2007, pp. 46-54.

[19] O. Ileri, D. Samardzija, and N. Mandayam, "Demand responsive pricing and competitive spectrum allocation via a spectrum server," in Proc. of IEEE DySPAN, November 2005, pp. 194-202.

[20] J. Acharya and R. Yates, "Resource and power costs in dynamic spectrum allocation," in Proc. of CISS, March 2008, pp. 938-943.

[21] P. Cordier, P. Houze, S. B. Jemaa, O. Simon, D. Bourse, D. Grandblaise, K. Moessner, J. Luo, C. Kloeck, K. Tsagkaris, R. Agusti, N. Olaziregi, Z. Boufidis, E. Buracchini, P. Goria, and A. Trogolo, "E2R cognitive pilot channel concept," in 15th IST Mobile and Wireless Communications Summit, June 2006. 
[22] Q. Zhao, L. Tong, A. Swami, and Y. Chen, "Decentralized cognitive MAC for opportunistic spectrum access in Ad Hoc networks: A POMDP framework," IEEE Selected Areas in Communications, vol. 25, no. 3, pp. 589-600, April 2007.

[23] J. Comm and K. Burge, Twitter Power: How to Dominate Your Market One Tweet at a Time. John Wiley \& Sons, 2009.

[24] S. Vegesna, IP Quality of Service. Cisco Press, 2001.

[25] H. Al-Zubaidy, I. Lambadaris, and I. Viniotis, "Optimal resource scheduling in wireless multiservice systems with random channel connectivity," in Proc. of IEEE Globecomm, December 2009.

[26] X. Liu, E. K. P. Chong, and N. B. Shroff, "A framework for opportunistic scheduling in wireless networks," Computer Networks, vol. 41, no. 4, pp. 451 474, 2003.

[27] J. Perez-Romero, O. Sallent, and R. Agusti, "A novel metric for context-aware RAT selection in wireless multi-access systems," in Proc. of IEEE ICC, June 2007, pp. 5622-5627.

[28] O. Sallent, J. Perez-Romero, R. Ljung, P. Karlsson, and A. Barbaresi, "Operator's RAT selection policies based on the fittingness factor concept," in 16th IST Mobile and Wireless Communications Summit, July 2007, pp. 1-5.

[29] F. Bari and V. C. Leung, "Automated network selection in a heterogeneous wireless network environment," IEEE Network Magazine, vol. 21, no. 1, pp. 34-40, Jan.-Feb. 2007.

[30] A. Goldsmith and S.-G. Chua, "Variable-rate variable-power MQAM for fading channels," IEEE Transactions on Communications, vol. 45 , no. 10, pp. 12181230 , Oct 1997.

[31] S. Boyd and L. Vandenberghe, Convex Optimization. Cambridge, U.K: Cambridge University Press, 2004.

[32] R. Barnes, Ecomonic Analysis, An Introduction. London, U.K: Butterworth \& Co. Ltd., 1971.

[33] B. Polak, Game Theory Lecture Notes. Yale University open courses, 2007. [Online]. Available: http://oyc.yale.edu/economics/game-theory/contents/sessions.html

[34] P. K. Dutta, Strategies and Games: Theory and Practice. The MIT Press, 1999. 
[35] R. S. Khemani and D. M. Shapiro, Glossary of Industrial Organization Economics and Competition Law. Directorate for Financial, Fiscal and Enterprise Affairs, Organization for Economic Co-operation and Development (OECD), 1993.

[36] D. Niyato and E. Hossain, "Microeconomic models for dynamic spectrum management in cognitive radio networks," in Cognitive Wireless Communication Networks, E. Hossain and V. Bhargava, Eds. Springer US, 2007, pp. 391-423.

[37] 3GPP TR 36.942 v1.2.0, "E-UTRA Radio Frequency (RF) system scenarios (Release 8)," June 2007. 


\section{Appendix A}

\section{Microeconomics}

Economics is the social science that studies the production, distribution and consumption of products and services, and is primarily based on demand for a particular product and the supply for that product. Economics can be classified into two main branches:

Microeconomics: This branch primarily deals with the finances for a particular individual or a firm based on demand and supply of a product.

Macroeconomics: This branch has a much wider perspective, which considers the finances of the entire nation based on labor, resources, industrial development and trade. In this thesis, basic principles of microeconomics have that have been used are given in this appendix.

\section{A.1 Demand Curve}

The law of demand states that, considering all other factors remaining constant, the price for a product is inversely proportional to the quantity demanded, i.e. higher the price for a product, less is the demand for that product. For example, since the price of gold is high, the demand for gold is low, while the price of rice is low, and demand for rice is high. It must be noted that, the final market price of a product 


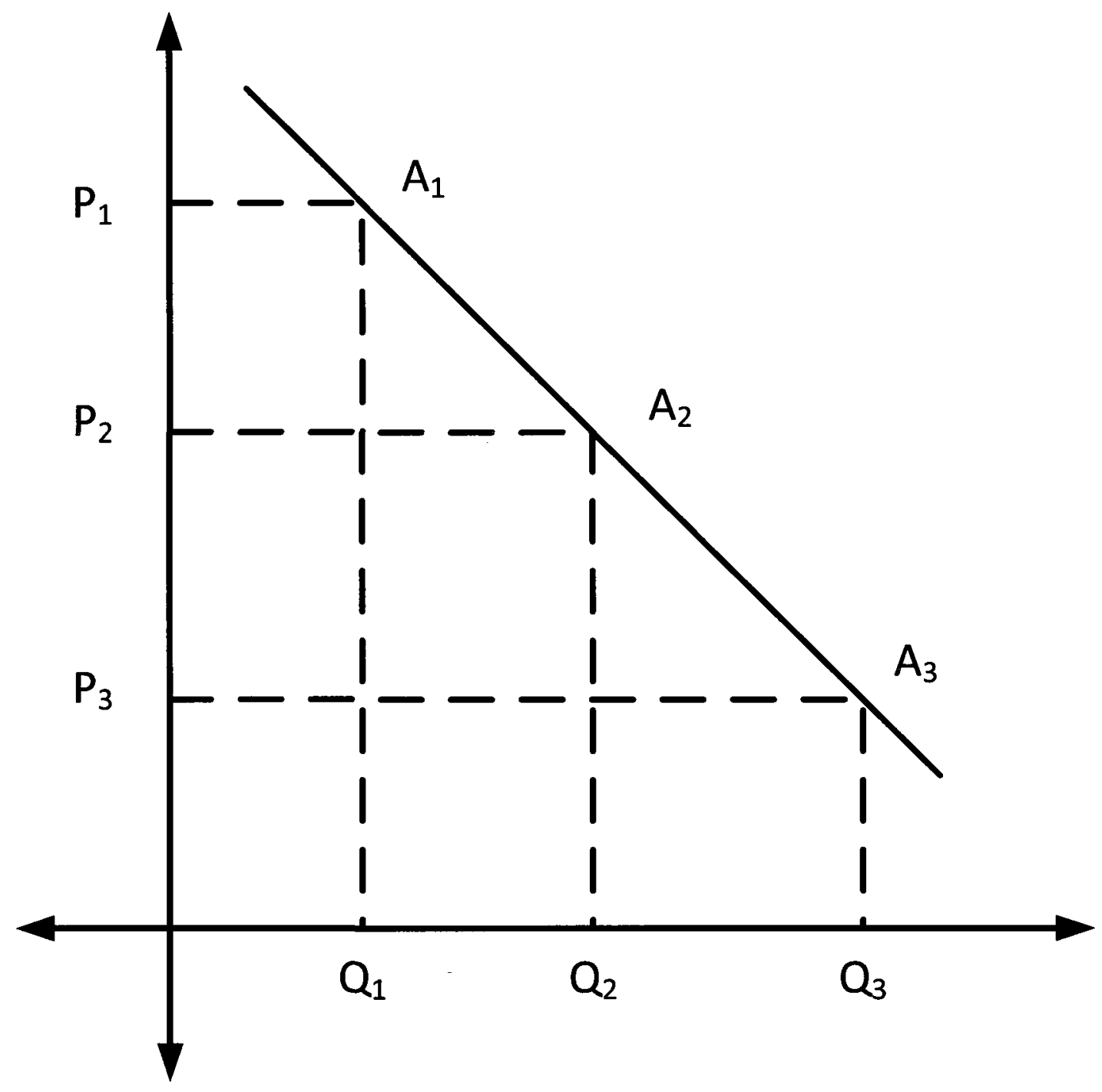

Figure A.1: Demand Curve

also depends upon the supply of the product that can be provided to the market. The demand for a particular product is represented by a downward sloping curve, where the price of the product is plotted with respect to the quantity demanded as illustrated in Fig. A.1. Considering $P_{k}$ as the price charged for a product by a firm $k$ for a quantity demanded $Q_{k}$, each point on the demand curve for the product provides 
a correlation between this price charged and quantity demanded. The movement along the points $A_{1}$ to $A_{2}$ to $A_{3}$ on the demand curve provides different prices for a different quantities demanded, in accordance with the demand curve.

\section{A.2 Microeconomics Models}

This section provides an overview of the three forms of markets in microeconomics, and introduces the notion of product differentiation.

\section{A.2.1 Forms of Economic Markets and Oligopoly}

Microeconomic theory defines three forms of the product market based on the number of firms selling a particular product, which can be listed as follows [32]:

1. Monopoly $P_{k}(M)$ : Single firm

2. Perfect Competition $P_{k}(C)$ : Large number of firms

3. Oligopoly $P_{k}(O)$ : Few firms with a large market share

The product prices in the respective forms of the product market are denoted by $P_{k}($ form $)$. With the buyers or consumers of this product considered to be large in number, this product price $P_{k}$ set by a firm ${ }_{k}$ for the same homogeneous product thus depends upon the form of the product market involved [32].

Considering a single firm selling a particular product in the market, the product price $P_{k}(M)$ is the highest possible price the firm ${ }_{k}$ could charge for a product based on the demand curve, thus assuring maximum profits. On the other hand, if a large number of firms are selling the same homogeneous product, the product prices $P_{k}(C)$ 
will decrease to the marginal costs with individual firm profits being negligible, due to intense competition for selling the same product [32].

However, in an oligopolistic market, only a few firms are involved in the product market. The firms in an oligopolistic market need to observe the behavior of their competing firms with respect to price or quantity produced, before setting the price [32]. Thus game theoretic analysis is a useful tool in analyzing the strategic effects based on price or quantity based imperfect competition among firms in oligopoly $[33,34]$. A comparison of the product prices for the same product in the three forms of the market is given as follows [32]:

$$
P_{k}(C) \leq P_{k}(O) \leq P_{k}(M)
$$

The wireless service market can thus be considered as an oligopolistic market, since there are only a few WSPs providing wireless service in any region. In the context of this thesis, the firms are the WSPs, the consumers or buyers are the SUs, while the product being sold is the wireless service.

\section{A.2.2 Imperfect Competition based on Prices}

In oligopoly, since there are limited number of competing firms, the competition is imperfect. The firms in an oligopoly can compete on the basis of the following two aspects:

1. Quantity based competition (Cournot model)

2. Price based competition (Bertrand model)

It can be proved that when firms select quantities for competing against each other, the NE price obtained lies between the monopoly price and the perfect competition 
price as given in (A.1). However, when firms compete based on prices, the NE price equals to the marginal costs of the firms and this leads to zero profits to the firms [33,34]. An intuitive proof for price based competition among competing firms in an oligopoly taken from [33] is presented below.

Two firms are assumed to sell an identical product in the market, and the marginal cost incurred is $C$. These firms compete on prices and set the prices $P_{1}$ and $P_{2}$ respectively, such that $0 \leq P_{k} \leq 1$ and the prices are set simultaneously. The demand curve is assumed to be given as $Q(P)=1-P$, where $P$ is the lower of the two prices. Therefore the demand for firm ${ }_{1}$ can be given as

$$
Q_{1}= \begin{cases}1-P_{1}, & \text { if } P_{1}<P_{2}, \\ 0, & \text { if } P_{1}>P_{2}, \\ 1-\frac{P_{1}}{2} & \text { if } P_{1}=P_{2} .\end{cases}
$$

The payoffs for the firms can be given as

$$
Q_{1} \times P_{1}-Q_{1} \times C=Q_{1}\left(P_{1}-C\right) .
$$

Thus the best responses for firm 1 based on the different prices set by firm 2 can be given as follows:

$$
B R_{1}\left(P_{2}\right)= \begin{cases}P_{1}>P_{2}, & \text { if } P_{2}<C, \\ P_{1}=P_{2}-\epsilon, & \text { if } P_{2}>C, P(M) \geq P_{2}, \\ P_{1}=P(M) & \text { if } P_{2}>P(M), \\ P_{1} \geq C=P_{2} & \text { if } P_{2}=C,\end{cases}
$$

where $\mathrm{P}(\mathrm{M})$ is the monopoly price for the product and $\epsilon$ is a very small positive number between 0 and 1, e.g., $\epsilon=0.002$. 
Thus it can be observed that the NE price is $\left(P_{1}=C, P_{2}=C\right)$, since if any price is set higher $C$, the best response price by either firm will bring the price back to NE price in steps of $\epsilon$. Thus it can be seen that for two firms competing in prices, the $\mathrm{NE}$ price will be equal to the costs leading to zero profits.

\section{A.2.3 Differentiation of Products}

In practice, such a scenario with firms earning zero profits never occurs, since the firms differentiate their products based on quality, brand name, advertising, target consumers or buyers, etc. This differentiation in products allows the firms to reduce the competition with other firms, and thus the firms stand to generate higher profits. Therefore, product differentiation allows to firms in an oligopoly to tend towards monopoly, since the products of the competing firms in the same product market are no longer homogeneous. 